\title{
Considerations in designing an adult hearing screening programme
}

Citation for published version (APA):

Linssen, A. M. (2015). Considerations in designing an adult hearing screening programme. [Doctoral Thesis, Maastricht University]. Uitgeverij BOXPress. https://doi.org/10.26481/dis.20150213al

Document status and date:

Published: 01/01/2015

DOI:

10.26481/dis.20150213al

Document Version:

Publisher's PDF, also known as Version of record

\section{Please check the document version of this publication:}

- A submitted manuscript is the version of the article upon submission and before peer-review. There can be important differences between the submitted version and the official published version of record.

People interested in the research are advised to contact the author for the final version of the publication, or visit the DOI to the publisher's website.

- The final author version and the galley proof are versions of the publication after peer review.

- The final published version features the final layout of the paper including the volume, issue and page numbers.

Link to publication

\footnotetext{
General rights rights.

- You may freely distribute the URL identifying the publication in the public portal. please follow below link for the End User Agreement:

www.umlib.nl/taverne-license

Take down policy

If you believe that this document breaches copyright please contact us at:

repository@maastrichtuniversity.nl

providing details and we will investigate your claim.
}

Copyright and moral rights for the publications made accessible in the public portal are retained by the authors and/or other copyright owners and it is a condition of accessing publications that users recognise and abide by the legal requirements associated with these

- Users may download and print one copy of any publication from the public portal for the purpose of private study or research.

- You may not further distribute the material or use it for any profit-making activity or commercial gain

If the publication is distributed under the terms of Article $25 \mathrm{fa}$ of the Dutch Copyright Act, indicated by the "Taverne" license above, 
Considerations in designing an adult hearing screening programme 
(C) Anouk Linssen, Maastricht 2015

ISBN: 978-94-6295-066-5

Cover design by Anouk Linssen \& Proefschriftenmaken.nl || Uitgeverij BOXPress Printed by Proefschriftenmaken.nl || Uitgeverij BOXPress

Published by Uitgeverij BOXPress, 's Hertogenbosch 


\title{
Considerations in designing an adult hearing screening programme
}

\author{
Proefschrift \\ ter verkrijging van de graad van doctor \\ aan de Universiteit Maastricht, \\ op gezag van de Rector Magnificus, Prof. dr. L.L.G. Soete, \\ volgens het besluit van het College van Decanen, \\ in het openbaar te verdedigen \\ op vrijdag 13 februari 2015 om 12.00 uur
}

door

Anouk Maria Linssen 


\section{Promotor}

Prof. dr. B. Kremer

\section{Copromotoren}

Dr. L.J.C. Anteunis

Dr. M.A. Joore

\section{Beoordelingscommissie}

Prof. dr. R.J. Stokroos (voorzitter)

Prof. dr. G.J. Dinant

Prof. dr. S.M.A.A. Evers

Dr. J.P.C. Grutters (Radboudumc Nijmegen)

Prof. dr. ir. A.F.M. Snik (Radboudumc Nijmegen)

The research described in this thesis was performed at the School for Mental Health and Neuroscience of the Faculty of Health, Medicine and Life Sciences of Maastricht University and the Department of Otorhinolaryngology, Head and Neck Surgery of the Maastricht University Medical Centre. The research was partially funded by the Heinsius Houbolt Foundation. 


\section{Contents}

Chapter 1

General introduction

7

Chapter 2

Being labelled as hearing-impaired based on an objective hearing screening test: reactions of adults without hearing complaints

\section{Chapter 3}

Qualitative interviews on the beliefs and feelings of adults towards their ownership, but non-use of hearing aids

Chapter 4

The effects and costs of a hearing screening and rehabilitation programme

in residential care homes for the elderly in the Netherlands

Chapter 5

Predictors of hearing acuity: cross-sectional and longitudinal analysis 59

Chapter 6

The cost-effectiveness of different hearing screening strategies for 50 - to

70-year-old adults: a Markov model

Chapter 7

General discussion

Chapter 8

Valorisation

Bibliography

Summary

Samenvatting 153

About the author 159

Dankwoord 163 



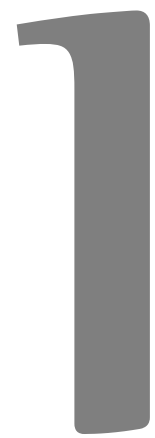

General introduction 



\section{Rationale for adult hearing screening}

According to the World Health Organisation, someone is hearing-impaired when the unaided pure-tone audiometric hearing threshold is greater than $25 \mathrm{~dB}$ in the better ear, averaged across $0.5,1,2$, and $4 \mathrm{kHz}$ (World Health Organisation, 2013). The prevalence of hearing impairment increases with age, ranging from approximately $11 \%$ in adults aged $55-59$ years to $88 \%$ in adults aged $\geq 85$ years (Kiely et al., 2012a).

Untreated hearing loss has a variety of negative consequences, including social isolation, loneliness, psychosocial distress, anxiety, and depression (Gopinath et al., 2009; Monzani et al., 2008; Nachtegaal et al., 2009). As hearing loss affects communication, it also decreases the quality of life of the spouse, other relatives, and close friends of the person with hearing loss (Scarinci et al., 2008; Wallhagen et al., 2004).

Hearing aid fitting forms the conventional rehabilitation option, although several alternatives are available as well. Hearing aid fitting can increase the quality of life of people with a hearing loss (Chia et al., 2007; Chisolm et al., 2007; Vuorialho et al., 2006). It is a cost-effective intervention (Chao \& Chen, 2008; Joore et al., 2003a). Depending on the type of hearing aid, between $89 \%$ and $100 \%$ of people find hearing aids acceptable (Davis et al., 2007). Nevertheless, help-seeking and hearing aid uptake among adults with hearing loss is low. Duijvestijn and colleagues (2003), for example, found that although $56 \%$ of the hearing-impaired adults (aged $\geq 55$ years) had sought professional help, only $45 \%$ of these help-seekers took up hearing aids.

Adults with hearing loss generally postpone seeking help until five to ten years after the onset of their hearing loss (Davis et al., 2007; Laplante-Lévesque et al., 2012a; Leegwater \& Lammerts van Bueren, 2005; Meister et al., 2008). The main reasons for the delay in help-seeking is unawareness or denial of the hearing loss and a belief that one can still manage without hearing aids (Van Thiel, 2010).

Hearing loss is objectively measurable before an affected individual becomes aware of it. In the period between the onset of the hearing loss and help-seeking, the hearing loss can already negatively affect his or her well-being and the well-being of his or her significant others. Timely help-seeking and early rehabilitation is important because adults who start using hearing aids early - that is, at a relatively young age or when their hearing loss is still relatively mild - do not only have more years with benefit, but also have more benefit from their hearing aids during later life than adults who started using hearing aids late (Davis et al., 2007). This can be explained by the fact that older adults have more difficulty adapting to hearing aids and learning to use new technology because of poorer cognitive performance and poorer learning ability (Humes, 2007; Janacsek et al., 2012). Poorer cognitive performance is also associated with a difficulty formulating needs during a hearing aid trial, resulting in a suboptimal hearing aid fit (Lunner, 2003). Besides, older adults have poorer manual dexterity, which causes greater difficulty in handling hearing aids and less benefit (Kumar et al., 2000).

Adult hearing screening has been proposed as a potential strategy to motivate adults to seek help earlier (Davis et al., 2007; Thodi et al., 2013; Yueh et al., 2003). The 
objective of this thesis is to gain more insight into how to design an adult hearing screening programme for nationwide implementation.

\section{Criteria for screening programmes}

To be eligible for nationwide implementation, a screening programme should meet several universal criteria. The most frequently used criteria are those of the World Health Organisation formulated by Wilson and Jungner in 1968 and supplemented by Andermann and colleagues in 2008 (Table 1). The criteria relate to four topics: the rationale for screening, the benefits and harms of screening, the screening tests, and organisational aspects.

Table 1. World Health Organisation criteria for screening

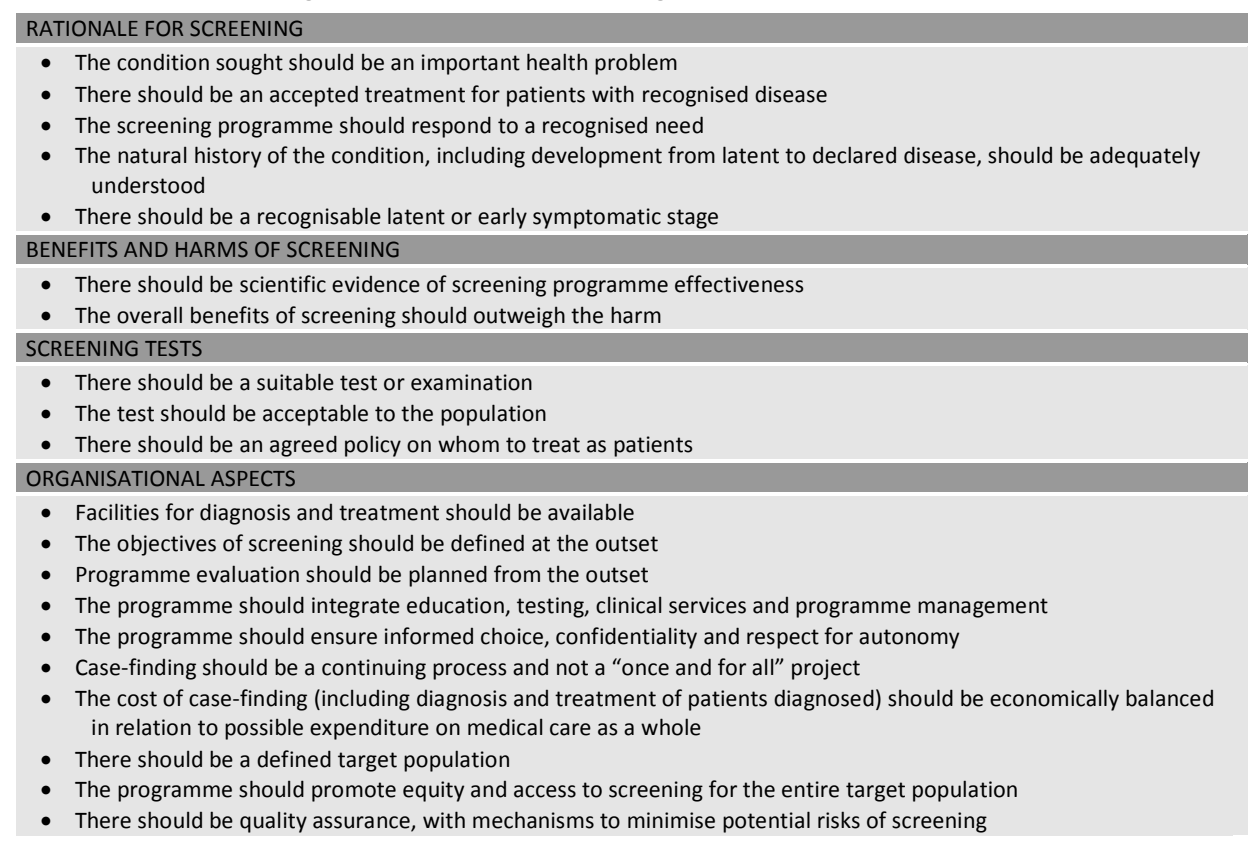

\section{Rationale for screening}

The condition sought should be an important health problem, there should be an accepted treatment for patients with recognised disease, the screening programme should respond to a recognised need, the natural history of the condition should be adequately understood, and there should be a recognisable latent or early symptomatic stage. As outlined at the beginning of this chapter, untreated hearing loss is an important health problem because of the large number of people involved and the negative consequences for affected people and their significant others. Although hearing loss cannot be cured, acceptable, effective, and cost-effective rehabilitation 
options (i.e. treatments for the consequences of hearing loss) are available (Chao \& Chen, 2008; Chia et al., 2007; Chisolm et al., 2007; Davis et al., 2007; Joore et al., 2003a; Vuorialho et al., 2006). The fact that hearing-impaired adults do not seek help or wait several years before seeking help supports the need for screening to underline the importance of good hearing, to make people earlier aware of their decreased hearing acuity, and to stimulate help-seeking. Hearing deterioration is a normal part of the aging process, generally starting at an age of 40 to 50 years and slowly progressing during later life. Hearing loss is objectively detectable at an early (latent) stage. Thus, the criteria regarding the rationale for screening are met.

\section{Benefits and harms of screening}

There should be scientific evidence of screening programme effectiveness and the overall benefits of screening should outweigh the harm. Studies in the United Kingdom showed that screening can triple hearing aid ownership among middle-aged adults (Davis et al., 1992; Stephens et al., 1990). Additionally, the only randomised controlled trial to the effectiveness of screening so far showed that screening with a hand-held audiometric device can almost double the 1-year incidence of hearing aid use (Yueh et al., 2010). These studies indicate that screening is effective indeed. The potential harms of screening have not been studied yet. It is possible that screening causes harmful psychosocial effects as a result of labelling.

\section{Screening tests}

There should be a suitable and acceptable screening test and an agreed policy on whom to treat as patients. Adult hearing screening tests meet these criteria. Many hearing screening tests are available nowadays, including the tune fork test, whispered voice test, speech-in-noise telephone test, speech-in-noise internet test, screening questionnaires, and hand-held audiometric devices. The tests are reasonably accurate although for some tests agreement lacks on whom to label as hearing-impaired (see reviews by Chou et al. (2011) and Bagai et al. (2006)). The acceptability of the tests is high (Davis et al., 2007; Koopman et al., 2008).

\section{Organisational aspects}

Criteria regarding organisational aspects concern feasibility, accessibility, target groups, confidentiality, respect for autonomy, continuity, quality assurance, costs, and programme evaluation (Table 1). A national adult hearing screening programme for Dutch citizens has been considered but not implemented yet due to a lack of information on some organisational aspects. The Advisory Council on Health Research concluded that more information is needed on the cost benefit ratio and organisational strategies to optimise this ratio (Raad voor Gezondheidsonderzoek, 2003). More specific, they recommended research to the cost-effectiveness of screening, the right time for screening, the best target population, the acceptability of screening, the 
willingness to participate, strategies to improve participation, and factors that limit the effectiveness.

\section{Objective and research questions}

The objective of this thesis is to gain more insight into how to design an adult hearing screening programme for nationwide implementation. The research questions are:

1. Does screening cause harm and how can potential harms be prevented or minimised?

2. What should the screening programme include to prevent non-use of dispensed hearing aids?

3. What is the best target population?

4. What is the best screen instrument?

\section{Outline of the thesis}

The overall benefits of a screening programme should outweigh the harms and the programme should include quality assurance with mechanisms to minimise these harms (Andermann et al., 2008). To identify potential harms of screening and clues for prevention or minimisation of these harms, we performed a qualitative study on the reactions of adults without hearing complaints to being labelled as hearing-impaired based on an objective hearing screening test. The specific population was selected because we expected that, if screening would have a harmful effect, it would most likely become apparent in adults who unexpectedly screened positive for hearing impairment. This study is described in Chapter 2. The screening test used was the Klangtester V3 Terminal, developed by Klangspektrum $\mathrm{GmbH}$, that was adapted to present the screen outcome as an 'ear age'. The value of the ear age and the suitability of the adapted Klangtester as screen instrument is discussed in Chapter 2 as well.

Another requirement a national screening programme should fulfil is that there should be an acceptable treatment (Wilson \& Jungner, 1968). Hearing aid fitting, the conventional rehabilitation option for hearing impairment, is generally considered acceptable (Davis et al., 2007). Nevertheless, up to a quarter of the people who own hearing aids never use them (Bertoli et al., 2009; Hartley et al., 2010; Kochkin, 2010). In current practice, people seek help for their hearing loss and take up hearing aids when they perceive a need for rehabilitation. In a screening setting, people who take up hearing aids will in general have less severe hearing complaints and perceive less of a need for rehabilitation. Consequently, the likelihood of non-use might be even higher. Non-use of dispensed hearing aids is undesirable for the health benefits missed as well as for the inefficient use of financial resources. Preventing non-use will improve the cost benefit ratio of screening. Chapter 3 reports on a qualitative study that aimed to 
increase our understanding of adults who own but not use hearing aids in order to get clues for non-use prevention.

The subsequent three chapters in this thesis address the selection of the target population. The target population should be clearly defined before a screening programme is considered for nationwide implementation (Andermann et al., 2008). Chapter 4 describes the effects and costs of a hearing screening and rehabilitation programme that was organised in eight residential care homes for the elderly in the Netherlands. We hypothesised that easy accessibility of hearing care professionals would be a great facilitator for help-seeking and hearing aid uptake. Based on the effects and costs, we discuss whether or not people living in care homes for the elderly form a suitable target for screening. Chapter 5 reports on an observational study to predictors of hearing acuity. Cross-sectional as well as longitudinal analysis were performed. The study aimed to give insight into the value of high-risk group screening with target selection based on the presence of comorbidities and on demographic, anthropometric, and life style factors. Chapter 6 presents a state transition model used to assess the optimal target age for screening based on analysis of the cost-effectiveness of several screening strategies.

The state transition model from Chapter 6 was also used to determine what type of screening (screen instrument) would be best. We analysed and compared the costs and effects of the following four types of screening: telephone screening, internet screening, screening at the practice of the general practitioner (GP) with a hand-held screening device, and screening at the GP practice with a standard audiometer.

Chapter 7 gives a summary of the main findings with regard to the research questions, followed by a discussion about methodological issues. The chapter ends with a statement of our conclusions and recommendations. Finally, Chapter 8 addresses valorisation. 



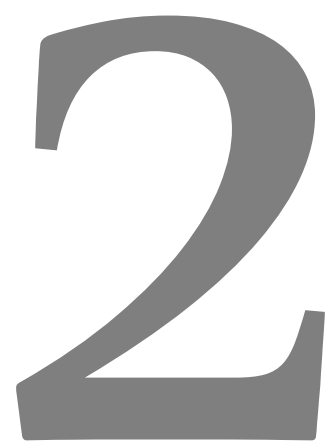

Being labelled as hearing-impaired based on an objective hearing screening test: reactions of adults without hearing complaints

Anouk M Linssen Lucien JC Anteunis Manuela A Joore 


\begin{abstract}
This qualitative study explored adults' reactions to being labelled as hearing-impaired based on an objective hearing screening test. Individual face-to-face, semi-structured interviews were conducted with seven adults (aged 52-79 years) without hearing complaints who screened positive for a hearing impairment. Thematic analysis of the interviews showed that the positive screen outcome prompted the participants to reflect on the test validity, their perceived hearing ability, their risk for hearing loss, their perceived need for professional help, and their willingness to start using hearing aids. The outcome did not stimulate them to start using hearing aids. However, it caused surprise, disbelief, incomprehension, disappointment, concern, emotional pain, and sadness, which are emotions known to be associated with an increasing awareness of hearing loss. This indicates that objective hearing screening can incite or accelerate the hearing loss recognition process and might indirectly motivate people to seek help earlier. A disadvantage of objective screening was that it could cause feelings of indignation (indicator of harm) but the results suggest that this can probably be overcome by using a highly accurate objective test under optimal testing conditions.
\end{abstract}




\section{Introduction}

Hearing loss forms a threat for general public health because it affects many people and can have negative consequences when left untreated (Arlinger, 2003). Age-related hearing loss cannot yet be cured, but hearing complaints can be reduced effectively by the use of hearing aids (Chisolm et al., 2007; Laplante-Lévesque et al., 2012a; Vuorialho et al., 2006). Nevertheless, adults generally postpone seeking help until five to ten years after the onset of their hearing loss (Davis et al., 2007; Laplante-Lévesque et al., 2012a; Leegwater \& Lammerts van Bueren, 2005; Meister et al., 2008). People mainly postpone seeking help because they are unaware or in denial of the hearing loss and believe they can still manage without hearing aids (Van Thiel, 2010).

Programmes for adult hearing screening are an effective way to improve hearing aid uptake (Davis et al., 1992; Stephens et al., 1990; Yueh et al., 2010). In order to implement large-scale testing, screening programmes should meet several criteria (Andermann et al., 2008; Wilson \& Jungner, 1968). One important criterion, besides improving health outcomes, is that the benefit of the screening programme should outweigh the physical and emotional harm caused by the screening test. Literature reviews that have investigated whether adult hearing screening meets these criteria conclude that the potential harm of adult hearing screening has never been evaluated in randomised trials or controlled observational studies (Baguley, 2008; Chou et al., 2011; Mulrow \& Lichtenstein, 1991). There is one qualitative focus group study that examined people's experience of participation in a hearing screening programme (Davis et al., 2007). Although the participants felt the after-care could have been better organised, they were enthusiastic about the screening itself and did not report any harm due to the screening. All of the participants in the focus group started using hearing aids following the screening and felt that the hearing aids improved their lives, which might explain why none of them reported harm or were negative about the screening programme. Furthermore, the participants may have forgotten the shortterm harm associated with the screening, because the focus group study took place two years after the test.

To our knowledge, no existing studies have examined the short-term impact of hearing screening on adults without hearing complaints. However, literature is available about the short-term reactions that people have to being diagnosed with a hearing impairment based on a standard clinical diagnostic tests. Martin and colleagues (1989) conducted a study in which they asked participants to indicate in a list of emotions how they felt during the hearing evaluation and found that the initial emotional reactions to the diagnosis included (from most to least frequently) sadness, worry, fear, disappointment, anger, surprise, shock, and no reaction at all. Some female participants also reported feelings of embarrassment, depression, relief, and anxiety. In addition, Weinberger (1980) found that being officially diagnosed with a hearing impairment significantly lowered participants' self-esteem, satisfaction with work, and satisfaction with relationships with significant others (all assessed two weeks after the diagnosis). However, when assessed two months after the diagnosis, those measures had returned to the same level as before diagnosis. An important difference between 
people who request a full diagnostic hearing test, like Martin's and Weinberger's study participants, and people who participate in a screening programme is that the former generally suspect they have a hearing loss, while the latter often do not. Consequently, their reactions to a positive test outcome might differ.

This qualitative study aimed to explore the short-term impact of a positive hearing screen outcome. We were particularly interested in the potential harm of adult hearing screening and believed that, if a positive screening test outcome caused harm, this would be most apparent in people who had been unaware of the hearing loss. Accordingly, we only included participants who reported before the screening that they had never or only occasionally had hearing complaints. Exploring how adults experience hearing screening will add valuable information to the discussion about whether or not adult hearing screening programmes should be implemented.

\section{Methods}

This study was conducted in the Netherlands between July 2011 and April 2013 and received ethical clearance from the Medical Research Ethics Committee of the Maastricht University Medical Centre. A qualitative approach was chosen because of the exploratory character of this study.

\section{Screening device}

We used the commercially available screening device Klangtester V3 Terminal, developed by Klangspektrum $\mathrm{GmbH}$. This device produces five tones $(1,2,4,6$, and $8 \mathrm{kHz})$ at an intensity level that slowly increases from $-5 \mathrm{~dB} \mathrm{HL}$ to $+75 \mathrm{~dB} \mathrm{HL}$. The person being tested wears circumaural headphones and is instructed to press a button as soon as he or she hears a tone. The next tone is played after the button is pressed or when a maximum intensity level has been reached. The five tones are first presented to the right ear and then to the left ear. The outcome is printed immediately after the last tone. The print-out shows a coloured graph with the rating of the hearing status (good/green, sufficient/yellow, decreased/orange, and insufficient/red) on the y-axis and the pitch on the $x$-axis (lowest tone on the left side; highest tone on the right side). Five points are shown on the graph for each ear (one point for each of the five tones) and these points are connected with a line.

In a study on cardiovascular prevention, Soureti and colleagues (2010) found that people were more likely to make lifestyle changes when told what their heart age was than when given a risk percentage. Based on Soureti's results, we expected that informing people with a hearing loss about their ear age would be more effective in stimulating them to seek help and start using hearing aids than showing them an audiogram. Researchers at the Dutch National Hearing Foundation (Nationale Hoorstichting) and the Audiological Centre at the Leiden University Medical Centre have developed a formula to calculate a person's ear age based on his or her hearing 
thresholds and the age-specific population norms (Spoor et al., 1969). They have adapted the Klangtester so that the ear age and its interpretation are printed as well.

Possible ear age outcomes are: $<30,30-45,53,58,62,65,68,71,74,77,80$, and $80+$. People with an ear age of 53 or lower are informed that their hearing is good; people with an ear age between 58 and 65 that their hearing is sufficient, people with an ear age between 68 and 74 that they probably have a hearing loss, and people with an ear age of 77 or higher that they have a hearing loss. People with an ear age of 68 or higher are advised to consider having extensive diagnostic testing.

\section{Sampling}

For recruitment, we cooperated with a Dutch company with a number of hearing aid shops in the south of the Netherlands where people can go for free hearing screening. The company regularly organises screenings at community locations such as supermarkets and weekly markets. The company also has access to the municipal personal records database and randomly calls older adults to offer their services and inform them of the possibility of making an appointment for a hearing test.

Employees at this company were instructed to ask customers prior to the screening whether they had ever had hearing complaints and, if so, how often. People who reported to never or only occasionally have hearing complaints but who screened positive for a hearing impairment (ear age $\geq 68$ ) were eligible for participation in the study. Eligibility was furthermore restricted to adults aged 40 years and above who had decided to do the hearing screening test for reasons other than doubts about their hearing and who had never before received a positive outcome on a hearing test.

The employees informed the eligible people about the study. If they were interested in participating, their contact details were given to the researcher $(A L)$ who then called them to make an appointment for a face-to-face interview within two weeks. Participant recruitment continued until data saturation was reached, that is, until new data no longer influenced the study results (Knudsen et al., 2012).

\section{Participants}

The details of the seven participants are presented in Table 1. Two of them lived alone (P6, P7); the others were married and lived with their husband or wife. Only the two youngest participants held a paid job (P4, P6). One participant was not screened with the Klangtester (P3), but had his hearing directly tested with pure-tone audiometry instead. We included him in the study because he tested positive for a hearing impairment and met all other inclusion criteria. 
Table 1. Participant details

\begin{tabular}{|c|c|c|c|c|c|}
\hline ID & Gender & Age & $\begin{array}{l}\text { Ear } \\
\text { age }\end{array}$ & $\begin{array}{l}\text { Hearing } \\
\text { complaints }\end{array}$ & Screening situation \\
\hline P1 & Female & 79 & $80+$ & Never & $\begin{array}{l}\text { P1 was approached by the hearing aid dispenser at the weekly } \\
\text { market. Because she failed the hearing screening test, she was invited } \\
\text { for a full audiometric assessment two weeks later in the hearing aid } \\
\text { dispenser's shop. There, her hearing loss was measured to be }>35 \mathrm{~dB} \\
\text { (best-ear pure-tone average at } 1,2 \text {, and } 4 \mathrm{kHz} \text { ). }\end{array}$ \\
\hline P2 & Male & 72 & 68 & Never & $\begin{array}{l}\text { P2 entered the hearing aid dispenser's shop to borrow a pencil, saw } \\
\text { the Klangtester, and asked if he could do the test. When he failed the } \\
\text { hearing screening test, he immediately requested rescreening (same } \\
\text { test), which he again failed. }\end{array}$ \\
\hline P3 & Male & 73 & & Occasionally & $\begin{array}{l}\text { P3 was persuaded over the telephone to make an appointment for } \\
\text { pure-tone audiometry at a local hearing aid dispenser shop. }\end{array}$ \\
\hline P4 & Female & 57 & 68 & Occasionally & $\begin{array}{l}\text { P4 participated in a hearing screening that was part of a driving } \\
\text { proficiency refresher course for seniors. }\end{array}$ \\
\hline P5 & Male & 73 & $80+$ & Occasionally & $\begin{array}{l}\text { P5 visited the open day of the hospital, saw the Klangtester, and } \\
\text { decided to do the test. }\end{array}$ \\
\hline P6 & Male & 52 & 68 & Never & $\begin{array}{l}\text { P6 had to undergo a hearing screening as part of an intake test for } \\
\text { participation in a drug trial. When he failed the hearing screening, he } \\
\text { immediately requested rescreening (same test), which he again failed. } \\
\text { Two weeks later, he went to the hearing aid dispenser's shop for a } \\
\text { different hearing screening test (Klangtester). }\end{array}$ \\
\hline P7 & Female & 64 & 68 & Never & P7 participated in a hearing screening organised at the supermarket. \\
\hline
\end{tabular}

\section{Data collection}

A researcher $(A L)$, who had been trained in interviewing, visited the participants at their homes for a semi-structured interview within two weeks after the screening. Written informed consent for participation in the study was obtained before the start of the interview. The interviews were audio-recorded and took place in the absence of a third party (partner or other housemate). The interviews had an open character, like a normal conversation, and took approximately one hour. A topic list was used to make sure all of the following topics were covered during the conversation: daily activities, subjective judgment of the participant's hearing status, reasons for participation in the screening, opinion about the screening test, opinion about the staff member (hearing aid dispenser), emotional impact of the screen outcome, associations with hearing impairment, actions following the screening, and views on hearing aids.

\section{Data analysis}

The audio-recorded interviews were transcribed verbatim and analysed using an inductive thematic analysis conducted from a realist perspective (Braun \& Clarke, 2006). An inductive thematic analysis involves a process that starts by assigning codes to sentences and fragments of the transcripts without trying to fit them into a preexisting code frame. Three researchers ( $A L, M J, L A)$ independently coded the interviews. After each transcript was coded, the researchers discussed the coding and reached consensus. They also discussed how different codes could be combined to form an overarching theme. Furthermore, themes identified earlier were reviewed to check whether the codes within a theme still cohered together meaningfully. If they did not, new themes were created or current themes were merged or split. The main 
themes, sub-themes, and sub-sub-themes were organised into a 'thematic map'. Each interview was transcribed ( $A L)$, coded (AL, MJ, LA), and discussed ( $A L, M J, L A)$ before a next interview was conducted (AL). A professional translation agency translated interview excerpts from Dutch into English for this report.

\section{Results}

We grouped the participants' reactions to the positive hearing screen outcome into three themes: (1) reflecting on the plausibility, (2) considering help-seeking and hearing aid uptake, and (3) emotional reaction. The themes are described below and are illustrated with interview excerpts. Table 2 shows the thematic map.

\section{Theme 1: Reflecting on the plausibility}

\section{Evaluation of the test validity}

Evaluating the test validity seemed to be a natural first reaction to a positive screen outcome. Participants judged the test validity positively if they had experienced the headphones to be soundproof (P3, P7), had not felt distracted during the screening (P3, P7), and had an asymmetric hearing loss (P6, P7). Participants who judged the screening test to be valid acknowledged and passively accepted the positive screen outcome (P3, P7), while the others seemed reluctant to do so.

"If I can hear the tones in my right ear, I should be able to hear them in my left as well. It's that simple." (P6)

Participants judged the test validity negatively if they had felt distracted during the screening by environmental noise (P1, P5, P6), environmental visual cues (P5), uncomfortable pressure of the headphones (P2), or the sound of their heartbeat, which they heard as a result of the occlusion effect of the headphones (P2). Other factors that contributed to participants judging the test validity negatively were a belief that the screening device was pre-programmed to make everybody fail the screening and purchase hearing aids (P1), the fact that only a few frequencies were tested (P4), the fact that it was a once-only assessment (P4, P5), and the fact that they did not know what to expect or what to focus on (P2, P4, P5).

\footnotetext{
"I don't really think that the test is reliable. It was like that once... I was told beforehand what type of sounds to expect. But still... I worried about how it would go, especially with the first ear." (P4)

"You can only know if it's correct if you've had the test done a few times. Once doesn't really tell you much." (P5)
}

Participants who did the same screening test a second time received the exact same outcomes as the initial screening (P2, P5). This changed their judgment of the test validity but did not necessarily help them acknowledge the outcome. 
she asked the others for complete silence and started the test again ... and I couldn't hear the high tones. She said to me: 'You only hear $40 \%$ in your left ear'. Oh well, all right, I'll live with that." (P6)

"It was only after the first test that I was told that, hey, listen, it's a kind of static sound that you will hear. I'd heard that the first time around, but didn't realise it was part of the test. During the second test, yeah, you pay more attention. But subconsciously I must have been paying attention during that first test, because the results were exactly the same... But l'm very happy with my hearing." (P2)

\section{Reflection on own hearing ability}

The positive screen outcome made the participants reflect on their hearing ability. They all tried to recall earlier signs of hearing loss, like sounds they no longer hear (e.g. doorbell, crickets), difficulty following television programmes without subtitles, examples of miscommunication, or remarks made by their significant others about their hearing. The outcome also motivated participants to test their hearing abilities in everyday listening situations (P1, P7). However, the participants did not always link the signs of hearing loss to their own hearing ability. Miscommunication, for example, was often ascribed to an unfavourable listening environment (e.g. loud background noise or distance) or to the communication partner mumbling. Generally, the more signs of hearing loss the participants recalled or observed and attributed to their own hearing ability, the more plausible they believe the positive test outcome to be.

"When I watch television, for example, and I'm focussed on that and my wife says something, I don't hear it. That's because my attention isn't focussed on her." (P5)

"My husband regularly warns me that I hear poorly. But well, he mumbles quite badly." (P4)

Question: "Have you thought about the screen outcome since then?" Answer: "I've thought about it. Because I think, well, I should try it and find out whether the right ear really is any worse than the left. I usually pick the phone up and hold it to my left ear, but after the hearing test, I tried picking up with my right ear. I deliberately thought: 'I should pick up with right'..." (P7)

For two participants without hearing complaints (P1, P6), the positive outcome raised doubts about their hearing ability and created a need for them to discuss it with significant others. Another participant without hearing complaints remained convinced that his hearing was functioning perfectly (P2).

"I spoke to my daughter about the test result. 'But mum', she said, 'I've never noticed you have bad hearing. Not at all!' And that one pays close attention to everything, you know. And she would tell me, hahaha." (P1)

Question: "Have you paid more attention to your hearing since the hearing test?" Answer: "No, I went home and moved on to other things. I haven't really thought about it since." (P2)

\section{Reflection on risk for hearing loss}

The outcome prompted the participants to reflect upon their risk for hearing loss. Some participants felt at risk for hearing loss because of old age (P3, P5, P7), noise exposure (P6), family history (P4), or a common cold (P6). These participants viewed the positive outcome as more plausible than those who did not feel at risk (P1, P2). 


\section{Theme 2: Considering seeking help and hearing aid uptake}

\section{Intention to seek help}

Two participants intended to consult a professional as a result of the screening: one because his wife wanted him to try hearing aids (P3) and the other because he did not believe aging was the cause of his hearing loss and thought perhaps his hearing could be fully restored by earwax removal or a different medical intervention (P6).

"I just think it's best to get it checked out, because maybe it can be fixed. If I don't go, I won't know." (P6)

\section{Willingness to use hearing aids}

All participants stated they would not use hearing aids until they developed hearing complaints that bothered them to such an extent that they perceived a need for hearing rehabilitation. At the time of the interview, the participants still felt their hearing was 'just fine' or 'not that bad'. However, there were signs that they trivialised their hearing difficulties, normalised their hearing loss, and lowered their hearing expectations.

\footnotetext{
Question: "How would you define 'hearing impairment'?" Answer: "Well, that you can't actually understand someone, right? That means nearby as well, right. This distance between you and me [distance of one meter in a quiet room]. I can understand you, but someone with hearing impairment can't." (P5)

"The difference between my ear age and my own age, well, in three months' time that'll only be three years. So... there's not much of a difference." (P7; chronological age $=64$ years; ear age $=68$ )

"If the doctor recommends a hearing aid, I'll try it. And if it makes things better, I'll have no problem with that. I don't really think it will make much difference though. And if that's the case, I won't wear it." (P3)
}

\section{Theme 3: Emotional reaction}

Surprise, disbelief, and incomprehension

Participants who felt that the positive outcome was not at all plausible reacted with surprise, disbelief, and incomprehension (P1, P2, P6).

"I was really surprised at how bad my test result was. How is it even possible? I can hear everything. I really can't imagine there's anything wrong with my hearing." (P1)

\section{Indignation}

Participants who judged the test validity negatively and were convinced that their hearing was perfect felt indignant about the outcome because they felt they were being unfairly labelled as hearing-impaired (P1, P2, P6). They insisted on rescreening under better conditions (i.e. silent environment, comprehensive instructions, or another measurement instrument) to prove that they were right. 
"The test was outside, and that doesn't work. I kept hearing all the noise from the market, so of course I couldn't hear the beeps." (P1)

"Well, my first reaction to the test result was actually: 'You can tell me whatever you like, but you're wrong. I can hear everything.' I told the doctor who performed the hearing test: 'Look, it's all well and good, but I can hear what people are saying three doors down. I can't hear that beep if those people over there are talking.' Then she asked the others for complete silence and started the test again." (P6)

\section{Disappointment}

Participants who suspected they had a hearing loss had hoped the test would prove otherwise. The positive screen outcome disappointed them (P4, P5). Disappointment was also reported by a participant who had no hearing complaints but tested positive for hearing loss (best-ear pure-tone average at 1, 2 and $4 \mathrm{kHz}>35 \mathrm{~dB}$ ) by a testing method she believed to be valid (P1).

\footnotetext{
"But well, it was silent at the hearing aid shop. The door was shut and it was sound proof. And even then the outcome was negative." (P1; audiometry in a soundproof room)
}

"I had hoped it wouldn't be so bad. I had hoped for some reassurance. But no such luck..." (P4)

\section{Concern about consequences of the outcome}

All participants reported that society nowadays expects people to take responsibility for their health and seek treatment or rehabilitation as soon as a health problem is diagnosed. They felt it was no longer socially acceptable not to seek rehabilitation (hearing aids) when diagnosed with a hearing loss. Consequently, two participants who suspected they had a hearing loss were reluctant to acknowledge the test outcome because they were concerned about the consequences (P4, P5): acknowledging the hearing loss would mean others would expect them to use hearing aids. They partly attributed the poor test outcome to a poor test validity but were not willing to have another hearing test because that might prove their hearing loss and mean having to deal with the consequences.

\footnotetext{
"But you can do something about it these days, with those hearing aids. So if it helps, you use one. For other people too, for their benefit that you... well... so you understand them." (P1)

Question: "If the hearing test had not been part of the driving proficiency test, would you have visited a hearing care professional for a free hearing test at some stage?" Answer: "I have thought about it before, but then I think 'oh, I can still manage' and 'it's not so bad'. But at the same time, I think 'I should get it done one day'. The consequence would be that I need a hearing aid and I'm not sure yet whether I want one." (P4)
}

\section{Concern about progression}

The two youngest participants were shocked by the positive screen outcome because their ear age was much higher than their chronological age (P4, P6). They were concerned about the progression rate of their hearing loss and about becoming completely deaf. 
"I wasn't happy to hear them say I had the hearing of a 68 year old. I thought 'blimey!'. What will it be like when I'm 68?!" (P6; chronological age $=52$ years)

\section{Concern about possible consequences of hearing loss}

The participants were concerned about what impact a (hypothetical) hearing loss might have on their future lives (P3, P4, P6, P7). They were aware that hearing loss could lead to them being excluded from participating in society and becoming socially isolated or lonely. The level of their concern depended on their beliefs about how severe the negative consequences would be on their social or professional functioning and on their confidence in the hearing aids being able to reduce those negative consequences.

"If the communication is no good, that affects your whole life, doesn't it?" (P3)

"A person with a hearing impairment has big problems if you ask me. It's annoying for other people too. They call twice and you don't respond. It would annoy me too if I had to call someone twice or three times and they didn't respond. I'd think that person was angry at me or something." (P6)

"My sister felt so isolated, but that disappeared thanks to her new hearing aids. You see her brighten right up again now she can hear more. Then I think 'maybe it would be a good idea to have one of those things'... so you can hear more easily when you're in company. If it really works, that is, because I'm not completely convinced yet." (P4)

\section{Emotional pain and sadness}

The participants associated hearing loss and hearing aids with being old, disabled, pitiful, and written off by society. The realisation that they were growing older and the belief that others would think less of them if they heard about their hearing loss caused emotional pain and sadness (P3, P4, P6). They believed that hearing loss was something to be ashamed of.

\footnotetext{
Question: "If you now noticed that someone has trouble hearing, would you recommend a hearing test?" Answer: "What would you say if we went out together and I was wearing a jacket or trousers that didn't suit me at all. What would you say?? Would you tell me? It's sensitive." (P3)

"I had a hard time accepting the test result." (P4)

"When I walk through town, I sometimes see elderly people with a hearing aid. But I'm talking about people who are well over 70. I'm 52. I don't think a 52 year old, who is really still so young, should be walking around with a hearing aid. I'd feel handicapped. Society writes you off. No, I won't do it. No way!" (P6)
}

"A hearing aid really does make you a senior citizen." (P4) 
Table 2. Thematic map

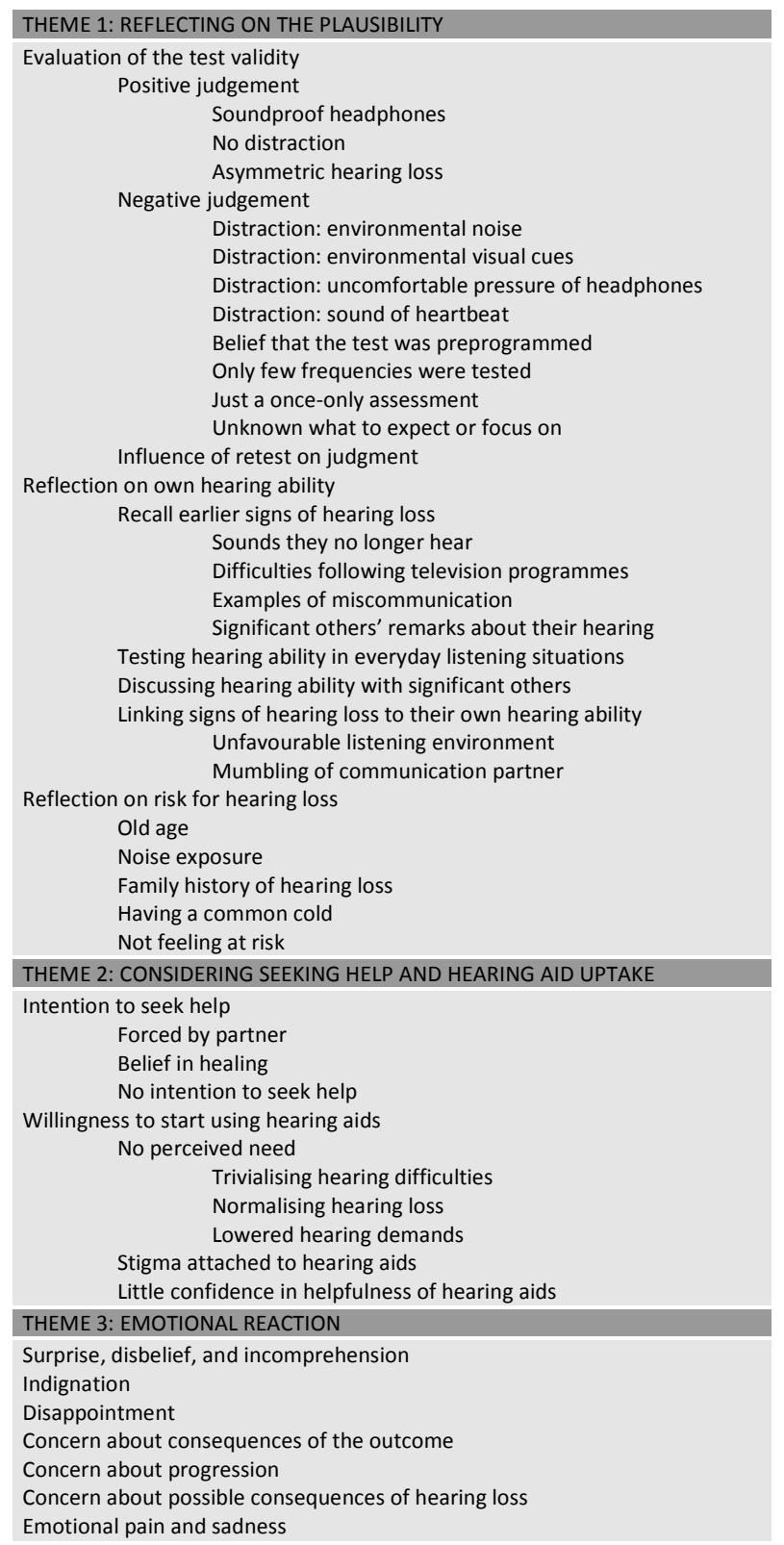




\section{Discussion}

This is the first study to explore adults' reactions to being labelled as hearing-impaired based on an objective hearing screening test. A positive screen outcome made people reflect on the test validity, their perceived hearing ability, their risk for hearing loss, their perceived need for professional help, and their willingness to start using hearing aids. Furthermore, the outcome evoked emotional reactions such as surprise, disbelief, incomprehension, indignation, disappointment, concern, emotional pain, and sadness. With the exception of indignation, these emotions were similar to the emotions reported by people diagnosed with a hearing impairment based on standard clinical diagnostic tests (Martin et al., 1989). Studies have shown that these emotions are part of the natural response to an increasing awareness of hearing loss (Engelund, 2006; Hindhede, 2012; Wallhagen, 2010; Weinberger, 1980). They should therefore be viewed as triggered but not caused by the screening. The fact that screening evoked such emotions supports the notion that screening can incite or accelerate the hearing loss recognition process.

The screening did not immediately prompt the participants to start using hearing aids. They all stated that they would not start using hearing aids until their hearing complaints bothered them to such an extent that they perceived a need for rehabilitation. A recent review of factors that influence help-seeking and hearing aid uptake also concluded that both are more strongly related to perceived complaints than to audiometric hearing thresholds (Meyer \& Hickson, 2012). This suggests that a subjective screening test might be better suited for testing people who could benefit from hearing aids than an objective screening test. However, one randomised controlled trial found that objective screening led to a larger increase in the 1-year incidence of hearing aid use than subjective screening (Yueh et al., 2010).

Some participants expressed indignation, an emotion that was particularly related to their beliefs about the test validity. This could probably have been prevented if the screening had taken place under better testing conditions. P1, for example, reported feeling indignant after the screening because the test had been carried out at a noisy market. However, her feelings of indignation were replaced by disappointment when her hearing was tested again two weeks later using standard clinical diagnostic tests in a soundproof room and again showed poor hearing (pure-tone average at 1, 2, and 4 $\mathrm{kHz}$ in the best ear $>35 \mathrm{~dB}$ ). The stories of $\mathrm{P} 2$ and $\mathrm{P} 6$ suggest that immediate retesting (with the same device) could reduce the level of indignation. In addition, indignation might have been prevented if a questionnaire had been used for screening instead of an objective hearing screening test.

\section{Methodological issues}

Although the seventh (last) interview did not generate any new codes or force us to adapt the thematic map, interviews with more people were needed to guarantee that we had identified all possible reactions. We did not interview any more people because it had proven difficult to recruit participants. 
Despite the recruiters' efforts, it took them 20 months to recruit the seven participants. There are several possible explanations for this slow recruitment. First, the number of eligible people might have been low because very few people without hearing complaints have their hearing tested in the absence of a systematic screening programme. Secondly, eligible people might not have been willing to participate because the test outcome may have been painful for them. Thirdly, eligible people might have refused to participate because they felt indignant about being unfairly labelled as hearing-impaired. Finally, eligible people might have been reluctant to participate because they feared the researcher would try to persuade them to purchase hearing aids.

We initially only aimed to include people without hearing complaints, but because the recruiters had difficulty finding people who met this criterion, we later decided to expand the inclusion criteria and include people who reported having hearing complaints occasionally. Although the study population was heterogeneous with regard to the presence of hearing complaints, the reactions of the two subgroups were largely similar. A minor difference between the subgroups was seen in their emotional reaction. Surprise, disbelief, incomprehension, and indignation were expressed solely by the participants without hearing complaints, while only those with complaints expressed concern about the consequences of the outcome.

This study was conducted from a realist perspective, which means that the interviewer tried to be as objective as possible and aimed to overcome socially desirable answers by concealing her value judgments about the interview topics. Furthermore, the fact that the interviewer was not a hearing care provider also reduced the chance of socially desirable answers.

\section{Ear age}

The participants regarded the discrepancy between their ear age and chronological age as a more valuable indicator of the severity of their hearing loss than the printed advice and graphical representation of the screen outcome. The ear age had a strong emotional impact on people whose chronological age was much lower than their ear age. Although the results did not prompt them to start using hearing aids immediately after the screening, because they felt they were too young, being informed about their ear age might help the hearing loss recognition and acknowledgement process. However, the ear age outcome had little impact on people whose chronological age was similar to their ear age. It reassured them that their hearing was normal for their age and did not prompt them to seek help even though they had been explicitly informed that they had a hearing loss and were advised to seek help. Older people with hearing complaints might decide not to seek help when their hearing turns out to be normal for their age, resulting in an even longer delay in help-seeking than in a non-screening setting. 


\section{Conclusion}

A positive outcome on an objective hearing screening test does not immediately prompt adults without hearing complaints to start using hearing aids. However, the results of this study suggest that objective hearing screening can incite or accelerate the hearing loss recognition process and might indirectly decrease the delay in helpseeking. One disadvantage of objective screening is that it can cause feelings of indignation. This might be overcome by using a highly accurate objective test under optimal testing conditions. 



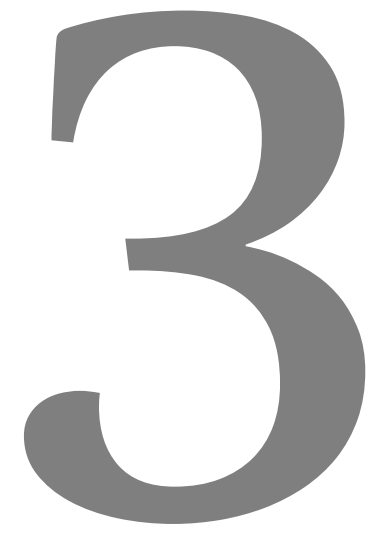

Qualitative interviews on the beliefs and feelings of adults towards their ownership, but non-use of hearing aids

Anouk M Linssen Manuela A Joore Rianne KH Minten Yvonne D van Leeuwen Lucien JC Anteunis

International Journal of Audiology 2013; 52(10): 670-677 


\section{Abstract}

This qualitative study aimed to increase our understanding of adults who own but not use hearing aids in order to get clues for non-use prevention and treatment. Individual face-to-face, semi-structured interviews were completed with 11 hearing aid owners (aged 54-80 years) who reported that they never or hardly ever used their hearing aids. Thematic analysis of the interviews showed that three types of non-users can be distinguished: non-users with no or mild hearing handicap ('Type A non-users'), nonusers with moderate to severe hearing handicap who attributed the non-use to internal factors ('Type B non-users'), and non-users with moderate to severe hearing handicap who attributed the non-use to external factors ('Type $C$ non-users'). Type $A$ non-users were indifferent about the non-use; Type $B$ non-users expressed feelings of self-annoyance, self-disappointment, and self-blame; and Type $C$ non-users expressed feelings of frustration, indignation, sadness, anger, powerlessness, and hopelessness. Disapproval of the non-use by significant others led to feelings of shame and guilt, but only for Type B non-users. The findings suggest a need for a patient-centred approach in hearing care to prevent and treat non-use of dispensed hearing aids. Non-use may partly be prevented by more careful consideration whether hearing aid fitting will be effective and by offering comprehensive counselling and hearing aid self-efficacy training during the hearing aid trial. 


\section{Introduction}

Untreated hearing loss has a variety of negative consequences, including social isolation, loneliness, and depression (Arlinger, 2003). Hearing aids can reduce these negative consequences and increase the quality of life (Chia et al., 2007; Chisolm et al., 2007; Joore et al., 2003b; Vuorialho et al., 2006). However, many adults with a hearing loss do not seek help, and even fewer take up hearing aids. In the Netherlands, for example, it has been reported that $56 \%$ of the adults (aged $\geq 55$ years) with a hearing loss of $30 \mathrm{~dB}$ or more (pure-tone average at $0.5,1,2$ and $4 \mathrm{kHz}$ in the better ear) seek professional help for their hearing loss, and that only $45 \%$ of these help-seekers take up hearing aids (Duijvestijn et al., 2003). Similarly, the Australian Blue Mountains Hearing Study revealed that just $61 \%$ of the adults (aged $\geq 50$ years) with a hearing loss of more than $25 \mathrm{~dB}$ (pure-tone average at $0.5,1,2$ and $4 \mathrm{kHz}$ in the better ear) sought professional help for their hearing loss (Schneider et al., 2010), and that only $51 \%$ of these help-seekers took up hearing aids (Hartley et al., 2010). Moreover, up to a quarter of the people who take up hearing aids never use them (Bertoli et al., 2009; Hartley et al., 2010; Kochkin, 2010).

Hearing aids are a great financial burden, either to the funding body (e.g. government or insurance) or to the hearing aid owner (if he or she paid for the hearing aids). However, hearing aid fitting is a cost-effective rehabilitation option for hearing loss (Joore et al., 2003a). This means that the gain in quality of life resulting from hearing aid use justifies the costs of the hearing aids. Non-use of dispensed hearing aids is undesirable for the health benefits missed as well as for the inefficient use of financial resources. The high proportion of non-users among hearing aid owners shows that the efficiency of hearing care is low.

Preventing the non-use of hearing aids might improve the efficiency of hearing care. Two qualitative studies recently found that hearing aid owners use their hearing aids when they feel disabled by their hearing loss or when their hearing loss limits normal participation in society (Laplante-Lévesque et al., 2013; Lockey et al., 2010). Thus, the perceived need for improved hearing seems to be the key factor in hearing aid use. Fitting hearing aids exclusively to people who perceive a need for improved hearing may limit or prevent the non-use of dispensed hearing aids.

Another way to improve the efficiency of hearing care might be treatment of people who own but do not use hearing aids ('non-users'). In order to help these people effectively, hearing care professionals need to understand the non-use. Therefore, several studies have been performed to find reasons for and predictors of non-use. According to a Swiss survey by Bertoli and colleagues (2009), the most frequently reported reasons for hearing aid non-use are: noisy disturbing situations (52\%), no perceived need (24\%), no or poor perceived benefit (23\%), unpleasant side effects (e.g. rashes, itching, pain, earwax accumulation; 19\%), poor sound quality (13\%), difficulties with management (9\%), and poor fit and comfort (9\%). A recent review by Knudsen and colleagues (2010) discussed predictors of hearing aid non-use. They concluded that significant non-use predictors included few self-reported hearing problems, low 
acceptance of the hearing loss, a negative attitude towards hearing aids, and manual dexterity problems. Other potential predictors addressed in this review included age, gender, living arrangement, hearing sensitivity, source of motivation, the hearing aid professional, personality, and expectations. However, scientific evidence for a relationship between these factors and hearing aid non-use was found to be either absent or inconsistent (Knudsen et al., 2010).

Knowledge about reasons for and predictors of non-use alone is not enough to come to an in-depth understanding of hearing aid non-users. Their beliefs and feelings with regard to the non-use are important as well. To date, it is still largely unknown how non-users feel about their non-use. Laplante-Lévesque and colleagues (2012b) reported on the perspectives of hearing-impaired adults with regard to the help-seeking and rehabilitation process. They noted that adults who stopped using their hearing aids expressed disappointment, embarrassment, and guilt, but did not expand on this topic. To our knowledge, feelings related to hearing aid non-use have never been explored exhaustively.

The aim of this study was to increase our understanding of adults who own but never or hardly ever use hearing aids by conducting an elaborate exploration of their beliefs (convictions) and feelings (emotions) about their non-use. Furthermore, we investigated the relationship between these beliefs and feelings. An in-depth understanding of the non-users might give clues for intervention.

\section{Methods}

\section{Study design}

This qualitative study was conducted in 2012 in the Netherlands. Data was collected using face-to-face semi-structured interviews and was analysed using inductive thematic analysis. The study had an explorative character; therefore, a qualitative approach was preferred over a quantitative approach.

\section{Sampling}

People were eligible for participation if they were between 50 and 80 years old and if they recognized themselves in the following description: "hearing aid owner who never or hardly ever uses his/her hearing aid(s)" (purposive sampling). The meaning of "never or hardly ever" was not specified in terms of objectively measurable units (e.g. hours per day or days per week); instead, the interpretation was left to the hearing aid owners themselves.

We aimed for a sample that was heterogeneous with regard to gender, age, living situation, and work status. Knudsen and colleagues (2012) recommend that a variety of recruitment sources be used to reach a heterogeneous population (maximum variation sampling). We used the following three recruitment sources: (1) seventeen 
general practitioners were asked to recruit eligible study participants among their patients, (2) an advertisement was placed in the newsletter of the senior citizens' association, and (3) a call for study participants was sent out by email to members of the researchers' personal networks. The email contained information about the study and encouraged the addressees to contact the researchers if they wanted to participate and fulfilled the inclusion criteria. The email recipients were also asked to forward the email to people in their networks and to talk about the study (word-of-mouth advertising) with acquaintances who they thought might be eligible but who did not have an email account.

\section{Participants}

Eleven volunteers were included in the study. The general practitioners together recruited two participants; the other nine participants responded to the email. None of the participants were direct members of the researchers' personal networks (firstdegree contacts), although the interviewer had met four of them before (seconddegree contacts, occasionally spoken to at social gatherings). The participants formed a heterogeneous population with regard to their socio-demographic characteristics. Participant details are presented in Table 1. All participants were fitted bilaterally and owned two digital behind-the-ear hearing aids. In the Netherlands, the hearing aid trial usually takes $8-12$ weeks. The hearing aid fit is verified during the trial using soundfield speech audiometry and real-ear insertion gain measurements.

Six participants (P1, P2, P4, P5, P8 and P10) were interviewed in the presence of their partner or another close relative; the other participants were interviewed alone. The participants chose whether or not a significant other was present during the interview. The researchers did not restrict the presence of others because it was thought to be unethical to ask the significant others to leave their own homes.

\section{Data collection}

A final-year medical student (RM) conducted the interviews after six weeks of training in audiology and interview techniques. The interviewer visited all participants in their homes. She briefly introduced herself as a student who was doing a scientific internship as the final task to obtain her Master's degree in Medicine. Before the interview started, the participants gave their written informed consent for audio-recording of the interview and for asking their hearing aid dispenser for a copy of their audiograms. The interviews had an open character, like a normal conversation, and took about 4560 minutes. All interviews started with the open question: "Can you tell us about your hearing and hearing aids?" A topic list was used to make sure that the participants' beliefs and feelings in relation to all of the following topics were covered during the conversation: hearing problems without and with hearing aids, hearing help-seeking, hearing aids (including expectations, advantages, disadvantages, and handling), and the roles of significant others and hearing care professionals. 


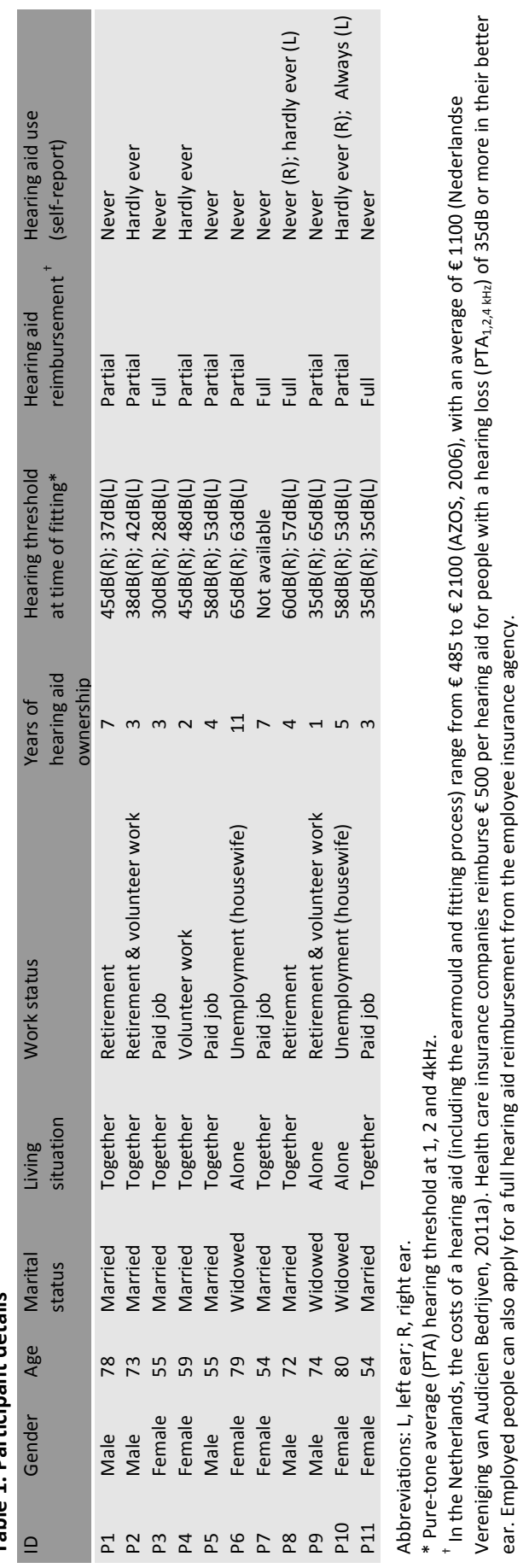




\section{Data analysis}

The audio-recorded interviews were transcribed verbatim. The transcripts were analysed using inductive thematic analysis, conducted from a realist perspective (Braun \& Clarke, 2006). Thematic analysis involves searching across the interview transcripts to find repeated patterns of meaning. It is a flexible research tool due to its independence from theory (Braun \& Clarke, 2006). Since the present study aimed to identify relationships between beliefs and feelings with regard to hearing aid non-use (the meaning of non-use), thematic analysis was judged to be a useful analysis method. An inductive (data-driven) approach was considered to be most appropriate, since little was known beforehand about the beliefs and feelings of hearing aid non-users (Knudsen et al., 2012).

Inductive thematic analysis starts by adhering codes to sentences and fragments of the transcripts, without trying to fit them into a pre-existing code frame. Two researchers (AL \& RM) independently coded the interviews. After each transcript was coded, the researchers came together to discuss the coding and come to consensus. During these meetings, they also discussed how different codes might combine to form an overarching theme. Furthermore, themes that were identified earlier were reviewed to check whether the codes within a theme still cohered together meaningfully. If not, new themes were created or previous themes were merged or split. Main themes, sub-themes, and sub-sub-themes were organized in a 'thematic map'.

Each interview was transcribed (RM), coded ( $A L$ \& RM), and discussed ( $A L$ \& $R M)$ before another interview was conducted (RM). To ensure trustworthiness, a third researcher $(\mathrm{YL})$ coded every third interview to test whether the resulting codes fitted in the created thematic map or whether it had to be adapted. The ninth transcript was the first that did not bring in new codes or force adaption of the thematic map, which implied data saturation (Knudsen et al., 2012). Two more participants were included to ensure data saturation. An audiologist (LA) who was not involved in the data analysis process performed an external audit to evaluate whether the findings, interpretations, and conclusions were supported by the data. The audit was based on reading all the interview transcripts and examining the thematic map; it did not result in changes to the themes, sub-themes, or sub-sub-themes.

This study was conducted in Dutch. For this report, a professional translation agency translated the illustrating interview excerpts into English.

\section{Results}

\section{Beliefs and feelings}

The participants expressed feelings of indifference, self-annoyance, self-blame, selfdisappointment, shame, guilt, frustration, indignation, anger, sadness, powerlessness, and hopelessness toward their hearing aid non-use. How they felt about their non-use was related to three main themes: (1) their beliefs about the severity of their hearing 
handicap with and without hearing aids, (2) their beliefs about whom or what was responsible for the non-use, and (3) their beliefs about their significant others' attitudes towards the non-use. Within these three main themes, 10 sub-themes and 40 sub-sub-themes were identified (Table 2). Figure 1 depicts a global overview of the relationships between the beliefs and feelings of hearing aid non-users. The three themes are described below and are illustrated with interview excerpts.

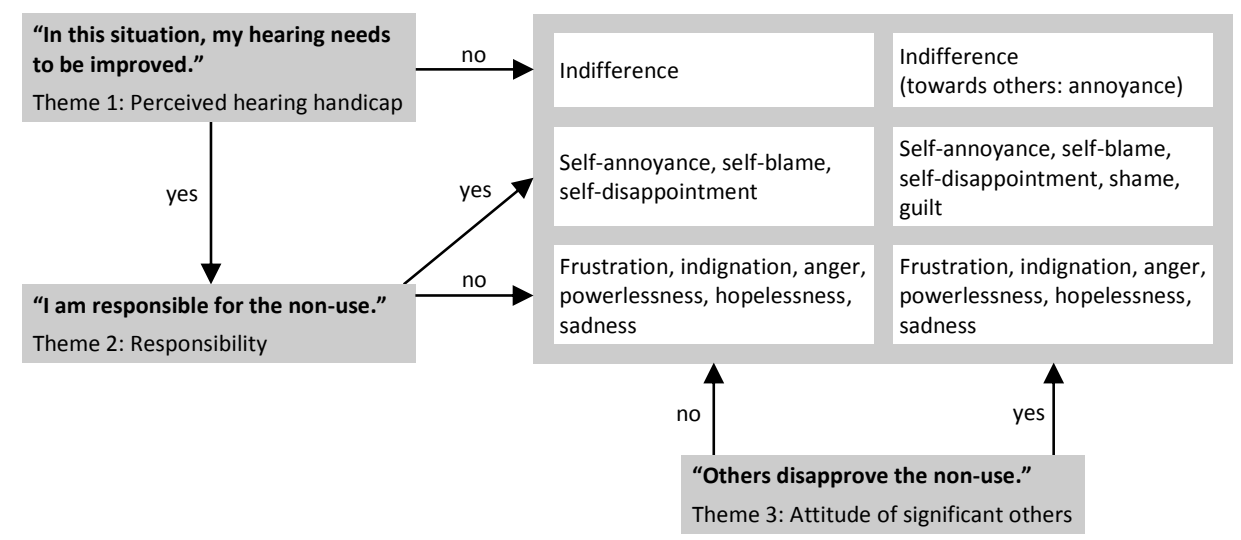

Figure 1. Relationships between hearing aid non-users' beliefs and feelings about their non-use 
Table 2. Thematic map

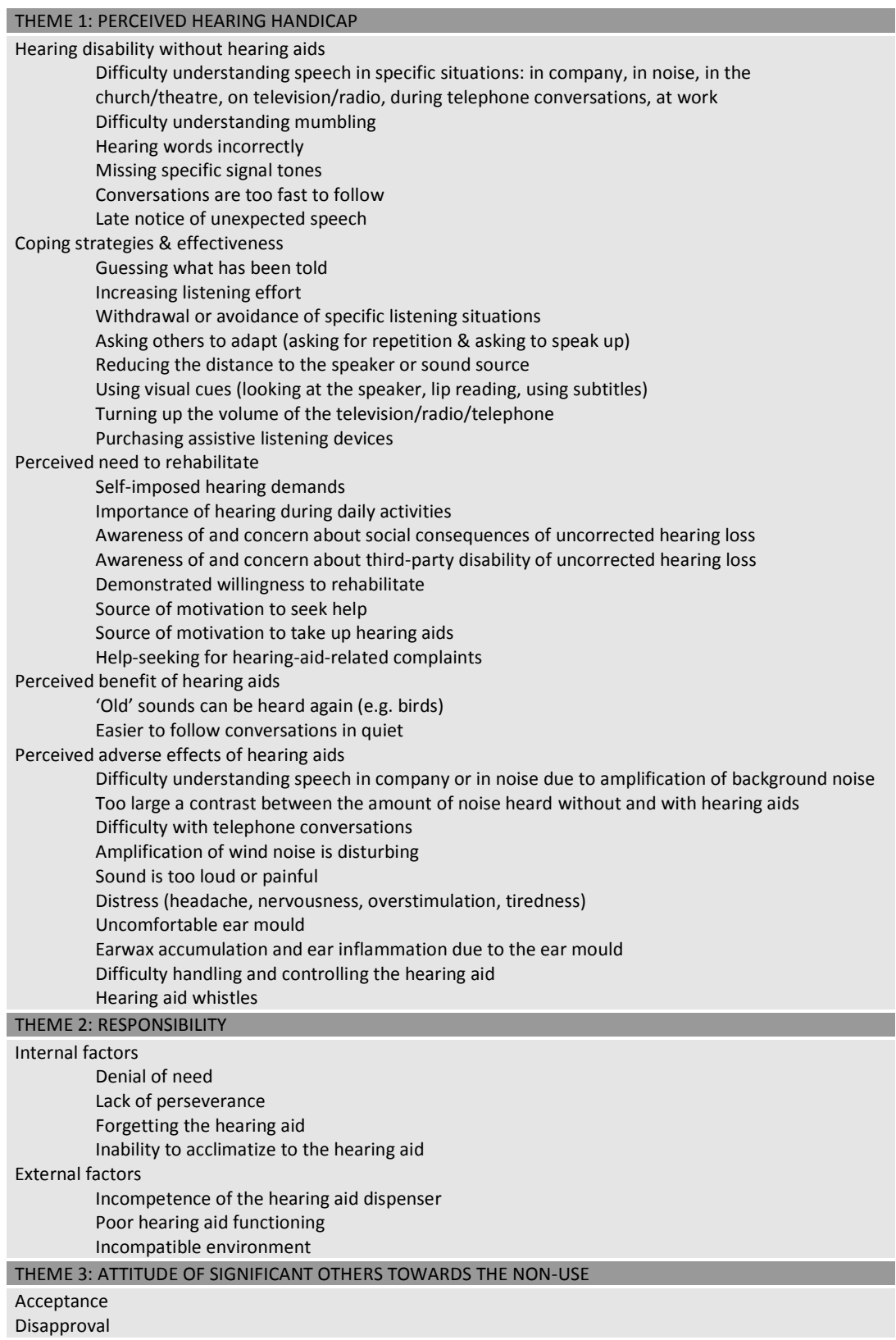




\section{Theme 1: Perceived hearing handicap (with and without hearing aids)}

Participants talked about their hearing disability without hearing aids, the effectiveness of coping strategies to reduce hearing handicap, the perceived need and demonstrated willingness to rehabilitate, and the perceived benefits and adverse effects of hearing aids on their hearing handicap (Table 2). People who perceived only a mild handicap from their hearing loss tended to feel rather indifferent about their non-use. They tried the hearing aids for a while, but perceived the disadvantages of the hearing aids to be greater than the disadvantages of their hearing loss. They decided for themselves that they could hear well enough by using communication strategies and that they still could manage without hearing aids. They probably would not even have started a hearing aid trial if their significant others had not pressured them to visit a hearing care professional.

Question: "What do you do when you can't hear someone?" Answer: "Nothing. Just let them go on. I don't need to hear everything. Hehehe." (P6)

"I mean, if I look at you I can understand you, can't I?! So, uh..." (P9)

People who perceived a more severe handicap from their hearing loss expressed feelings of frustration and sadness towards their non-use. They felt a strong need to improve their hearing and had consulted a hearing care professional on their own initiative, but the hearing aids did not help them as much as they had hoped. They stopped using the hearing aids because they did not reduce their hearing handicap.

"A conversation in a group is simply a disaster when I am wearing that thing. Often, I pull it off. And, what I find regrettable is that ... uh... I am letting myself become secluded." (P1)

"With or without a hearing aid, I am not able to communicate decently with anyone, which is quite awful." (P8)

\section{Theme 2: Responsibility}

\section{Internal factors}

The participants expected that the hearing aids would reduce their hearing disabilities. However, the hearing aids were generally perceived to be less beneficial than initially hoped for or to have disadvantages that were worse than expected. As a result, the participants felt disappointed. Some people said they had faith in the hearing aid dispenser's claim that it is a matter of acclimatization and they had accepted that hearing aids cannot fully restore their hearing. They tended to feel responsible for their non-use. P3, for example, blamed herself (self-blame) for her denial of her need for improved hearing.

"I think I will start wearing them again when it becomes really troublesome. At the same time, I think: I do not need them yet. But that is nonsense, because there are situations in which I really do need them. Stop." (P3) 
Many non-users blamed themselves for their lack of perseverance during the hearing aid trial and acclimatization period. They expressed irritation with their own behaviour (self-annoyance) or disappointment in themselves (self-disappointment).

\footnotetext{
"I am irritated with myself and I think: Yeah, shit. Now I didn't hear it again, now I have to ask someone to repeat it. You know. Hehehe. But still I don't wear these things ... sometimes I don't understand myself." (P3)

"Maybe, if I had persevered in the beginning, it might have worked. But if you are not $100 \%$ behind it, then it won't work of course. Hehehe. Maybe then, I was too easy." (P7)
}

Self-annoyance was also seen in people who blamed themselves for not having taken the hearing aids with them into a situation where they knew that the hearing aids would be helpful.

"Sometimes, there are situations that I think: Oh, stupid, stupid, stupid, that I didn't put them in. Yes, then you get fed up with yourself." (P4)

Some people blamed themselves for being incapable of acclimatizing to the hearing aids. P2, for example, believed he was responsible for the hearing aid use himself, but at the same time he felt that it was out of his control. He expressed mixed feelings towards the non-use.

"It should work effectively, and that is what they do. What else could I want? [...]. Everything is amplified, but you pick up so much more sound that it becomes a mixture of noises. And it's hard to filter out what is important. I tried it for a year, but was unsuccessful. On the one hand, I blame it on myself, while on the other hand, I think, well..." (P2)

\section{External factors}

Some people did not trust the hearing aid dispenser's claim that it is a matter of acclimatization. They blamed the hearing aid dispenser for being incapable of adjusting the hearing aids accurately to their hearing loss or for advising them to buy the wrong hearing aids. They believed that the hearing aid dispenser's lack of professionalism had kept them from benefitting from hearing aids and, therefore, he was responsible for the non-use. People who held the hearing aid dispenser responsible for their non-use expressed feelings of indignation, frustration, anger, powerlessness and hopelessness, especially if they perceived a severe hearing handicap. People who held the hearing aids responsible for the non-use expressed feelings of powerlessness and frustration as well. Again, particularly people who perceived severe hearing handicap expressed such negative feelings. People with milder hearing handicaps were still rather indifferent about their non-use, even when they blamed the hearing aid dispenser or the hearing aids for their non-use.

\footnotetext{
"People do not listen. They do not communicate. The lady [hearing aid dispenser] only wants to sell me new things. So she turns down the amplification of my hearing aids so that I have to come back to her. But I won't be going back!" (P5)

"That man is totally incompetent. He can't adjust the hearing aids in such a way that the rustle is gone." (P8)
} 
"For months I have had an inflammation in the ear. That is why I am not wearing it. [...]. The hearing aid is the true culprit. It causes me to have inflammations and have earwax get stuck." (P1)

\section{Theme 3: Significant others' attitudes towards the non-use}

Pressure from significant others to use the hearing aids was found to evoke various reactions and feelings in the non-users. $\mathrm{P} 4$, for example, used the hearing aids in most (but not all) situations in which she thinks it is important to hear well, which was on average two hours per day. She believed that she used them reasonably effectively. She also believed that her significant others expected her to use the hearing aids all day long. Not meeting their expectations made her feel as if she had failed them, which went hand in hand with feelings of shame and guilt.

"And it is wrong, I know. I should always wear them, but... [...]. And, maybe it is weak of me not to persevere, I don't know. Look, normally in the daytime I am home alone. Well, they are really not needed then." (P4)

P6 also felt pressured by others to use the hearing aid. She did not experience hearing aid use as something that could be beneficial for her, but as just another obligation that others had imposed on her. She did not feel like obeying anymore. Not using the hearing aids seemed to make her feel in control of her own life.

"I always say: they are useless bloody things. Why? Pff... I think that is because of the obligation that is imposed on you: you should wear them, you know." Question: "Because other people say so?" Answer: "I think so, I don't know. But actually, that is not my problem. I am me, right?! [...]. I already have enough obligations. Honestly! And then at my age. They won't grant you a moment's peace." (P6)

P10 used one hearing aid all day long. When she sat with visitors around a table, she sometimes put in the second hearing aid because significant others have told her it is better to do that and to make them stop badgering her about it. Despite the pressure from her significant others, she was rather indifferent about the non-use of the second hearing aid because she did not notice any benefit from it.

\section{Discussion}

This is the first study that elaborately reports on the beliefs (convictions), feelings (emotions), and the relationship between the beliefs and feelings of adults who own but do not use hearing aids with regard to their hearing aid non-use. A large variety of feelings were found; they were related to beliefs about perceived hearing handicap, responsibility, and the attitudes of significant others. Three types of non-users can be distinguished broadly: non-users with no or mild hearing handicap ('Type A nonusers'), non-users with moderate to severe hearing handicap who attributed the nonuse to internal factors ('Type B non-users'), and non-users with moderate to severe hearing handicap who attributed the non-use to external factors ('Type $C$ non-users'). Type A non-users were indifferent about the non-use; Type B non-users expressed feelings of self-annoyance, self-disappointment, and self-blame; and Type $C$ non-users expressed feelings of frustration, indignation, sadness, anger, powerlessness, and 
hopelessness. Disapproval of the non-use by significant others led to feelings of shame and guilt, but only for Type B non-users. The three types are not mutually exclusive, because the perceived handicap and need for improved hearing are context dependent. Namely, someone can be a Type A non-user in one situation (e.g. when home alone), but a Type B or C non-user in another situation.

\section{Methodological issues}

The presence of a significant other during the interview might have inhibited some of the participants from talking freely and honestly about their beliefs and feelings. The participants were free to decide whether or not they wanted a significant other to be present during the interview. Participants who have difficulty talking about their true beliefs and feelings in the presence of a significant other probably chose to be interviewed alone. Consequently, the influence of social desirability due to the presence of a significant other would be limited. This notion is supported by the fact that participants who were interviewed alone as well as participants who were interviewed in the presence of a significant other reported negative feelings as a result of the significant other's disapproval of the non-use.

In a qualitative interview study, the participants can also adapt their answers to what they think the interviewer wants to hear. The present study was done from a realist perspective, which implies that the researchers believed that what the participants said was the reality, and not some idea about reality constructed by interaction between the participants and the interviewer. In practice this means that the interviewer tried to be as objective as possible and aimed to overcome socially desirable answering by concealing her value judgments about the interview topics. Furthermore, the fact that the interviewer was not a hearing care provider also reduced the chance of socially desirable answering. However, the results may be biased slightly, in any direction, due to the fact that some of the participants were second-degree acquaintances of the interviewer.

The transferability of the results to non-users who did not participate in the study is warranted in two ways. First, maximum variation sampling was used to select a heterogeneous study population. Second, data collection did not stop before data saturation was reached. Consequently, the results are likely to be applicable to other hearing aid non-users in the Netherlands and abroad. The prevalence of the different types of nonusers will differ between countries, due to differences in the hearing aid provision and reimbursement systems. Type A non-users, for example, might not be found in countries where hearing aid provision or reimbursement is based on the assessment of hearing handicap.

\section{Comparison with literature}

So far, feelings about hearing aid non-use have only been addressed briefly by Laplante-Lévesque and colleagues (2012b), who found that non-users expressed feelings of disappointment, embarrassment, and guilt. The participants in this study 
expressed these feelings as well. Lockey and colleagues (2010) interviewed regular hearing aid users and found that their decisions on whether or not to use the hearing aids in a specific situation depended on consideration of meaningful participation with and without the hearing aids. In this study, beliefs about meaningful participation are captured within Theme 1 (perceived hearing handicap). Lockey and colleagues (2010) also found that hearing aid non-use was associated with purposeful engagement in the non-amplified world, the transfer of the burden of communication to others, and the use of other communication strategies. The latter two themes were recognized in the present study too, but the first theme (purposeful engagement in the non-amplified world) was not. Our participants did not report on advantages of having a hearing loss. The fact that Lockey and colleagues (2010) interviewed regular hearing aid users while the participants in our study were hearing aid non-users, might explain the difference.

Perceived hearing handicap is the only factor that is consistently found to be related to help-seeking, hearing aid uptake, hearing aid use, and hearing aid satisfaction (Knudsen et al., 2010). This is the first study to show that perceived hearing handicap is also related to how hearing aid owners feel about their non-use, with more severe hearing handicap being associated with more negative feelings. The emotional consequences of non-use, in cases of moderate to severe hearing handicap, depended on the non-user's sense of responsibility. Previously, it had been found that hearingimpaired adults who feel responsible for and in control of their health are more likely to seek help, to take up hearing aids, and to have more positive hearing aid outcomes than adults who do not feel responsible for and in control of their health (Cox et al., 2005; Garstecki \& Erler, 1998; Laplante-Lévesque et al., 2012a). Sense of responsibility has not been related to feelings about hearing aid non-use before. However, the feelings of Type B non-users are similar to the feelings of people who are aware of their hearing loss but have not sought help yet (Engelund, 2006).

\section{Empirical findings in relation to theory}

The present finding that one's sense of responsibility influences one's feelings about a behaviour is in accord with Weiner's attribution theory of motivation (Weiner, 2012). This theory assumes that people always try to attribute the cause of success or failure (here: non-use) to someone or something. Three causal dimensions are distinguished: locus, stability, and controllability. The locus dimension refers to whether the cause is perceived as internal or external to the individual. The stability dimension refers to whether the cause is stable or unstable across time and situations. The controllability dimension refers to whether the cause is perceived as being under the control of the individual or not. In general, people who believe that the cause of failure is internal, unstable, and controllable tend to blame themselves. On the other hand, people who believe that the cause of failure is external, stable, and uncontrollable tend to feel hopeless (Weiner, 2012).

Originally, this study only identified the locus dimension; the sub-theme 'Responsibility: internal factors' was comparable to Weiner's internal locus and the sub-theme 'Responsibility: external factors' was comparable to external locus. However, when the 
data was reviewed (post-hoc analysis) with the three causal dimensions in mind, the stability and controllability dimensions were found in the data as well (Table 3). The stability dimension was recognized in that participants attributed their non-use to their cognitive incapability to acclimatize to the hearing aids (stable) or to a temporary ear inflammation (unstable). The controllability dimension was recognized in that participants attributed their non-use to their lack of perseverance (controllable) or to the incompatible environment (uncontrollable). Participants' feelings were in line with what would be expected based on Weiner's attribution theory. Knowledge of the nonusers' beliefs about locus, stability, and controllability can assist in deciding how each of them can best be helped.

Table 3. Causal dimensions of Weiner's attribution theory with examples from the present study

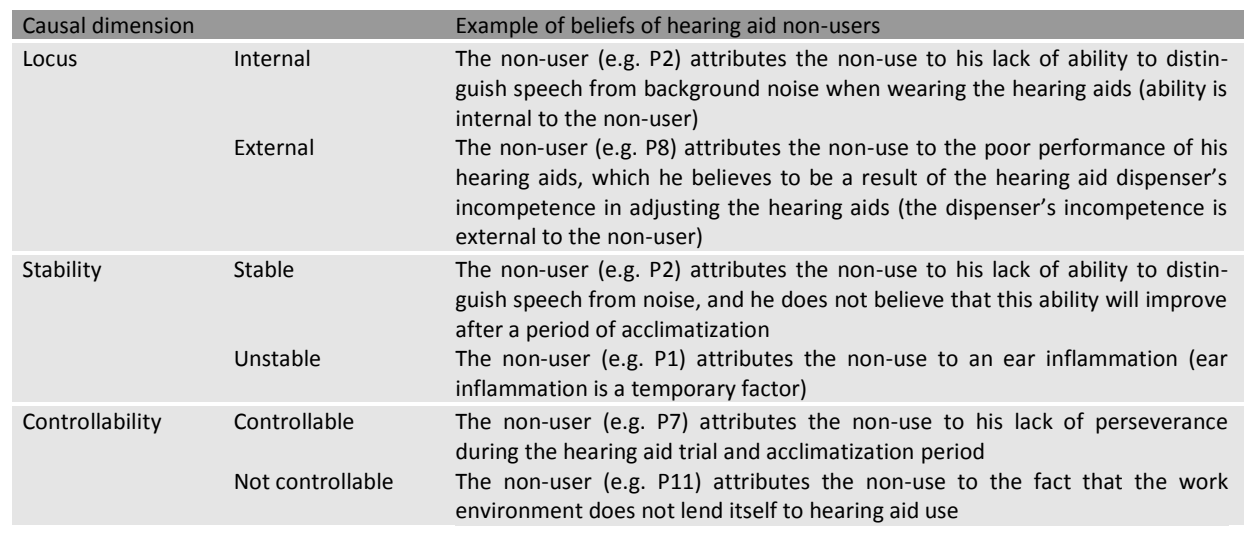

\section{Clinical implications}

It is difficult to help non-users because many of them do not present themselves to hearing care professionals after the hearing aid purchase. In the Netherlands, some hearing aid dispensers send hearing aid owners an annual invitation for a free control visit. However, this does not seem to be a successful way to identify non-users, as all our participants had received such an invitation but none of them had responded to it. Type A non-users did not see the point of a control visit because they did not intend to use their hearing aids anyway. Type B non-users found control visits unnecessary because they believed that their hearing aids were functioning properly. Type $C$ nonusers had returned to their hearing care dispensers many times but were dissatisfied about the provided help. They did not respond to the invitation because they believed that their hearing aid dispensers were unable to help them adequately. The sections below describe how non-users can be helped (if identified) and - maybe even more important - how future hearing aid seekers can be prevented from becoming nonusers. 


\section{Type A non-users}

For non-users who do not perceive a strong need for improved hearing, hearing aid non-use itself has no emotional consequences, although the pressure from others to use the hearing aids can be perceived as annoying. Hearing aid owners define optimal use as the use of the hearing aid in situations where they perceive a need for improved hearing (Laplante-Lévesque et al., 2013). Based on this definition, it is not surprising that people who perceive no or only a mild hearing handicap are indifferent about the non-use. Forcing them to use their hearing aids is unlikely to result in benefit, either personal or societal. Hearing care professionals should accept and respect that Type $A$ non-users prefer not to receive further professional attention. Communication training might be a more appropriate way of rehabilitation, but this option was not discussed with the participants. If communication training had been offered as well, some of the people who took up hearing aids - especially those who perceived only mild hearing handicap - might have chosen communication training instead of hearing aid fitting (Laplante-Lévesque et al., 2012a).

To prevent future hearing help-seekers from becoming Type A non-users, hearing care professionals should inform them about all available rehabilitation options (e.g. hearing aids, communication training programmes, assistive listening devices, doing nothing) and decide together about the best option. In particular, the perceived severity of the hearing handicap, not the audiograms, should guide this shared decision making process. For people who perceive no or only mild hearing handicap, doing nothing is probably the best option.

\section{Type B non-users}

For non-users who express feelings of self-annoyance, self-disappointment, or selfblame with regard to the non-use, internal factors that restrain hearing aid use require particular attention during the audiological consultation. Type B non-users seem to have a low hearing aid self-efficacy: they have little confidence in their abilities to be successful hearing aid users. Lack of perseverance is also an indication of low hearing aid self-efficacy (Smith \& West, 2006). Therefore, Type B non-users might benefit from hearing aid self-efficacy training to enhance their self-confidence with regard to hearing aid use. By building hearing aid self-efficacy, non-users will be more motivated to adjust to hearing aids, persevere when hearing aid difficulties arise, and apply more effort toward successful hearing aid use. Smith and West (2006) described a number of strategies for enhancing hearing aid self-efficacy, including role plays and motivational coaching by hearing care professionals or significant others.

To prevent future hearing aid seekers from becoming Type B non-users, their hearing aid self-efficacy can be measured at the time of hearing aid fitting, using the MARS-HA (Measure of Audiologic Rehabilitation Self-Efficacy for Hearing Aids) questionnaire developed by West and Smith (2007). Knowing a person's level of hearing aid selfefficacy can assist the hearing care professional in determining whether or not the person needs intensive counselling or self-efficacy training during the hearing aid trial in order to become a successful hearing aid user. 


\section{Type C non-users}

For non-users who express feelings of frustration, indignation, sadness, anger, powerlessness, or hopelessness with regard to their non-use, external factors that restrain hearing aid use require special attention during the audiological consultation. For example, someone who blames a hearing aid dispenser can best be advised to visit a hearing care dispenser in whom he or she has more faith. Furthermore, if the external factor that restrains hearing aid use is truly stable and uncontrollable (e.g. incompatible environment), hearing care professionals can best propose rehabilitation options other than hearing aids, such as communication training or assistive listening devices.

To prevent future hearing aid seekers from becoming Type $\mathrm{C}$ non-users, the hearing aid dispenser should put considerable effort into gaining the patient's trust. It is important that the hearing aid dispensers show a high level of professionalism, listen carefully to the hearing aid seeker, take his or her complaints seriously, provide accurate and comprehensible information, and involve the hearing aid seeker in the selection and fitting process.

Pressure from significant others to use the hearing aids can negatively influence the emotional well-being of some hearing aid non-users. However, whether or not hearing care professionals should discourage significant others from pressuring the non-users depends on the potentially positive effect on hearing aid use and benefit. To date, pressure from others has only been reported to increase hearing help-seeking and hearing aid uptake (Laplante-Lévesque et al., 2013). There is no evidence yet that pressure from others also increases hearing aid use with a resulting increase in hearing aid benefit. To examine the effects from pressure of significant others on hearing aid use and benefit, a quantitative study is needed that includes both hearing aid users and hearing aid owners who do not use their hearing aids.

\section{Conclusion}

Adults who own but do not use hearing aids have different beliefs and feelings about hearing aid non-use. It can have negative emotional consequences, especially for nonusers who believe that their hearing needs to be improved. Together with the missed health benefits and the inefficient use of financial resources, the negative emotional consequences of non-use underscore the importance of preventing and treating hearing aid non-use. A patient-centred approach is needed to provide each non-user with the best help. 



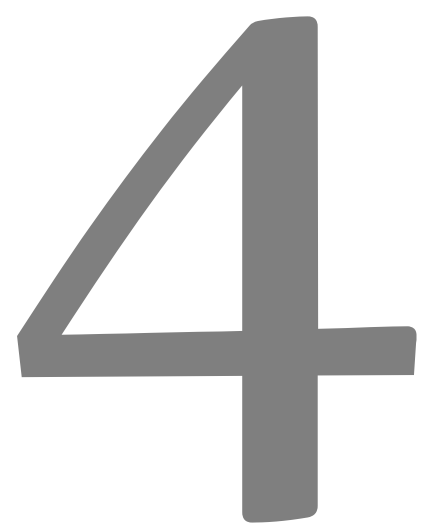

The effects and costs of a hearing screening and rehabilitation programme in residential care homes for the elderly in the Netherlands

Anouk M Linssen

Manuela A Joore

Eric JJM Theunissen

Lucien JC Anteunis

American Journal of Audiology 2013; 22(1): 186-189 


\section{Abstract}

This study describes the effects and costs of hearing screening and rehabilitation in residential care homes for the elderly. It was hypothesised that offering an in-house hearing screening and rehabilitation programme would be an effective strategy to increase hearing aid ownership among the residents. All 705 residents of 8 residential care homes in the Netherlands were invited to participate in a hearing screening (puretone audiometry) and rehabilitation (hearing aids) programme. A total of 243 residents (34\%) participated in the screening, 222 (91\%) of whom had hearing loss. Ninety-one $(41 \%)$ of the screen participants with hearing loss started rehabilitation, which was successful for 50 (55\%) of them. Hearing aid ownership among the residents with hearing loss increased from $28 \%$ at the start of the programme to $33 \%$ at the end. The costs were $€ 1896$ per successfully rehabilitated resident. Hearing aid trials and hearing aids together accounted for $83 \%$ of the total costs. The effectiveness of the programme was limited, as hearing aid ownership increased only slightly. Cost reduction measures should focus on decreasing the number of unsuccessful hearing aid trials. 


\section{Introduction}

Approximately $90-95 \%$ of elderly care home residents have a pure-tone average hearing loss (at 0.5, 1, 2, and $4 \mathrm{kHz}$ ) $>25 \mathrm{~dB} \mathrm{HL}$ in the better ear (Stumer et al., 1996; Tolson, 1997; Tsuruoka et al., 2001). Hearing loss negatively affects communication with nursing staff, visitors, and other residents, which can result in social isolation and depression (Brink \& Stones, 2007; Mahoney, 1992; Tsuruoka et al., 2001). Although hearing aids can partly address the communication problems of care home residents (Jupiter \& Spivey, 1997), only $12-17 \%$ of the residents own hearing aids (CohenMansfield \& Taylor, 2004; Tolson, 1997). It has been recommended that hearing screening be implemented to increase hearing aid ownership and use in residential care homes (Adams-Wendling et al., 2008; Cohen-Mansfield \& Taylor, 2004).

This study describes the effects and costs of a hearing screening and rehabilitation programme that was organised in eight residential care homes for the elderly in the Netherlands in 2010. We anticipated that easy accessibility would be a great facilitator for participation in the programme; therefore, the hearing care professionals visited the homes. The results provide valuable information for hearing care professionals and health care policy makers who strive for evidence-based practice and optimal allocation of resources.

\section{Methods}

\section{Study population}

We invited all of the residents $(\mathrm{N}=705)$ of eight residential care homes for the elderly to participate in the hearing screening and rehabilitation programme, independent of whether or not they already owned hearing aids. Dutch residential care homes typically house elderly people who are unable to live independently but who do not need intensive nursing care. Depending on the needs of the resident, the homes provide meals; housekeeping; and assistance with activities of daily living, such as eating, dressing, and personal hygiene.

\section{Programme design}

\section{Recruitment}

Residents were informed about the programme through information brochures, announcement posters, an advertisement in the care home paper, and personal communication (door-to-door visits by an audiology assistant). 


\section{Screening}

The screening consisted of air-conduction pure-tone audiometry that was performed by an audiology assistant. Residents with an average hearing loss (at 1, 2, and $4 \mathrm{kHz}$ ) of $>35 \mathrm{~dB} \mathrm{HL}$ in their better ear were told that they had hearing loss. This definition of hearing loss was chosen because, in the Netherlands, only persons who meet this criterion are entitled to partial hearing aid reimbursement. All screen participants were asked if they wanted an appointment with an ear, nose and throat (ENT) specialist. For those who wanted to consult the ENT specialist, speech audiometry was performed by the audiology assistant immediately after the screening.

\section{Rehabilitation}

The ENT specialist's consultations took place within two weeks after the screening. The ENT specialist was responsible for clinical examination of the ear, earwax removal, diagnostics of ear pathology, interpretation of hearing test results, communication with the resident regarding rehabilitation options (hearing aid fitting, adjustment of a previously fit hearing aid, or no rehabilitation), and prescription of hearing aids to the residents who were willing to try them. Furthermore, the ENT specialist referred residents to the audiologist when there was a need for additional counselling, a problem in accepting hearing loss, limited willingness to use hearing aids, severe hearing loss (best-ear pure-tone average at 1, 2, and $4 \mathrm{kHz} \geq 70 \mathrm{~dB} \mathrm{HL}$ ), poor speech discrimination ( $<70 \%$ for monosyllables), severe visual impairment, poor manual dexterity, or dementia. These referral criteria are set out in the Dutch guidelines for hearing care professionals (Nationaal Overleg Audiologische Hulpmiddelen, 2009). The audiologist is a clinical physicist who holds a post-academic degree in audiological and psychosocial rehabilitation. Whereas the ENT specialist discusses rehabilitation by hearing aid fitting only, the audiologist offers many more rehabilitation options, including communication training, psychosocial support, and assistive listening devices.

Residents who received a hearing aid prescription could consult the hearing aid dispenser, who was present in the home on the same day as the ENT specialist. In the Netherlands, the hearing aid dispenser is the only hearing care professional who is allowed to sell hearing aids. The hearing aid dispenser advised the resident about the most suitable hearing aid(s), fit the selected hearing aid(s), taught the resident how to use the hearing aid(s), and supported the resident during the trial period. Residents who already owned hearing aids could consult the hearing aid dispenser for adjustments (refitting or replacement of the earmould and tube) or for new hearing aids (replacement of previously fit hearing aids or extension from monaural to binaural fitting).

Approximately 5 months after the first consultations with the ENT specialist, the audiologist and the ENT specialist held scheduled follow-up consultations with all residents for whom they had provided a hearing aid prescription. At this follow-up visit, the hearing aid trial was evaluated, and the residents had to give their final decision about whether or not they wanted to keep and purchase the hearing aid(s). 


\section{Cost calculation}

We calculated the personnel costs of the audiology assistant, ENT specialist, and audiologist by multiplying the scheduled duration of their activities by their average labour costs which we derived from the Collective Labour Agreement and Dutch manual for costing research (Hakkaart-van Roijen et al., 2010; Nederlandse Vereniging van Ziekenhuizen, 2011). The total travel costs were calculated by multiplying the travel time by the personnel's average labour costs plus a travel distance compensation of $€ 0.20$ per kilometre (Hakkaart-van Roijen et al., 2010).

For the screening (pure-tone audiometry) and additional hearing testing (speech audiometry), the Interacoustics AD 229e audiometer was used. The new price of this audiometer is $€ 3050$. As recommended in the Dutch manual for costing research, we estimated the respective annual costs of depreciation and maintenance to be $10 \%$ and $8 \%$ of the new price (Hakkaart-van Roijen et al., 2010). A year counts about 1540 working hours (Hakkaart-van Roijen et al., 2010). Assuming that the audiometer is in use half of the time, the depreciation and maintenance costs are $€ 0.18$ per 15 minutes of utilisation.

The 2010 unit price of a monaural hearing aid trial was $€ 354$ (Grutters et al., 2008; Statistics Netherlands, 2010). This includes the labour costs of the hearing aid dispenser and the depreciation and maintenance costs of the instruments used. A monaural trial consisted of three consultations with an average duration of 15 to 20 minutes each; a binaural trial consisted of three consultations of approximately 25 to 30 minutes. Because a binaural trial took approximately one and a half time longer than a monaural trial, the unit price of a binaural trial was estimated to be $€ 531$. For adjustment of a previously fit hearing aid (one consultation of approximately 15 minutes), the unit price was estimated to be one-fourth of the unit price of a monaural trial, thus $€ 89$. In 2009, the average price of a purchased hearing aid in the Netherlands was $€ 1079$ (2010 unit price $=€ 1093$ ), including the fitting process, hearing aid, and earmould (Boer\&Croon, 2010; Statistics Netherlands, 2010). The costs of the fitting process were $€ 354$, thus the costs of the hearing aid and earmould were $€ 739$. In 2010, people could obtain a reimbursement of $€ 496.50$ per hearing aid from their health care insurance company.

\section{Analysis}

The effectiveness of the hearing screening and rehabilitation programme was assessed by analysing (1) the proportion of residents who participated in the screening, (2) the proportion of residents with hearing loss who were willing to start rehabilitation, and (3) the rehabilitation success rate. We defined successful rehabilitation as having purchased one or two hearing aids or as having adjusted a previously fit hearing aid. The total programme costs were calculated and the cost distribution was examined. The costs per successfully rehabilitated resident were calculated by dividing the total costs by the number of residents for whom participation in the programme had resulted in successful rehabilitation. 


\section{Results}

\section{Effects}

The eight residential care homes accommodated 705 elderly people (79\% female; interquartile age range $=82-89$ years $)$, of whom $26 \%(\mathrm{~N}=183)$ already owned hearing aids. Figure 1 shows separate programme participation numbers for residents with and without previously fit hearing aids. Overall, 34\% $(\mathrm{N}=243)$ of the residents participated in the screening, $91 \%(\mathrm{~N}=222)$ of whom were screened positive for hearing loss. Of the residents with a positive screen outcome, $56 \%(\mathrm{~N}=125)$ consulted at least one hearing care professional (i.e., ENT specialist, audiologist, or hearing aid dispenser) after the screening. Seventy-three percent $(\mathrm{N}=91)$ of the residents who consulted a professional started rehabilitation in the form of a hearing aid trial $(\mathrm{N}=82$; rehabilitation success rate $=50 \%)$ or adjustment of a previously fit hearing aid $(\mathrm{N}=9$; rehabilitation success rate $=100 \%)$. Hearing aids were purchased by 29 residents without previously fit hearing aids (14 monaural, 15 binaural) and by 12 existing hearing aid owners (10 monaural, 2 binaural). Hearing aid ownership among the residents with hearing loss increased from $28 \%$ at the start of the programme to $33 \%$ at the end.

\section{Costs}

Table 1 gives an overview of the cost calculation. The successful hearing aid trials and the hearing aids were the most costly aspects of the programme, consisting of $64 \%$ of the total programme costs, followed by the negative trials ( $19 \%$ of total costs) and the remaining costs of rehabilitation ( $11 \%$ of total costs). The latter included the costs of additional hearing testing, consultations by the ENT specialist and audiologist, and hearing aid adjustments. Recruitment ( $4 \%$ of total costs) and screening ( $2 \%$ of total costs) were the cheapest aspects of the programme. The health care system paid for two-thirds of the total costs (or two-thirds of the costs per successfully rehabilitated resident); the remaining one-third was paid for by the residents who purchased hearing aids during the programme. The costs were $€ 1896$ per successfully rehabilitated resident. If the programme had been targeted exclusively to residents who already owned hearing aids or exclusively to residents who did not yet own hearing aids, the respective costs would have been $€ 1194$ or $€ 2522$ per successfully rehabilitated resident. 


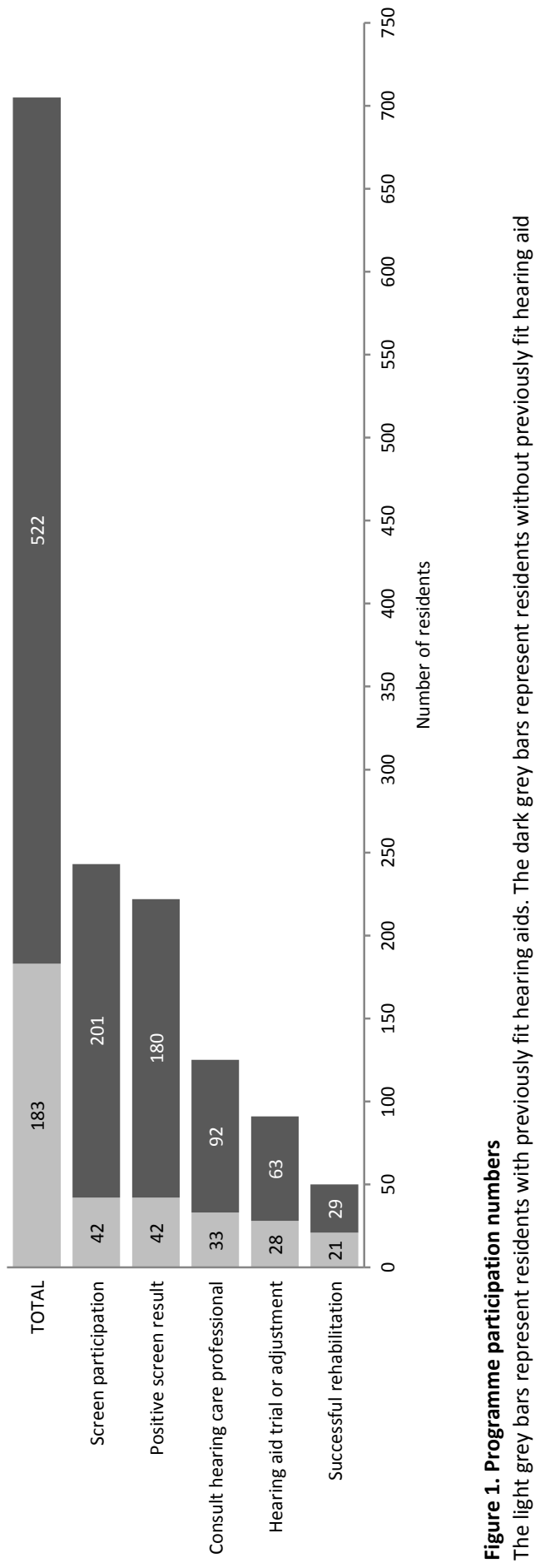


$\stackrel{4}{\square}$

- $+\frac{a}{\Sigma}$
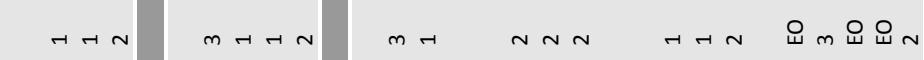

ำ

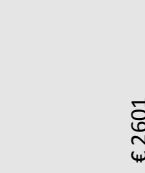

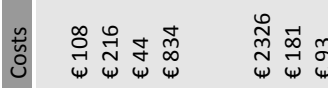

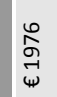

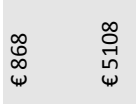

$\underset{\substack{0 \\ \oplus}}{\infty}$

$\underset{\substack{\text { } \\ \Psi}}{\stackrel{w}{N}}$

农

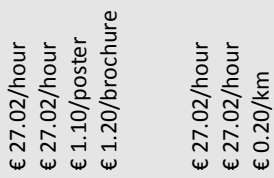

离 言

荡

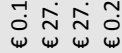

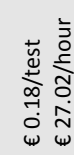

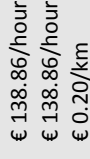

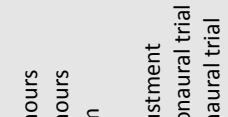

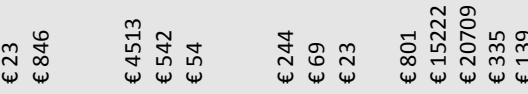

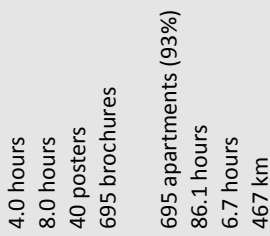

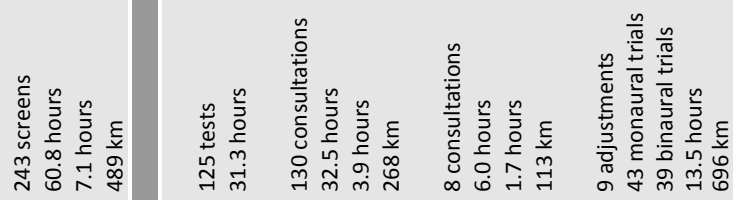

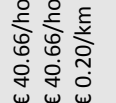

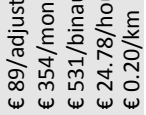

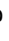

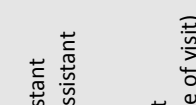

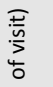

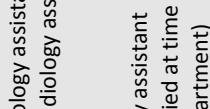

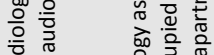

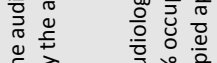

‡ ڤ

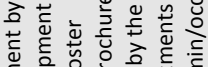

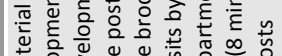

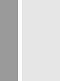

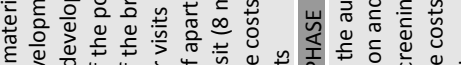

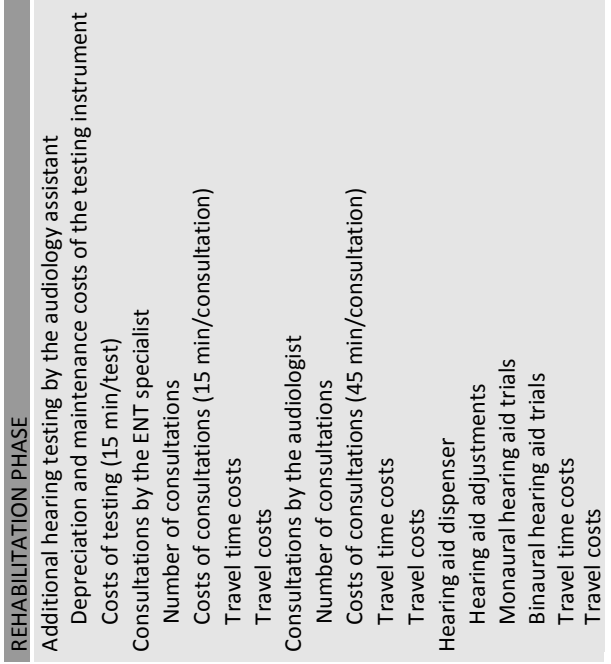




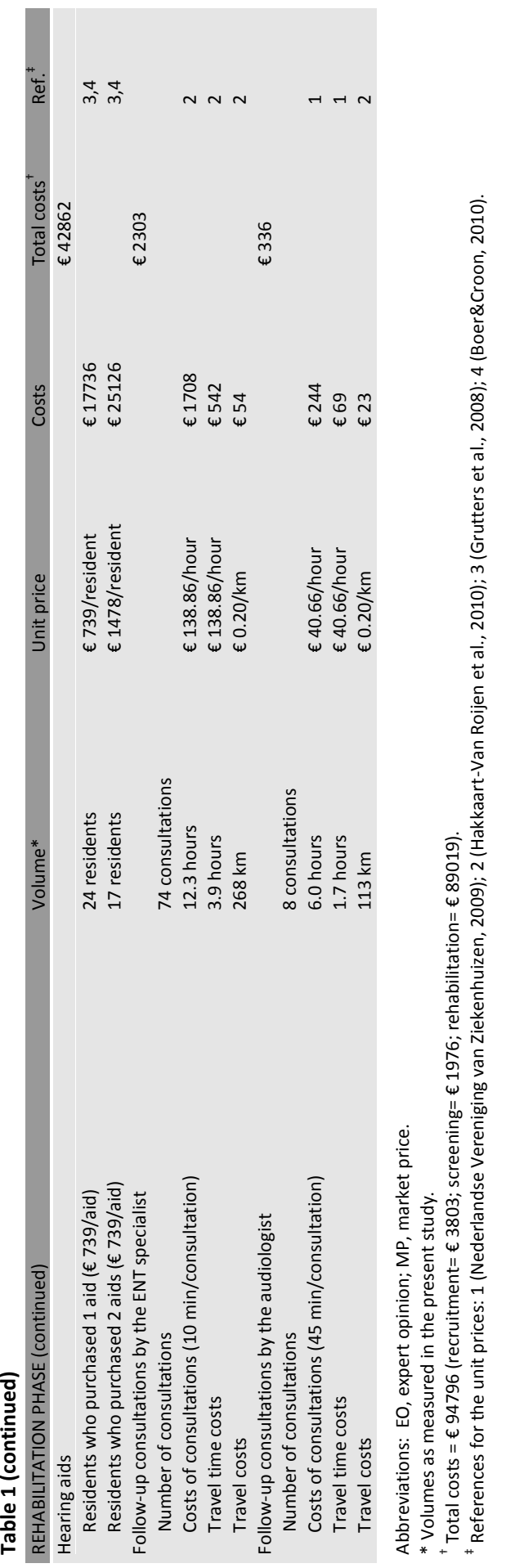




\section{Discussion}

The programme's effectiveness was limited, as hearing aid ownership increased only slightly. Programme participation and rehabilitation uptake are comparable with the results reported by Gussekloo and colleagues (2003). They organised a similar kind of programme in a cohort of 85 -year-old Dutch persons (57\% community dwelling) and found an increase in hearing aid use from $34 \%$ to $50 \%$. Thus, hearing aid uptake among the oldest citizens remains low, even when the accessibility of the hearing care services is optimised. Most residents who were not willing to participate in the programme stated that they were more concerned with attempts to improve other aspects of their functioning, which they perceived as more problematic than their hearing loss.

This study also included residents who already owned a hearing aid; approximately one-fourth of them participated in the screening, and two-thirds of the screen participants started rehabilitation. Thus, the perceived need to see a hearing care professional among these residents was rather high. Apparently, many of the hearing aid owners were not fully satisfied with their hearing aids but had not taken the initiative to return to their hearing aid dispenser. Regular follow-up visits by the hearing aid dispenser to hearing aid owners in residential care homes should therefore be considered.

The costs per rehabilitated resident seem reasonable. However, to draw stronger conclusions, information on long-term hearing aid use and benefit is warranted. It was not feasible to obtain these results in this study, but it is highly encouraged that these outcomes be included in future research on this topic. To decrease the costs per rehabilitated resident, one should focus on decreasing the number of negative hearing aid trials, because they accounted for a substantial part of the total costs without resulting in health gain. Difficulty handling the hearing aid, dependence on others for putting it in and out, and difficulty getting used to it might have caused negative trial outcomes. Offering comprehensive patient and caregiver education and communication training during the trial might decrease the number of negative hearing aid trials. 


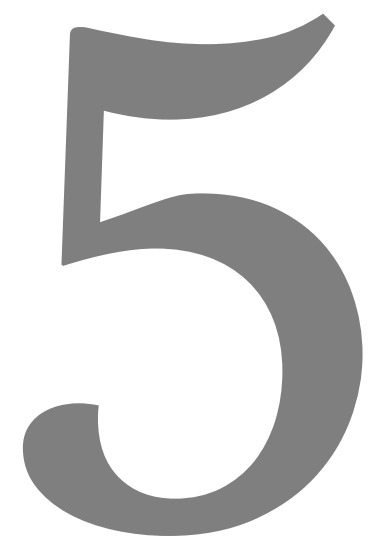

Predictors of hearing acuity: crosssectional and longitudinal analysis

Anouk M Linssen

Martin PJ van Boxtel

Manuela A Joore

Lucien JC Anteunis

Journals of Gerontology: Medical Sciences 2014; 69(6): 759-765 


\begin{abstract}
This study aimed to identify predictors of hearing thresholds (best-ear pure-tone average at 1, 2, and $4 \mathrm{kHz}$ ) and hearing deterioration in order to define potential target groups for hearing screening. We analysed data from the Maastricht Aging Study, a Dutch cohort (aged 24-81 years; $\mathrm{N}=1721$ ) that was observed for 12 years. Mixed model analysis was used to calculate each participant's average hearing threshold deterioration rate during the follow-up period. We built ordinary least square linear regression models to predict the baseline threshold and deterioration rate. Potential predictors included in these models were age, gender, type of occupation, educational level, cardiovascular disease, diabetes, systemic inflammatory disease, hypertension, obesity, waist circumference, smoking, and physical activity level. Poorer baseline thresholds were found to be strongly associated with faster hearing deterioration. Higher age, male gender, manual occupation, and large waist circumference were statistically significantly associated with poorer baseline thresholds and faster deterioration, although the effects of occupation type and waist circumference were small. This study indicates that primarily age and gender must be taken into account when determining the target population for an adult hearing screening programme.
\end{abstract}




\section{Introduction}

Hearing loss is very common in older adults. The prevalence of hearing impairment (expressed as best-ear pure-tone average at $0.5,1,2$, and $4 \mathrm{kHz}\left[\right.$ BEPTA $\left._{0.5-4 \mathrm{kHz}}\right]>25 \mathrm{~dB}$ ) was reported to be $0.6 \%, 2.0 \%, 5.8 \%, 15.0 \%, 31.0 \%$, and $63.1 \%$ for people aged $20-29$ years, 30-39 years, 40-49 years, 50-59 years, 60-69 years, and $\geq 70$ years, respectively (Agrawal et al., 2008; Lin et al., 2011). A high prevalence of hearing impairment has also been associated with male gender (Fransen et al., 2008; Kiely et al., 2012b; Nash et al., 2011), white race (Agrawal et al., 2008; Lin et al., 2011), low educational level (Kiely et al., 2012b; Nash et al., 2011), low household income (Lin et al., 2011), manual labour (Nash et al., 2011), stroke (Kiely et al., 2012b), diabetes (Kiely et al., 2012b), inflammatory bowel disease (Akbayir et al., 2005), rheumatoid arthritis (Takatsu et al., 2005), hypertension (Agrawal et al., 2008; Brant et al., 1996), overweight (Fransen et al., 2008), large waist circumference (Hwang et al., 2009), smoking (Akbayir et al., 2005; Fransen et al., 2008), and low physical activity (Nash et al., 2011), as well as with exposure to sunlight (Michikawa et al., 2013), noise, chemicals, and ototoxic medication (Van Eyken et al., 2007). However, the literature also describes contradictory findings.

Factors associated with hearing loss prevalence are expected to be related to hearing loss incidence and progression as well. Cruickshanks and colleagues (2003) found that age, gender, and type of occupation, but not occupational noise exposure and educational level, were significantly associated with the 5-year incidence of hearing impairment (worst-ear PTA $0.5-4 \mathrm{kHz}>25 \mathrm{~dB}$ ) and that only age was associated with the 5-year progression (hearing threshold deterioration $>5 \mathrm{~dB}$ ). Mitchell and colleagues (2011) reported similar results for the 5 -year incidence of hearing impairment in the best ear $\left(\right.$ BEPTA $_{0.5-4 \mathrm{kHz}}>25 \mathrm{~dB}$ ) and the 5-year progression. Longitudinal studies on the hearing threshold deterioration rate only consistently identified age as a significant predictor (Brant et al., 1996; Davis et al., 1990; Gates \& Cooper, 1991; Karlsmose et al., 2000; Kiely et al., 2012b).

This study examined the association between hearing thresholds (BEPTA ${ }_{1-4} \mathrm{kHz}_{2}$ ) and a large set of potential predictors in a Dutch cohort between 24 and 81 years old that was observed for 12 years. We also longitudinally examined the association between the hearing threshold deterioration rate and a large array of potential predictors. Some of these predictors have not been included in previous comparable studies of this kind (Brant et al., 1996; Davis et al., 1990; Gates \& Cooper, 1991; Karlsmose et al., 2000; Kiely et al., 2012b). Furthermore, the large sample size, the wide age range of the study population, and the long follow-up period distinguish this study from the two earlier European longitudinal studies on hearing deterioration (Davis et al., 1990; Karlsmose et al., 2000). The results of this study could be considered in the development of a targeted screening programme for hearing loss. 


\section{Methods}

\section{Study population}

The total study population consisted of 1823 participants (aged 24-81 years) from the Maastricht Aging Study (MAAS), a longitudinal study on the determinants of cognitive aging, which started in 1993 (Jolles et al., 1995; Van Boxtel et al., 1998). Data on participants aged 49 years and older were collected at four points in time: at baseline $\left(T_{0}\right)$ and at $3\left(T_{3}\right), 6\left(T_{6}\right)$, and 12 years $\left(T_{12}\right)$ after baseline. Data on younger participants were collected at $T_{0}, T_{6}$, and $T_{12}$.

The hearing thresholds of the MAAS participants were initially assessed in a soundproof booth. From January 1994, however, the hearing tests were performed in a quiet room that was not fully soundproof. To overcome eventual bias from this change in measurement setting, we have not used the audiometric data collected before January 1994. Neither did we use the audiometric data of people who reported suffering from Ménière's disease (they have fluctuating hearing acuity, which was judged to be uninteresting for this study).

For logistical reasons, 85 people did not have their hearing tested after January 1 , 1994, and were therefore excluded, as were the 17 people who reported Ménière's disease at their first hearing test. Thus, 1721 MAAS participants remained eligible for participation in the cross-sectional analysis. For the longitudinal analysis, 313 more people were excluded, either because their hearing was tested only once $(n=291)$ or because they reported Ménière's disease at their second hearing test $(n=22)$. Accordingly, 1408 MAAS participants remained eligible for participation in the longitudinal analysis.

\section{Measures}

Pure-tone hearing thresholds were assessed for each ear at frequencies of $0.5,1,2$, and $4 \mathrm{kHz}$ using a screening audiometer (Interacoustics AS7, Denmark) in combination with circumaural headphones. In this study, we used the thresholds at 1,2 , and $4 \mathrm{kHz}$. The thresholds were based on ascending responses using up $5 \mathrm{~dB}$, down $10 \mathrm{~dB}$ steps with $0 \mathrm{~dB}$ as the lowest and $90 \mathrm{~dB}$ as the highest stimulus intensity, which is a variation of the Hughson-Westlake method for audiometric testing (Hughson \& Westlake, 1944). The participants' BEPTA ${ }_{1-4} \mathrm{kHz}$ was calculated at every point in time. Other objectively measured participant characteristics included height, weight, waist circumference, and blood pressure. Individuals with a body mass index of $30 \mathrm{~kg} / \mathrm{m}^{2}$ or more were classified as obese (World Health Organisation, 2008). Participants with a waist circumference more than $102 \mathrm{~cm}$ (men) or $88 \mathrm{~cm}$ (women) were labelled as having a large waist circumference (World Health Organisation, 2008). Participants with a diastolic blood pressure above $90 \mathrm{mmHg}$ were considered to be hypertensive. Furthermore, hypertension was diagnosed whenever the systolic blood pressure was greater than 140 $\mathrm{mmHg}$ (for adults younger than age 60 ) or $160 \mathrm{mmHg}$ (for adults aged 60 or older) (Walma et al., 2003). 
Information on the participants' type of occupation (manual versus intellectual labour), level of education (primary education or lower vocational training versus higher education), presence of cardiovascular disease, presence of diabetes, presence of a systemic inflammatory disease (either rheumatoid arthritis or inflammatory bowel disease), smoking status (current smoker versus ex- or nonsmoker), and physical activity (low versus moderate to high) was obtained by self-report.

\section{Analysis}

Ordinary least square (OLS) linear regression models were made to predict BEPTA $A_{1-4 \mathrm{kHz}}$ at baseline. Predictors included in the models were: age, age ${ }^{2}$, gender, type of occupation, educational level, cardiovascular disease, diabetes, chronic inflammatory disease, hypertension, obesity, waist circumference, smoking, and physical activity level. The strength (B-estimates) and statistical significance ( $p$-values) of the associations between baseline BEPTA ${ }_{1-4} \mathrm{kHz}$ and the predictors (baseline values) were examined in univariate models first, and thereafter in a full multivariate model that included all predictors. Next, we excluded nonsignificant predictors $(p>0.05)$ from the full multivariate model one by one (backward selection). The adjusted $R^{2}\left(R_{\text {adj }}{ }\right.$ ) of the resulting "final" multivariate model and the univariate models were compared with each other. The $R^{2}$ adj reflects the proportion of the total interindividual variance in baseline BEPTA $_{1-4} \mathrm{kHz}$ that is explained by the baseline values of the predictor(s) in the model, with an adjustment for the number of predictors in the model so that an increase in the number of predictors does not necessarily result in an increase in $\mathrm{R}^{2}$ adj.

We started the longitudinal analysis by examining the pattern of hearing threshold deterioration over time. Two linear mixed models were made to determine whether the change in BEPTA $_{1-4 \mathrm{kHz}}$ hearing levels over time followed a linear or an exponential pattern during the 12-year follow-up period. The models included random and fixed effect variance components for the intercept and slope (either time or time ${ }^{2}$ ) with an unstructured covariance matrix. By specifying a model with a random intercept and a random slope, a linear (in case of time) or a quadratic (in case of time ${ }^{2}$ ) regression line was fitted for each individual separately. The goodness of fit of linear mixed models is expressed by the Bayesian Information Criteria (BIC) value, where lower BIC values indicate better model fit. We compared the BIC value for the model with time with the $\mathrm{BIC}$ value for the model with time ${ }^{2}$ to assess whether the actual pattern of BEPTA $1-4 \mathrm{kHz}$ hearing levels over time is best described by a linear or by a quadratic model.

Next, we calculated the average hearing deterioration rate for each individual, based on the regression line that was predicted by the linear mixed model with time. With OLS linear regression, we examined the relationship between baseline BEPTA ${ }_{1-4} \mathrm{kHz}$ and the deterioration rate. Furthermore, OLS linear regression models (univariate models and a full and final multivariate model) were made to predict the individuals' average hearing deterioration rate based on the baseline values of the following nonaudiometric predictors: age, age ${ }^{2}$, gender, type of occupation, educational level, cardiovascular disease, diabetes, chronic inflammatory disease, hypertension, obesity, waist circumference, smoking, and physical activity level. We inspected the predictors' 
B-estimates and $p$-values as well as the proportion of the interindividual variance in hearing deterioration rates explained by the predictor(s) in the model $\left(R^{2}{ }_{\text {adj }}\right)$.

All analyses were done for the total population, and for the young (24-42 years), middle-aged (43-62 years), and older (63-81 years) adults separately.

\section{Results}

\section{Participants}

The baseline characteristics of the participants who were included in the crosssectional $(\mathrm{N}=1721)$ and longitudinal analysis $(\mathrm{N}=1408)$ are given in Table 1 (total population) and Appendix Table A1 (separately per age group). Of the 1408 people who were included in the longitudinal analysis, 411 (29\%) had their hearing tested twice, 701 (50\%) had their hearing tested three times, and $296(21 \%)$ had their hearing tested four times within a follow-up period of 3 to 12 years. For 149 people (11\%), the time span between the first and the last audiometric assessment was 3 years; for 379 people (27\%) it was 6 years; for 109 people (8\%) it was 9 years; and for 771 people $(55 \%)$ it was 12 years. The mean length of follow-up was 9.5 years.

Table 1. Baseline population characteristics

\begin{tabular}{|c|c|c|}
\hline & $\begin{array}{l}\text { Cross-sectional } \\
\text { analysis }(\mathrm{N}=1721)\end{array}$ & $\begin{array}{l}\text { Longitudinal } \\
\text { analysis ( } N=1408 \text { ) }\end{array}$ \\
\hline Age, median (range), in years & $52.7(24.0-84.7)$ & $51.3(24.0-83.7)$ \\
\hline BEPTA, median (IQR), in dB & $11.7(5.0-23.3)$ & $10.0(5.0-21.7)$ \\
\hline BEPTA $>25 \mathrm{~dB}, n(\%)$ & $380(22.1)$ & $269(19.1)$ \\
\hline Male gender, $n(\%)$ & $868(50.4)$ & 731 (51.9) \\
\hline Manual occupation, $n$ (\%) & $832(49.6)$ & $664(48.2)$ \\
\hline Low educational level, $n(\%)$ & 651 (37.9) & $511(36.3)$ \\
\hline Cardiovascular disease, $n(\%)$ & $289(16.8)$ & $207(14.7)$ \\
\hline Diabetes, $n(\%)$ & $70(4.1)$ & $51(3.6)$ \\
\hline Chronic inflammatory disease, $n(\%)$ & $175(10.2)$ & $135(9.6)$ \\
\hline Hypertension, $n(\%)$ & $325(18.9)$ & $258(18.3)$ \\
\hline Obesity, $n(\%)$ & $341(19.8)$ & $263(18.7)$ \\
\hline Large waist circumference, $n(\%)$ & $480(27.9)$ & $369(26.2)$ \\
\hline Smoking, current, $n$ (\%) & $478(27.8)$ & $377(26.8)$ \\
\hline Low physical activity, $n(\%)$ & $773(45.1)$ & $635(45.1)$ \\
\hline
\end{tabular}

\section{Predictors of the baseline hearing threshold (cross-sectional analysis)}

Table 2 shows the final multivariate model for the total population; the univariate models and the full multivariate model can be found in Appendix Table A2. In all models for the total population, age was by far the strongest predictor of baseline BEPTA $_{1-4} \mathrm{kHz}$. We found a positive quadratic relationship between baseline BEPTA ${ }_{1-4} \mathrm{kHz}$ and baseline age (Figure 1). The model with age and age ${ }^{2}$ explained $48.7 \%$ $(95 \% \mathrm{Cl}=45.3-52.1)$ of the total interindividual variance in $\mathrm{BEPTA}_{1-4} \mathrm{kHz}$. Inclusion of other statistical significant predictors - that is, gender, type of occupation, and waist 
circumference - raised the explained variance to $50.9 \%(95 \% \mathrm{Cl}=47.5-54.2$; final multivariate model). Gender was the second strongest predictor of baseline BEPTA ${ }_{1-4}$ $\mathrm{kHz}$ in the final multivariate model with men having $4.2 \mathrm{~dB}(95 \% \mathrm{Cl}=3.2-5.1)$ poorer hearing thresholds than women.

Table 2 also shows the results of the final OLS linear regression models for the three age groups separately. Age was still the strongest predictor of baseline hearing thresholds but the proportion of variance in BEPTA $_{1-4 \mathrm{kHz}}$ explained by age was low: $8.5 \%(95 \% \mathrm{Cl}=4.1-12.9)$ in young adults, $9.9 \%(95 \% \mathrm{Cl}=5.5-14.3)$ in middle-aged adults, and $9.4 \%(95 \% \mathrm{Cl}=4.7-14.1)$ in older adults. Inclusion of other statistical significant predictors raised the explained variance to $10.8 \%(95 \% \mathrm{Cl}=6.0-15.6)$ in young adults, $15.2 \%(95 \% \mathrm{Cl}=10.0-20.4)$ in middle-aged adults, and $18.4 \%(95 \% \mathrm{Cl}=12.3-24.5)$ in older adults. Gender was the second strongest predictor of baseline BEPTA ${ }_{1-4} \mathrm{kHz}$ in the final multivariate model for middle-aged and older adults, but we did not find a gender effect in the youngest age group. Adjusted for all other significant predictors, middleaged and older men had, respectively, $4.4 \mathrm{~dB}(95 \% \mathrm{Cl}=2.8-5.9)$ and $7.9 \mathrm{~dB}(95 \% \mathrm{Cl}=5.6-$ 10.2) poorer hearing thresholds than middle-aged and older women. The hearing thresholds of middle-aged and older men were comparable to the hearing thresholds of women who were respectively 8.4 years $(95 \% \mathrm{Cl}=5.5-11.3)$ or 9.1 years $(95 \% \mathrm{Cl}=6.5-$ 11.8) older. A third strong predictor was type of occupation, with older adults with manual labour having $4.8 \mathrm{~dB}(95 \% \mathrm{Cl}=2.5-7.2)$ poorer hearing thresholds than older adults with intellectual labour.

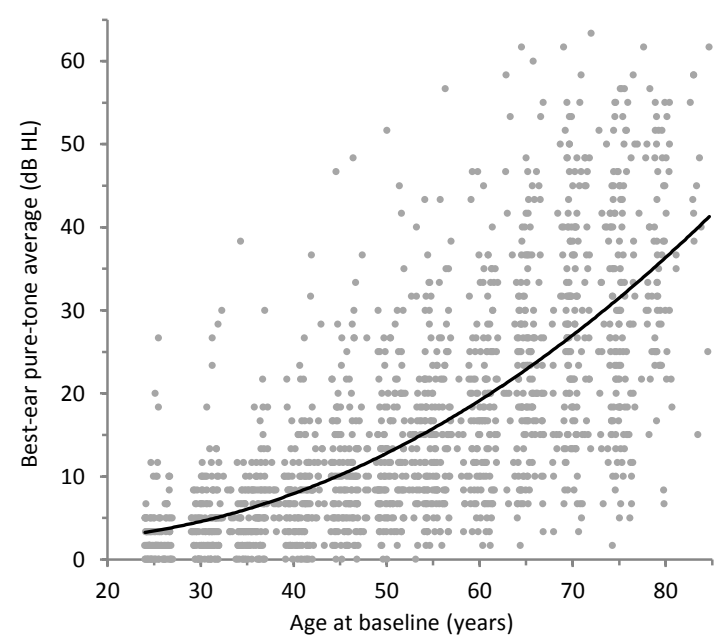

Figure 1. The relationship between the best-ear pure-tone average $(1,2$, and $4 \mathrm{kHz})$ and age at baseline $(\mathrm{N}=1721)$ 


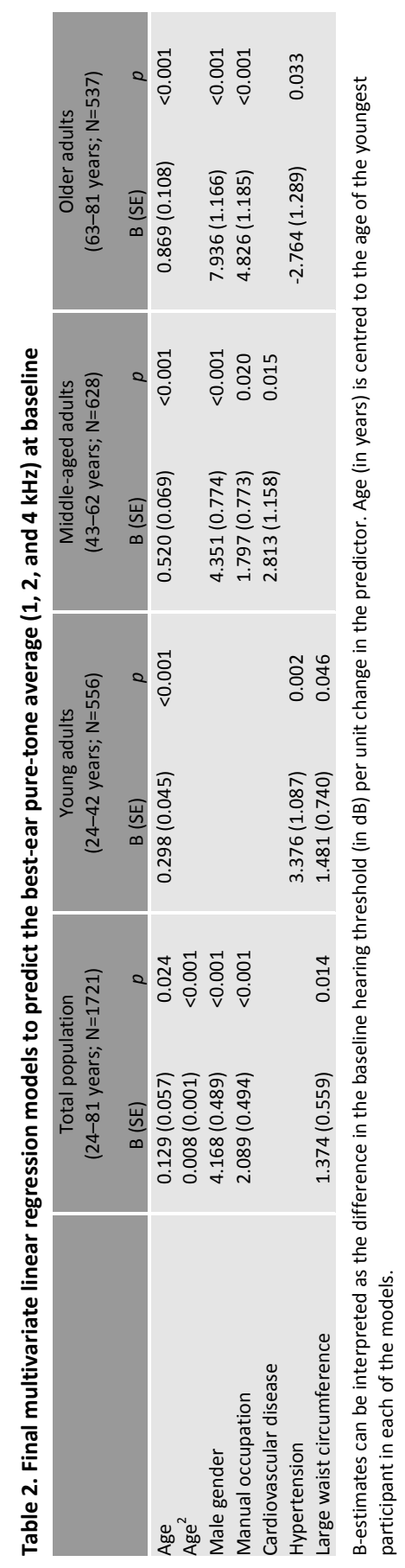




\section{Predictors of the hearing threshold deterioration rate (longitudinal analysis)}

\section{Pattern of hearing deterioration}

The $\mathrm{BIC}$ value for the model with time (BIC=28749) was lower than the $\mathrm{BIC}$ value for the model with time ${ }^{2}(\mathrm{BIC}=29224)$, which means that the pattern of hearing deterioration over time is better represented by a linear function than by a quadratic function. Thus, the study participants' BEPTA $A_{1-4} \mathrm{kHz}_{\mathrm{z}}$ changed at a constant rate during the 12 -year follow-up period. The overall median hearing deterioration rate was $7.3 \mathrm{~dB} /$ decade (interquartile range $[I Q R]=5.3-10.9)$. For young adults, it was $5.1 \mathrm{~dB} /$ decade (IQR=4.26.1); for middle-aged adults, it was $7.6 \mathrm{~dB} /$ decade (IQR=6.0-10.0); and for older adults, it was $12.3 \mathrm{~dB} /$ decade (IQR=9.5-14.6).

\section{Deterioration rate in relation to the baseline hearing threshold}

The hearing deterioration rate was linearly related to baseline BEPTA ${ }_{1-4} \mathrm{kHz}$ (Figure 2). In a univariate model, baseline BEPTA ${ }_{1-4} \mathrm{kHz}$ explained $79.5 \%(95 \% \mathrm{Cl}=77.6-81.4)$ of the total interindividual variance in the hearing deterioration rate. The proportion of variance explained by baseline $\mathrm{BEPTA}_{1-4} \mathrm{kHz}$ was $45.8 \%(95 \% \mathrm{Cl}=39.2-52.4)$ in young adults, $60.7 \%(95 \% \mathrm{Cl}=55.6-65.8)$ in middle-aged adults, and $75.5 \%(95 \% \mathrm{Cl}=71.2-79.8)$ in older adults.

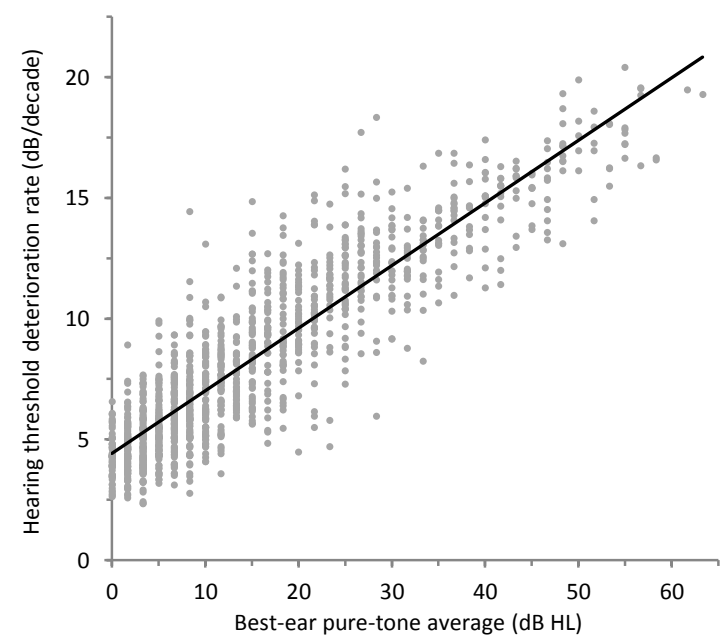

Figure 2. The relationship between the hearing threshold deterioration rate and the best-ear pure-tone average $(1,2$, and $4 \mathrm{kHz})$ at baseline $(\mathrm{N}=1408)$ 


\section{Deterioration rate in relation to nonaudiometric factors}

Table 3 shows the final multivariate model for the total population; the univariate models and the full multivariate model can be found in Appendix Table A3. In all models for the total population, age was by far the strongest nonaudiometric predictor of the hearing threshold deterioration rate. The hearing deterioration rate was quadratically related to baseline age (Figure 3 ). The model with age and age ${ }^{2}$ explained $54.8 \%(95 \% \mathrm{Cl}=51.3-58.3)$ of the total interindividual variance in the hearing deterioration rate. Inclusion of other statistical significant nonaudiometric predictors - that is, gender, type of occupation, and waist circumference - raised the explained variance to $56.6 \%(95 \% \mathrm{Cl}=53.2-60.0$; final multivariate model). Gender was the second strongest nonaudiometric predictor of the deterioration rate in the final multivariate model with men having a $1.1 \mathrm{~dB} /$ decade $(95 \% \mathrm{Cl}=0.8-1.4)$ faster deterioration than women.

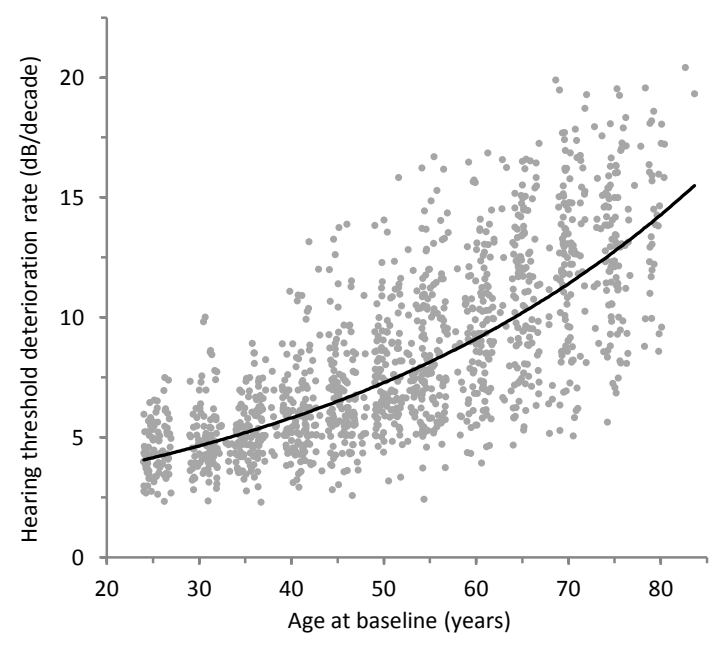

Figure 3. The relationship between the hearing threshold deterioration rate and age at baseline $(\mathrm{N}=1408)$ 


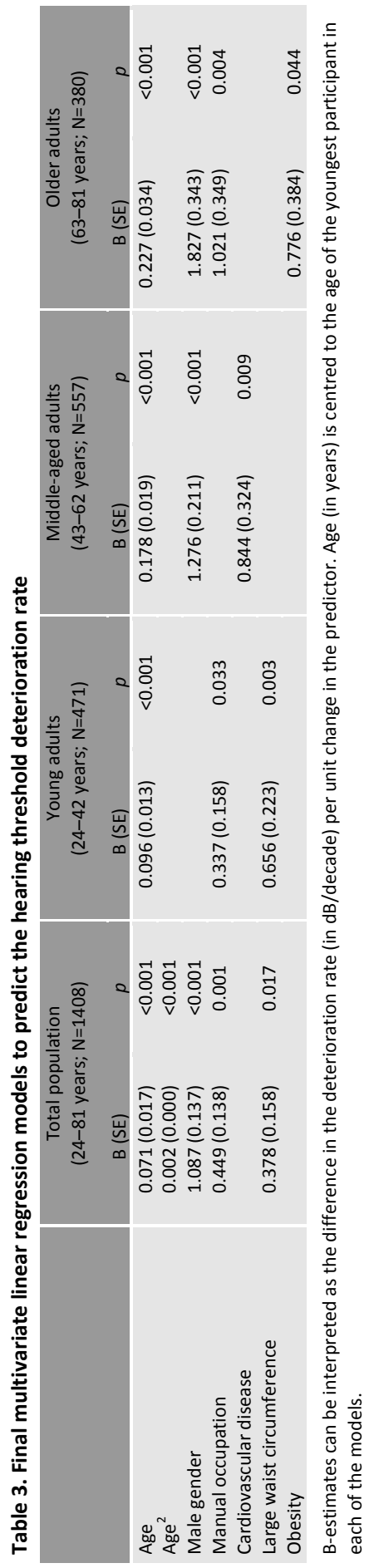


Table 3 also shows the results of the final OLS linear regression models for the three age groups separately. Age was still the strongest nonaudiometric predictor of the deterioration rate but the proportion of variance explained by age was low: $9.8 \%$ $(95 \% \mathrm{Cl}=4.7-14.9)$ in young adults, $14.5 \%(95 \% \mathrm{Cl}=9.1-19.9)$ in middle-aged adults, and $8.9 \%(95 \% \mathrm{Cl}=3.5-14.3)$ in older adults. Inclusion of other statistical significant nonaudiometric predictors raised the explained variance to $12.6 \%(95 \% \mathrm{Cl}=7.0-18.2)$ in young adults, $20.7 \%(95 \% \mathrm{Cl}=14.8-26.6)$ in middle-aged adults, and $16.4 \%(95 \% \mathrm{Cl}=9.5-$ 23.3) in older adults. Gender was the second strongest nonaudiometric predictor of the hearing deterioration rate in the final multivariate model for middle-aged and older adults but not for young adults. Adjusted for all significant nonaudiometric predictors, the deterioration rate was, respectively, $1.3 \mathrm{~dB} /$ decade $(95 \% \mathrm{Cl}=0.9-1.7)$ and $1.8 \mathrm{~dB} /$ decade $(95 \% \mathrm{Cl}=1.2-2.5)$ higher in middle-aged and older men than in middle-aged and older women.

\section{Discussion}

Consistent with other studies, we found that higher age and male gender were significantly associated with poorer baseline hearing levels (Agrawal et al., 2008; Fransen et al., 2008; Kiely et al., 2012b; Lin et al., 2011; Nash et al., 2011). The positive quadratic relationship we found between age and the deterioration rate has also been described in earlier studies (Kiely et al., 2012b; Pearson et al., 1995). Age explained $8.9 \%$ of the interindividual variance in the deterioration rate in adults $\geq 63$ years in this study, which is comparable to the $9 \%$ described by Gates and Cooper (1991) and by Viljanen and colleagues (2007) for adults aged $\geq 58$ years.

Poorer hearing at baseline (BEPTA ${ }_{1-4} \mathrm{kHz}$ ) was significantly associated with faster hearing deterioration in the subsequent 3-12 years. The baseline hearing levels explained $79.5 \%$ of the interindividual variance in the deterioration rate. To our knowledge, only two earlier studies have examined the association between baseline pure-tone average thresholds and the deterioration rate (Davis et al., 1990; Gates \& Cooper, 1991). No significant association with the deterioration rate was found for PTA P.5- $_{0.5 z}$ (Davis et al., 1990) and PTA $0.5-2 \mathrm{kHz}$ (Gates \& Cooper, 1991), but poorer baseline PTA $4-8 \mathrm{kHz}$ was found to be related to slower deterioration (Gates \& Cooper, 1991). In these two studies, the analyses were performed for the left and right ear separately. The mean age of our population was lower, our study population was much larger, and our follow-up period was twice as long. These differences may explain the discrepancy in results between the previous and current studies. Poorer baseline hearing levels (in comparison to age-matched peers) may reflect a high genetic susceptibility to hearing loss, which would explain the faster deterioration we found in adults with poorer baseline hearing levels. Cross-sectional twin studies recently revealed that interindividual differences in hearing levels are primarily attributed to genetic variation: for example, Viljanen and colleagues (2007) found that genetic effects accounted for $75 \%$ $(95 \% \mathrm{Cl}=67-81)$ of the total variance in $\mathrm{BEPTA}_{0.5-4} \mathrm{kHz}$ for older women and Wingfield 
and colleagues (2007) found that genetic effects explained $65 \%-66 \%(95 \% \mathrm{Cl}=45-74)$ of the total variance in both BEPTA $_{0.5-2 \mathrm{kHz}}$ and BEPTA $4-8 \mathrm{kHz}$ for older men.

We found a great overlap between the predictors of baseline BEPTA $\mathrm{A}_{1-4 \mathrm{kHz}}$ and the predictors of the hearing deterioration rate (even $100 \%$ agreement in the nonstratified models), which is not surprising, given the strong relationship between baseline BEPTA $_{1-4 \mathrm{kHz}}$ and the deterioration rate. Gender, type of occupation, cardiovascular disease, waist circumference, and obesity were related to the hearing deterioration rate in at least one of the age groups, but their effect was consistently small (maximum $1.8 \mathrm{~dB}$ /decade). This is the first longitudinal study to examine the relationship between the hearing threshold deterioration rate and chronic inflammatory disease, obesity, waist circumference, and physical activity level. Studies on the relationship between cardiovascular disease, smoking, and diabetes at baseline have consistently reported that these factors are not related to the hearing threshold deterioration rate (Brant et al., 1996; Davis et al., 1990; Karlsmose et al., 2000; Kiely et al., 2012b). Studies on the deterioration rate in BEPTA $_{0.5-4 \mathrm{kHz}}$ have not detected a gender difference (Davis et al., 1990; Kiely et al., 2012b; Mitchell et al., 2011), although gender differences in the single frequency deterioration rate have been reported (Lee et al., 2005; Pearson et al., 1995; Wiley et al., 2008). Previous longitudinal studies also found inconsistent results for type of occupation, educational level, and hypertension (Brant et al., 1996; Cruickshanks et al., 2003; Davis et al., 1990; Karlsmose et al., 2000; Kiely et al., 2012b; Mitchell et al., 2011).

This study has several limitations. First, the audiometric measurements did not take place in a fully soundproof booth. Consequently, ambient noise - which is usually low frequent noise - might have biased the hearing thresholds at the lower frequencies. For this reason, we excluded the $0.5 \mathrm{kHz}$ measurement, but we cannot rule out that the $1 \mathrm{kHz}$ measurement was biased for some participants. Another limitation is the ethnicity of the participants, who were mainly white Caucasian. This limits the generalizability of the results because hearing thresholds are known to differ between races (Agrawal et al., 2008; Lin et al., 2011). A third study limitation is that we were unable to include information about noise exposure in the model. Cross-sectional studies have consistently reported an association between noise exposure and hearing loss (Van Eyken et al., 2007), but it is difficult to accurately estimate noise exposure (e.g. exposure time, frequency, noise intensity, use of ear protection) and to capture it in one or two parameters that can be used in a prediction model. However, previous longitudinal studies have not found a significant relationship between noise exposure and the hearing threshold deterioration rate (Davis et al., 1990; Karlsmose et al., 2000; Lee et al., 2005); neither did noise exposure confound the effects of other predictors (Kiely et al., 2012b). A final study limitation is that we did not collect information on ototoxic medication use, exposure to ototoxic chemicals, or a family history of hearing loss.

We chose to use the best-ear hearing thresholds instead of the worst-ear hearing thresholds because best-ear hearing thresholds were thought to be more closely related to hearing disability, adherence to screening advice, and hearing aid use. However, future studies that are aimed at the causal relationships between environ- 
mental factors and hearing loss (e.g. in order to develop interventions to prevent hearing impairment and further deterioration) are advised to use the worst-ear hearing thresholds, because those have been reported to be more influenced by environmental factors than the best-ear hearing thresholds (Wingfield et al., 2007).

In conclusion, our results indicate that primarily age and gender must be taken into account when determining the target population for an adult hearing screening programme. 


\section{Appendix}

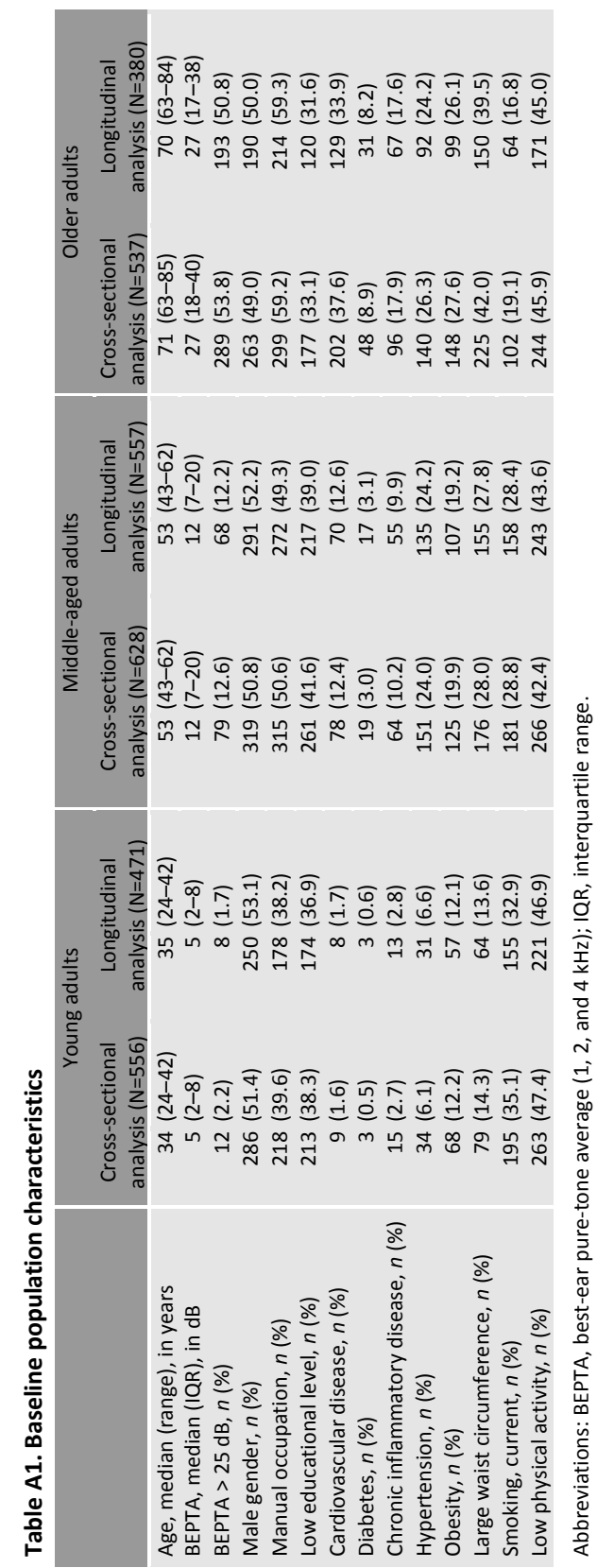


Table A2. Linear regression models to predict the best-ear pure-tone average $(1,2$, and $4 \mathrm{kHz})$ at baseline ( $N=1721)$

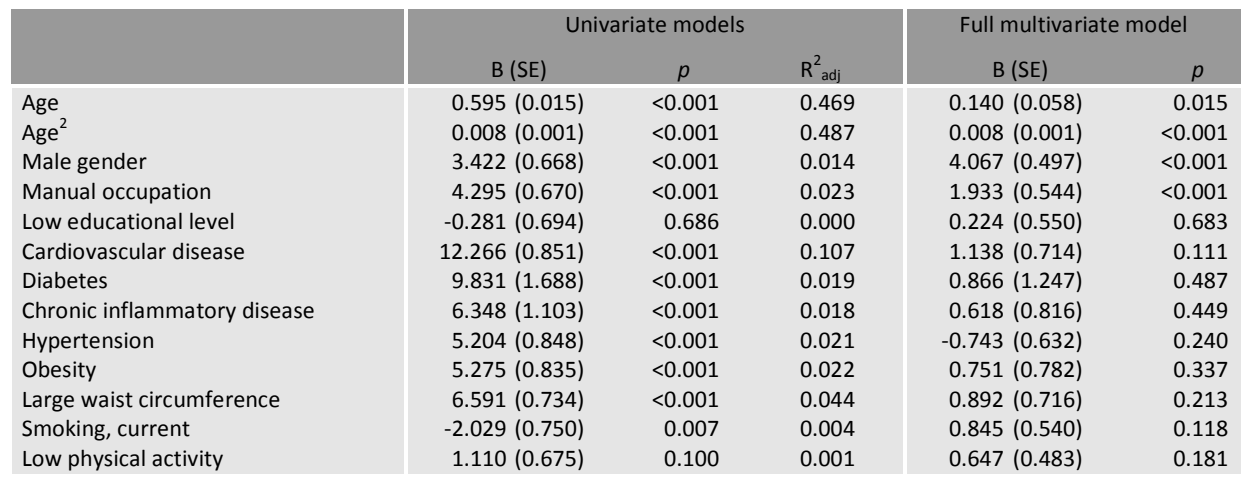

B-estimates can be interpret as the difference in the baseline hearing threshold (in $\mathrm{dB}$ ) per unit change in the predictor. Age (in years) is centred to the age of the youngest participant in the models (i.e. 24 years). The univariate model with age ${ }^{2}$ also includes age.

Table A3. Linear regression models to predict the hearing threshold deterioration rate in $\mathrm{dB} / \mathrm{decade}$ $(\mathrm{N}=1408)$

\begin{tabular}{|c|c|c|c|c|c|}
\hline & \multicolumn{3}{|c|}{ Univariate models } & \multicolumn{2}{|c|}{ Full multivariate model } \\
\hline & $B(S E)$ & $p$ & $R_{\text {adj }}^{2}$ & $B(S E)$ & $p$ \\
\hline Age & $0.180(0.004)$ & $<0.001$ & 0.535 & $0.072(0.017)$ & $<0.001$ \\
\hline $\mathrm{Age}^{2}$ & $0.002(0.0003)$ & $<0.001$ & 0.548 & $0.002(0.0003)$ & $<0.001$ \\
\hline Male gender & $0.824(0.200)$ & $<0.001$ & 0.011 & $1.062(0.140)$ & $<0.001$ \\
\hline Manual occupation & 1.160 (0.199) & $<0.001$ & 0.023 & $0.450(0.152)$ & 0.003 \\
\hline Low educational level & $-0.167(0.209)$ & 0.423 & 0.000 & $-0.068(0.155)$ & 0.663 \\
\hline Cardiovascular disease & $3.223(0.270)$ & $<0.001$ & 0.091 & $0.249(0.205)$ & 0.224 \\
\hline Diabetes & $2.720(0.532)$ & $<0.001$ & 0.018 & $0.089(0.366)$ & 0.807 \\
\hline Chronic inflammatory disease & $1.767(0.338)$ & $<0.001$ & 0.018 & $-0.085(0.233)$ & 0.715 \\
\hline Hypertension & $1.375(0.257)$ & $<0.001$ & 0.019 & $-0.146(0.178)$ & 0.413 \\
\hline Obesity & $1.342(0.255)$ & $<0.001$ & 0.019 & $0.100(0.223)$ & 0.654 \\
\hline Large waist circumference & $1.809(0.223)$ & $<0.001$ & 0.044 & $0.311(0.204)$ & 0.127 \\
\hline Smoking, current & $-0.734(0.226)$ & 0.001 & 0.007 & $0.164(0.153)$ & 0.285 \\
\hline Low physical activity & $0.235(0.202)$ & 0.243 & 0.000 & $0.203(0.136)$ & 0.135 \\
\hline
\end{tabular}

B-estimates can be interpret as the difference in the deterioration rate (in $\mathrm{dB} / \mathrm{decade}$ ) per unit change in the predictor. Age (in years) is centred to the age of the youngest participant in the models (i.e. 24 years). The univariate model with age ${ }^{2}$ also includes age. 


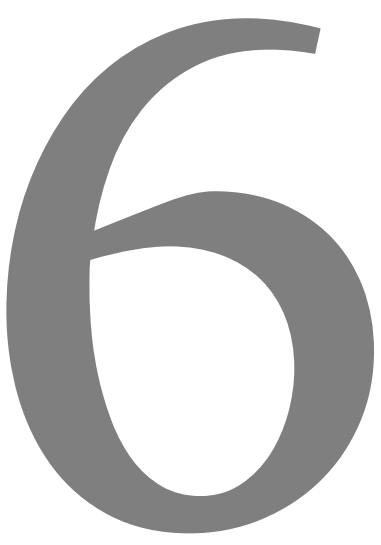

The cost-effectiveness of different hearing screening strategies for 50 - to 70-year-old adults: a Markov model

Anouk M Linssen Lucien JC Anteunis Manuela A Joore

Submitted 


\begin{abstract}
This study aimed to assess the cost-effectiveness of screening 50- to 70-year-old adults for hearing loss in the Netherlands. The costs per quality-adjusted life year (QALY) of current practice (no screening) and 76 adult hearing screening strategies were analysed using a Markov model with cohort simulation for the year 2011. The screening strategies varied in the age at first screening (either 50,55, 60, 65, or 70 years), the number of repeated screenings (up to five repetitions), the time interval between repeated screenings (either 5 or 10 years), and the type of screening which was either telephone screening, internet screening, screening at the practice of the general practitioner (GP) with a hand-held screening device, or screening at the GP practice with a standard audiometer. Screening was deemed to be cost-effective if the costs were less than $€ 20000$ per QALY. We found that all screening strategies were costeffective compared to current practice. Screening at the GP practice was generally more costly but less effective than telephone or internet screening. The internet screening strategies were slightly better than the telephone screening strategies. Internet screening at age 50, repeated at ages 55, 60, 65, and 70 was the most costeffective strategy, costing $€ 3699$ per QALY. At a threshold of $€ 20000$ per QALY this strategy was with $100 \%$ certainty cost-effective compared to current practice and with $69 \%$ certainty the most cost-effective strategy among all strategy. This strategy might be considered for nationwide implementation.
\end{abstract}




\section{Introduction}

Untreated hearing loss has a variety of negative consequences, including social isolation, loneliness, psychosocial distress, anxiety, and depression (Gopinath et al., 2009; Monzani et al., 2008; Nachtegaal et al., 2009). Hearing aid fitting can increase the quality of life of people with a hearing loss (Chia et al., 2007; Chisolm et al., 2007; Vuorialho et al., 2006). It is a cost-effective intervention compared to no rehabilitation (Chao \& Chen, 2008; Joore et al., 2003a). Nevertheless, adults generally postpone seeking help until five to ten years after the onset of their hearing loss (Davis et al., 2007; Leegwater \& Lammerts van Bueren, 2005; Meister et al., 2008). People mainly postpone seeking help because they are unaware or in denial of the hearing loss and believe they can still manage without hearing aids (Van Thiel, 2010).

Seeking help at an early stage is important because adults who start using hearing aids early - that is, at a relatively young age or when their hearing loss is still relatively mild - do not only have more years with benefit ahead, but also have greater benefit from their hearing aids during later life than adults who started using hearing aids late (Davis et al., 2007). This can be explained by the fact that older adults have more difficulty adapting to hearing aids and learning to use new technology because of poorer cognitive performance and poorer learning ability (Humes, 2007; Janacsek et al., 2012). Moreover, people with poorer cognitive performance often have difficulty in formulating their needs during a hearing aid trial, which can result in a suboptimal hearing aid fit (Lunner, 2003).

Adult hearing screening is thought to motivate people to seek help earlier. Studies in the United Kingdom showed that screening can triple hearing aid ownership among middle-aged adults (Davis et al., 1992; Stephens et al., 1990). The only randomised controlled trial to the effectiveness of screening so far, showed that screening with an objective measurement instrument can almost double the 1-year incidence of hearing aid use (Yueh et al., 2010). Adult hearing screening is found to be a cost-effective intervention to increase hearing aid use and quality of life in comparison to no screening (Liu et al., 2011; Morris et al., 2013). Screening with an objective measurement instrument outperformed screening with a subjective measurement instrument (questionnaire) and screening with an objective and subjective instrument combined (Liu et al., 2011; Morris et al., 2013). Our study is the first that assessed the costs and the effects on quality of life of telephone and internet screening. The costs and effects were compared to those of screening with a hand-held screening device (like Liu and colleagues), screening with an audiometer (like Morris and colleagues), and current practice in the Netherlands (no nationwide adult hearing screening programme). 


\section{Methods}

\section{Model structure}

A Markov model was constructed to evaluate the cost-effectiveness of adult hearing screening from a healthcare perspective. All costs were in Euros ( $€ 1.00$ is US $\$ 1.38$ and $f 0.87$, average 2011 conversion rates). The effectiveness measure in the model was the quality-adjusted life year (QALY) which is a combined measure of healthrelated quality of life and duration of life. The model allowed simulating the lifetime course of events in 50-year-old adults who do not own hearing aids. Possible events included hearing deterioration, hearing aid uptake, hearing aid replacement, hearing aid discard, and dying. The health states of the model were based on hearing loss severity and hearing aid use (Figure 1).

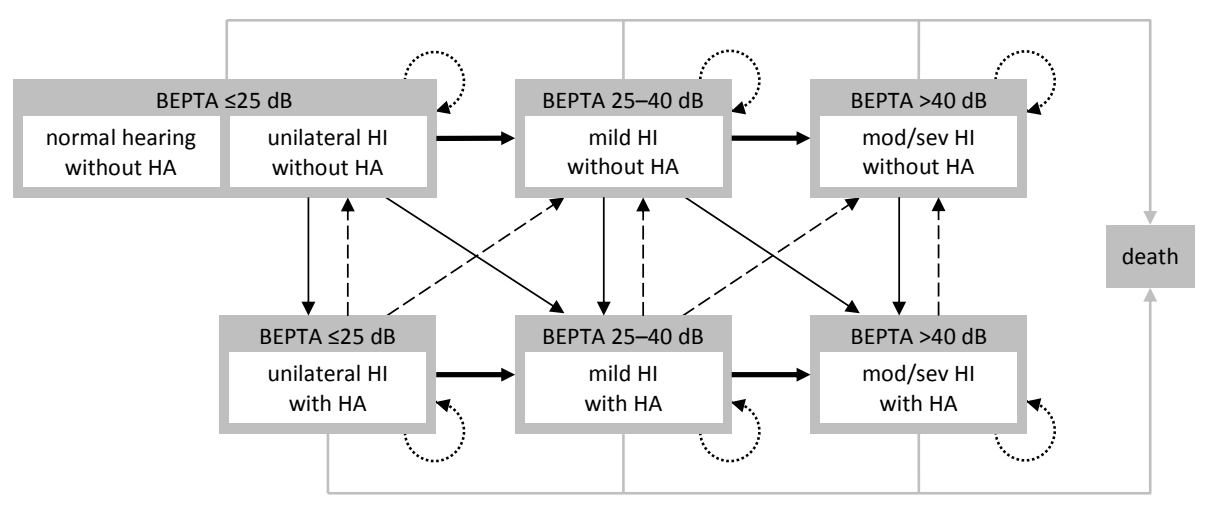

Figure 1. General structure of the Markov model

The solid and dashed lines represent the transitions to other health states by hearing deterioration (bold black lines), hearing aid uptake (thin black lines), hearing aid discard (dashed lines), and dying (grey lines). Screening is offered to people without hearing aids and impacts only the probability of hearing aid uptake. BEPTA, best-ear pure-tone average $(0.5,1,2$, and $4 \mathrm{kHz})$; $\mathrm{HA}$, hearing aid; $\mathrm{HI}$, hearing impairment.

For hearing loss severity, the classification of the World Health Organisation was used with a hearing threshold (pure-tone average at $0.5,1,2$, and $4 \mathrm{kHz}$ ) between 25 to 40 $\mathrm{dB}$ in the best ear indicating mild hearing impairment and a hearing threshold of more than $40 \mathrm{~dB}$ in the best ear indicating moderate to severe hearing impairment. $A$ hearing threshold of $25 \mathrm{~dB}$ or less in the best ear indicated either normal hearing or unilateral hearing impairment. With unilateral hearing impairment we refer to a hearing threshold of $25 \mathrm{~dB}$ or less in the best ear, a hearing threshold of more than 25 $\mathrm{dB}$ in the worst ear, and an interaural difference of either $10 \mathrm{~dB}$ or more at three frequencies, $15 \mathrm{~dB}$ or more at two frequencies, or $20 \mathrm{~dB}$ or more at one frequency. Approximately $27 \%$ of the people with a hearing threshold of $25 \mathrm{~dB}$ or less in the best ear have unilateral hearing impairment (Duijvestijn et al., 2003). We assumed that 
among adults with a hearing threshold of $25 \mathrm{~dB}$ or less in the best ear only adults with unilateral hearing impairment might take up hearing aids. However, we did not consider normal hearing and unilateral hearing impairment as separate health states in our model because data on the annual probability to develop mild hearing impairment for these two groups separately were not available.

The model simulated current practice (no screening) and four types of nationwide screening:

- Telephone screening: the target population is invited by letter to do the National Hearing Test by telephone. The National Hearing Test is a fully automatic adaptive speech-in-noise test that uses digit-triplets as speech material. It was developed and validated by Smits and colleagues (2004). There are three possible outcomes: good, insufficient, and poor hearing. People with insufficient or poor hearing are considered screen-positive. Since only one ear is tested (usually the participant's best ear), the test is unable to detect unilateral hearing impairment.

- Internet screening: the target population is invited by letter to do the National Hearing Test by internet (www.hoortest.nl). The test was developed by Smits and colleagues (2006) and validated by Leensen and colleagues (2011). It is greatly similar to the telephone test, except for the fact that the internet test presents the signals to both ears simultaneously. The internet test will not detect unilateral hearing impairment.

- HearCheck screening: members from the target population who visit the practice of the general practitioner (GP), for whatever reason, are offered a hearing screening test while waiting for a consultation with their GP. The GP assistant performs the screening using the Siemens HearCheck Navigator. The Siemens HearCheck Navigator is a hand-held screening device that emits five tones: $375 \mathrm{~Hz}(35 \mathrm{~dB}), 1000 \mathrm{~Hz}(55 \mathrm{~dB}$ and $35 \mathrm{~dB})$, and $3000 \mathrm{~Hz}(75 \mathrm{~dB}$ and $35 \mathrm{~dB})$. Depending on which tones are heard, the test will inform the participants whether their hearing in the tested ear is good, insufficient or poor. Both ears are tested separately, therefore unilateral hearing impairment can be detected. People with insufficient or poor hearing in one or both ears are considered screen-positive. The GP discusses the outcome with everyone who screened positive.

- Audiometric screening: members from the target population who visit the GP practice, for whatever reason, are offered a hearing screening test while waiting for a consultation with their GP. The GP assistant performs standard pure-tone audiometry. As this is the gold standard, everyone with hearing impairment is screen-positive and everyone with normal hearing is screen-negative. The GP discusses the outcome with everyone who screened positive.

In total, 76 different screening strategies were included in the economic evaluation. The strategies were varied in the type of screening, the age at first screening (either $50,55,60,65$, or 70 years), the number of repeated screenings (up to five repetitions), and the time interval between repeated screenings (either 5 or 10 years). The model 
was constructed using Microsoft Excel 2010 with customised macros and had a lifetime time horizon with a cycle length of 1 year.

\section{Model input}

In the paragraphs below we briefly describe which parameters were accounted for in the model. An elaborate description of our considerations, calculations, and assumptions with regard to the parameter estimates is given in the appendix. Appendix Table A1 lists the model parameter estimates used to calculate the costs and effects of hearing care in current practice, including base case parameter estimates, distributions, and references to data sources. Appendix Table A2 lists the additional model input for the screening specific parameters.

\section{Transition probabilities}

At model entrance, the cohort comprised adults without hearing aids who were distributed over the three possible starting states based on the prevalence of uncorrected mild and moderate to severe hearing impairment in the general population (Mitchell et al., 2011). Hearing deterioration was modelled using estimates of the one-year incidence of mild and moderate to severe hearing impairment that were, like the prevalence data, derived from the Blue Mountains Hearing Study (Mitchell et al., 2011). We used age and gender specific annual mortality figures from the Dutch life tables (Statistics Netherlands, 2011). The probability of hearing aid discard was set at $6 \%$ in all strategies (Van den Brink et al., 1996). We modelled that screening would be offered exclusively to people without hearing aids and that it would only influence the probability of hearing aid uptake (thin black arrows in Figure 1). The probability of hearing aid uptake was modelled separately for current practice and for each screening strategy as a function of the following parameters: the probability of screen participation, the validity of the screening test, the probability of help-seeking (first step), the probability that a help-seeker continues help-seeking, the probability that someone who continued help-seeking starts a hearing aid trial, and the probability that the trial is ended successfully and thus resulted in hearing aid purchase. The screening strategies differed in the probability of screen participation, the validity of the screening test, the probability of help-seeking, and the probability of continued help-seeking. Estimates of these screening specific probabilities and the sources on which they were based can be found in Appendix Table A2. The probability that someone who continued help-seeking starts a hearing aid trial and the probability of trial success were assumed not to be influenced by screening. For adults who did not participate in screening, we used the probabilities of help-seeking and continued help-seeking from current practice.

In the Netherlands, the first step in help-seeking for hearing impairment is a consultation with either the GP or the hearing aid dispenser. Continued help-seeking may include a consultation with the ear, nose, and throat (ENT) specialist, a consultation at the Audiological Centre, and/or an intake meeting with the hearing aid dispenser (see Appendix Figure A1 for a schematic overview of possible paths in 
continued help-seeking). The ENT specialist clinically examines the ear, removes ear wax, diagnoses ear pathology, performs hearing tests, informs the help-seeker about treatment possibilities, and prescribes hearing aids to those who are willing to try them. Furthermore, the ENT specialist refers help-seekers to the Audiological Centre in case of a need for additional counselling, problems in accepting hearing impairment, limited willingness to use hearing aids, severe hearing loss, poor speech discrimination, and the presence of additional sensory or mental disability. The hearing aid dispenser is responsible for hearing aid fitting and is the only hearing care professionals who sells hearing aids.

\section{Costs and quality of life input}

The following costs were included in the analysis: costs of consultations with the GP assistant, GP, ENT specialist, hearing aid dispenser, and employees of the Audiological Centre (clinical physicist/audiologist, audiology assistant, social worker, and speech therapist); costs of hearing aids and hearing aid batteries, maintenance, and repair; costs of the invitation letter for telephone and internet screening; telephone costs; web hosting costs for the internet test; costs of the disposable ear cups for the HearCheck Navigator; and annual depreciation and maintenance costs of the HearCheck Navigator and audiometer used for screening. Price indices were used to convert costs to a 2011 price level (Statistics Netherlands, 2011).

Health-related quality of life was expressed as a utility score between 0 (death) and 1 (perfect health). Data on utility scores in relation to hearing thresholds and hearing aid fitting, measured with the Health Utility Index Mark 3 questionnaire, was collected by our research group in 2004 (Grutters et al., 2007). We reanalysed this data to obtain age-dependent utility scores for each of the health states. Details of this analysis can be found in the appendix.

\section{Analyses}

We examined the expected costs and effects of current practice and the 76 screening strategies for adults without hearing aids using cohort simulation. Future costs were discounted at an annual rate of $4.0 \%$ and future effects were discounted with an annual rate of $1.5 \%$ following the Dutch guidelines (Hakkaart-van Roijen et al., 2010). To account for uncertainty in the model parameter estimates, we performed probabilistic sensitivity analysis using Monte Carlo simulation with 10000 iterations. For each iteration, parameter values were drawn at random from the distribution around the base case estimates. See Appendix Table A1 and A2 for the assigned distributions.

We sorted the strategies from lowest to highest expected costs (based on the results of the probabilistic sensitivity analysis) and calculated the incremental costs and incremental QALYs of each strategy compared to current practice (no screening). Incremental cost-effectiveness ratios (ICERs) were calculated by dividing the incremental costs by the incremental QALYs. The sequence of strategies, from lowest costs to 
highest, that gives the lowest ICER values forms the cost-effectiveness frontier (Drummond et al., 2005). In a cost-effectiveness plane with the incremental costs of all strategies plotted against their incremental QALYs, the cost-effectiveness frontier is the line that connects the strategies that dominate or extendedly dominate the other strategies. A strategy is dominated by another strategy if it is more costly and less effective. A strategy is extendedly dominated if it is more costly, or less effective and has a higher ICER than a more effective strategy. The optimal strategy is the strategy on the cost-effectiveness frontier that has the highest ICER below an externally set cost-effectiveness threshold or ceiling ratio $(\lambda)$. In the Netherlands, $\lambda$ for screening is set at $€ 20000$ per QALY, which means that a screening strategy will be considered for nationwide implementation only if it costs less than $€ 20000$ per QALY gained (Van den Berg et al., 2008).

To graphically illustrate the uncertainty concerning the cost-effectiveness of the strategies, a cost-effectiveness acceptability frontier (CEAF) was constructed based on the results of the probabilistic sensitivity analysis (Fenwick et al., 2001). For construction of the CEAF, the net monetary benefit of the strategies was calculated for a range of $\lambda$ values using the following formula: net monetary benefit $=\lambda * Q A L Y-$ costs. For every value of $\lambda$, the optimal strategy was identified (that is, the strategy with the highest mean net monetary benefit for each of the 10000 iterations). Next, the probability for the optimal strategy to be the most cost-effective strategy at a certain value of $\lambda$ was calculated as the proportion of iterations in which the optimal strategy had the highest net monetary benefit. The CEAF shows the optimal strategies and their probability of cost-effectiveness for $\lambda$ values between $€ 0$ and $€ 20000$ per QALY.

\section{One-way sensitivity analyses}

In the base case analysis, the probability of screen participation was 0.33 for telephone screening and 0.44 for internet screening (both estimates were derived from a study by Koopman and colleagues (2008)). We performed a one-way sensitivity analysis to determine whether the results would have been different if the probability of screen participation for internet screening had been 0.33 as well (one-way sensitivity analysis 1). For screen-positive adults with moderate to severe hearing impairment, the probability of help-seeking after telephone and internet screening was 0.81 and 0.79 , respectively, according to Koopman and colleagues (2008). Smits and colleagues (2006) reported a probability of 0.57 . A second sensitivity analysis was performed to determine whether the results would have been different if Smits' instead of Koopman's estimates had been used (one-way sensitivity analysis 2 ). In the base case analysis we assumed that the probability that someone who sought help after telephone or internet screening continues help-seeking would be $25 \%$ higher (relative increase) than the probability of continued help-seeking in current practice. An increase is to be expected, but the size of this increase was an arbitrary choice. With a third sensitivity analysis we examined whether the results would have been different if the relative increase had been set at $10 \%$ instead of $25 \%$ (one-way sensitivity analysis 3). A rescreen after five years is less effective in stimulating help-seeking than the first 
screen (Van den Berg et al., 1999). In the base case analysis, we assumed that the effect of a rescreen after a 10-year interval would be similar to the effect of a rescreen after a 5-year interval and thus be lower than the effect of the first screen. However, given the large time interval, this assumption might not necessarily be true. We therefore reran the model with the effect of a rescreen after a 10-year interval being adapted to equal the effect of the first screen (one-way sensitivity analysis 4). Furthermore, we examined whether the results would have been different if the future costs and effects had not been discounted (one-way sensitivity analysis 5 ). In addition, we performed a threshold analysis to determine the maximum costs per screen at which the telephone and internet screening strategies would still be cost-effective compared to no screening.

\section{Results}

\section{Base case results}

Table 1 presents the results of the probabilistic sensitivity analysis. The incremental costs of the screening strategies compared to no screening ranged from $€ 4$ to $€ 59$ and the incremental QALYs ranged from 0.0003 to 0.0104 . The ICERs of all the screening strategies compared to current practice were below $€ 20000$ per QALY, indicating that screening is cost-effective as compared to no screening. The HearCheck and audiometric screening strategies were dominated by the telephone and internet screening strategies. The telephone screening strategies were either dominated or extendedly dominated by the internet screening strategies. Only internet screening strategies were on the cost-effectiveness frontier (Figure 2). With an ICER of $€ 3699$ per QALY, internet screening at age 50, repeated at ages 55,60,65, and 70 was the most cost-effective strategy at a ceiling ratio of $€ 20000$ per QALY.

Figure 3 shows the CEAF. At $\lambda$ values of $€ 3000$ per QALY or lower, current practice was the optimal strategy. At $\lambda$ values between $€ 3000$ and $€ 4500$ per QALY, the strategies that result in the highest mean net monetary benefit had a probability of being the most cost-effective between $7 \%$ and $12 \%$. At $\lambda$ values between $€ 4500$ and $€ 20000$ per QALY, internet screening at age 50, repeated at ages 55,60,65, and 70 was the optimal strategy. At a threshold of $€ 20000$ per QALY this strategy was with $100 \%$ certainty cost-effective compared to current practice and with $69 \%$ certainty the most cost-effective among all strategies. 


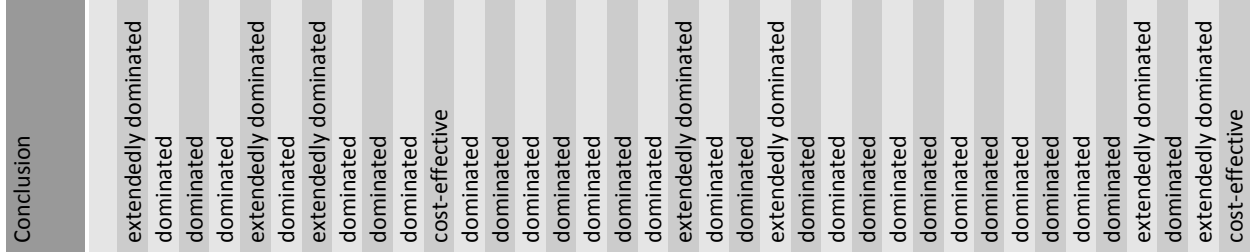

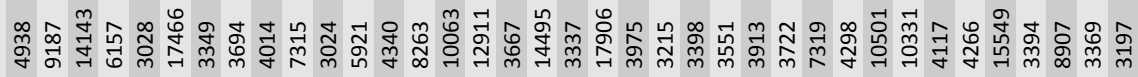
边边 f

సิ

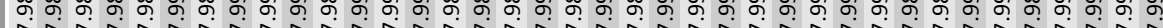

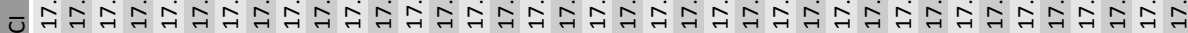

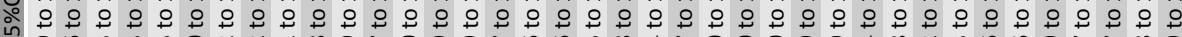
ผ

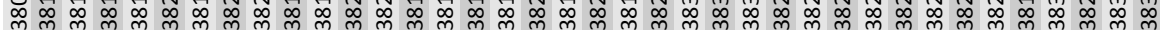

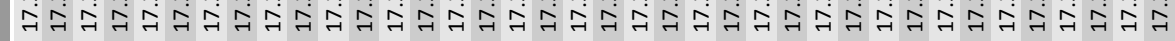

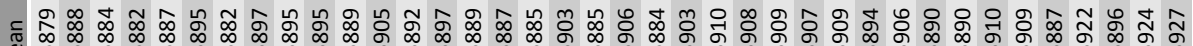

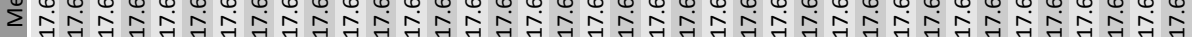

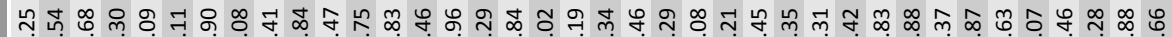
ত

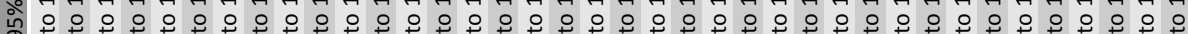

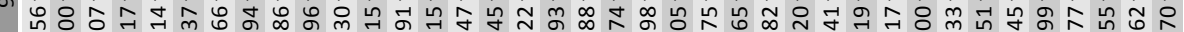

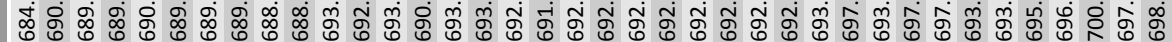

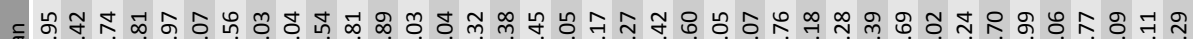

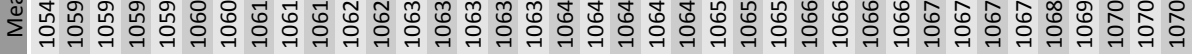

도은

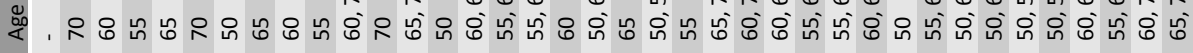

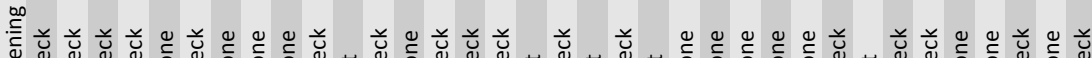

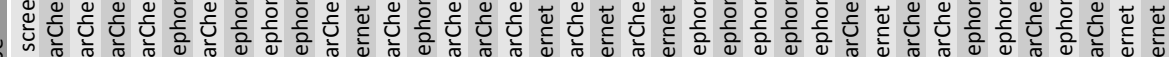

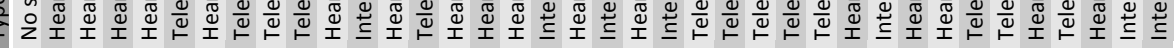




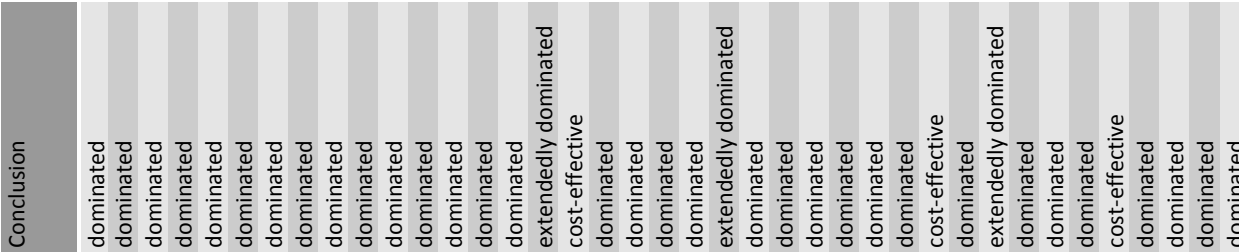

穴

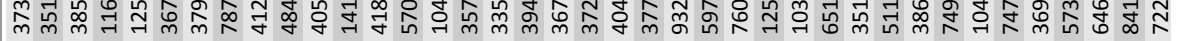

\section{ไทழ ษ

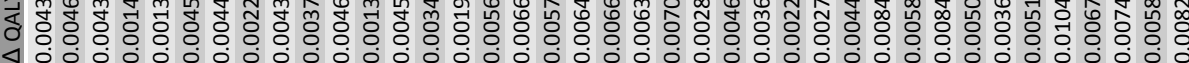 ஜ 그 m

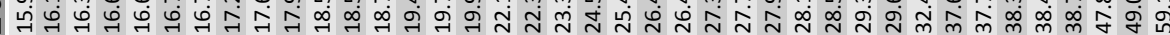

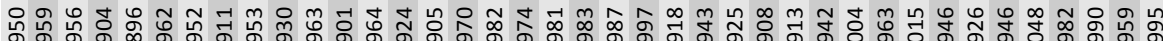

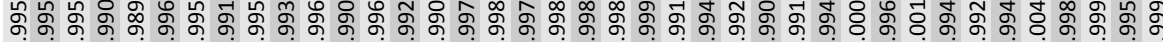
व

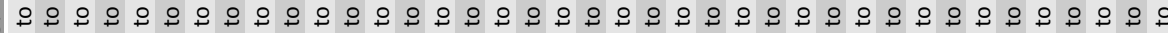
๙ু

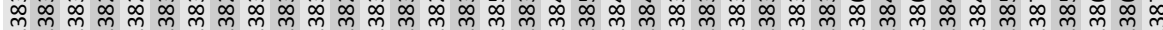
નં

สีปีส

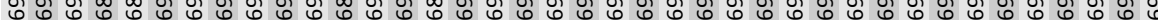

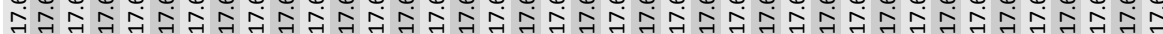

유소 ఐ ஸिं 等

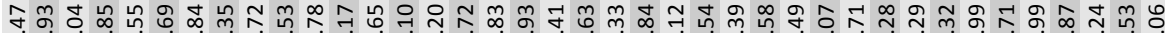

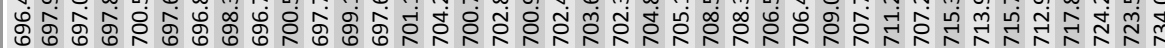

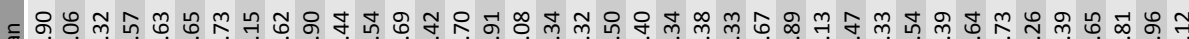

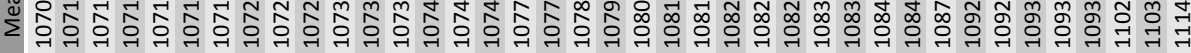

웅 ทู่ำ นู ทู่ ชั

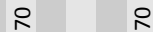
는 ㄴำ 은 ทึ่ 8ㄴ
ᄂ

రิ่น

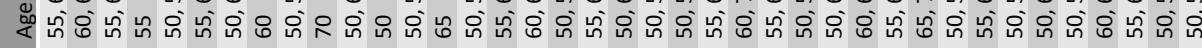

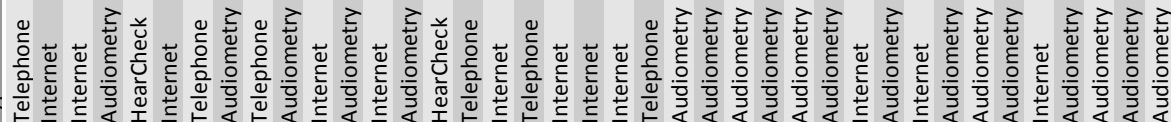




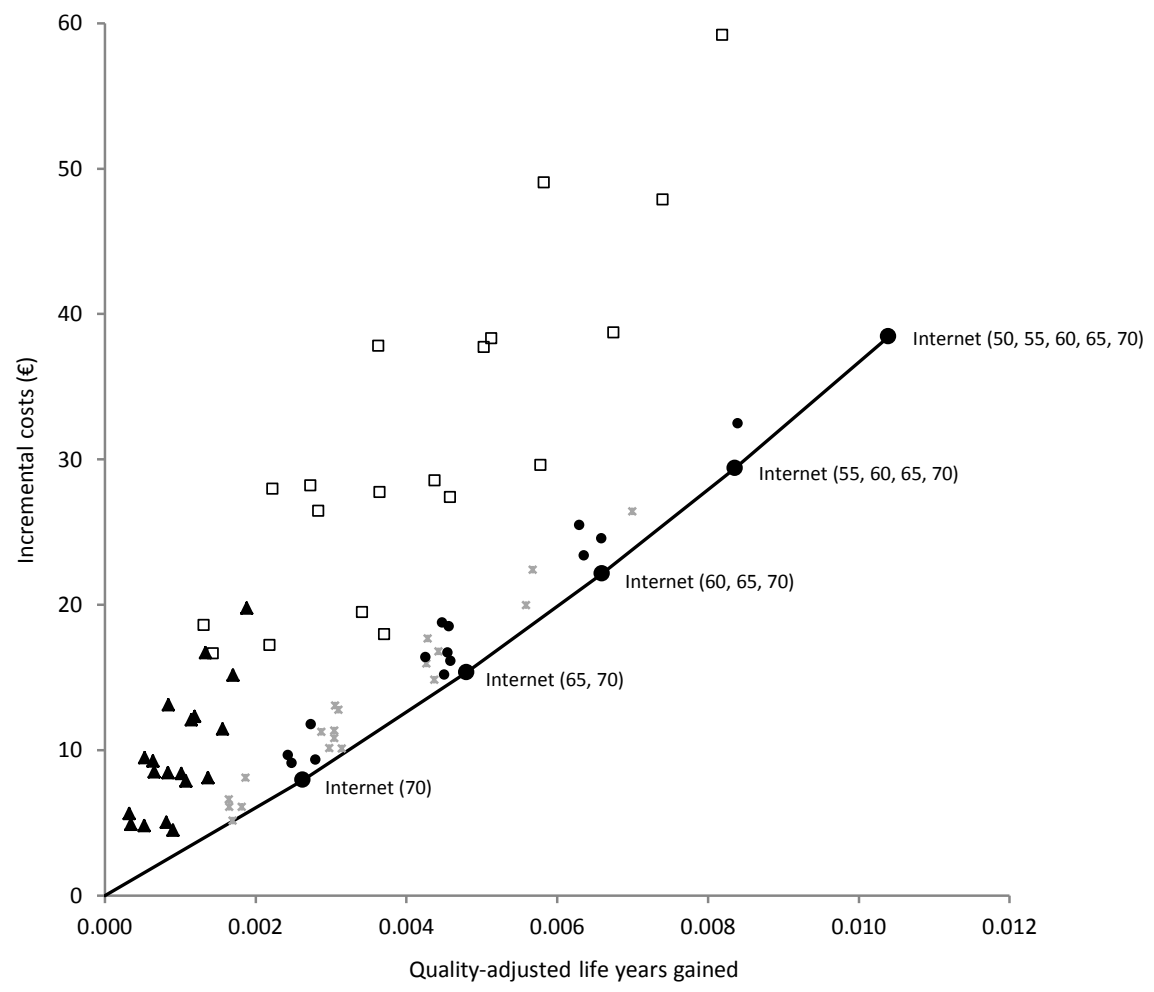

Figure 2. Cost-effectiveness plane

The incremental costs and QALYs of telephone screening (stars), internet screening (circles), HearCheck screening (triangles), and audiometric screening (squares) compared to no screening are presented. The line represents the cost-effectiveness frontier. The figure is based on the results of the probabilistic sensitivity analysis.

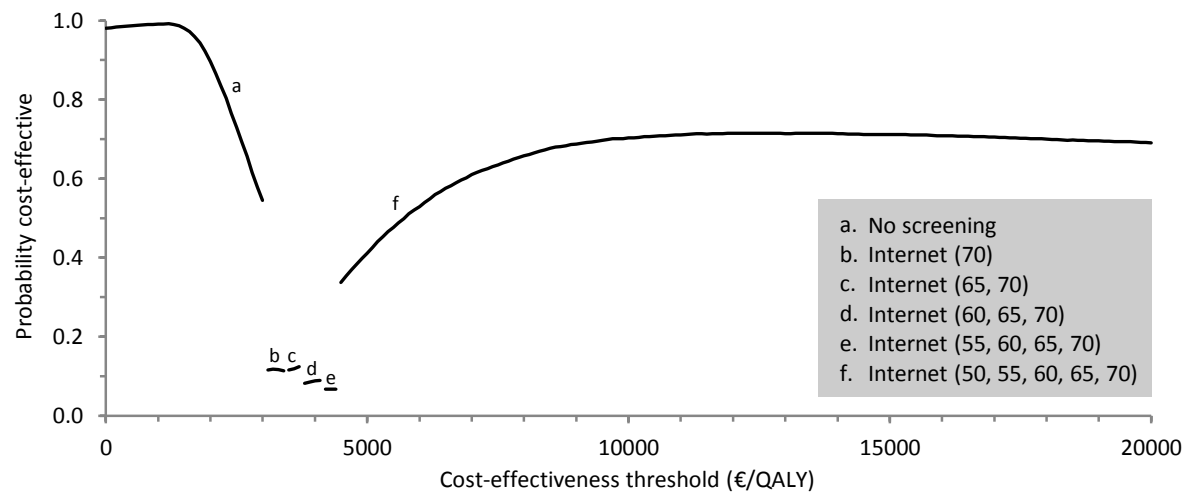

Figure 3. Cost-effectiveness acceptability frontier 


\section{One-way sensitivity analyses}

Lowering the probability of screen participation for internet screening from 0.33 to 0.44 decreased the incremental costs and QALYs of internet screening as compared to no screening. The QALY gain by internet screening at age 50 with repetition at ages 55 , 60,65 , and 70 became lower than the QALY gain by the audiometric screening at age 50 with repetition at ages $55,60,65$, and 70 but the difference was only 0.0001 QALY, while the incremental costs of the audiometric screening strategy were twice as high as the incremental costs of the internet screening strategy (see Appendix Table A3). Besides, the CEAF showed that internet screening had still the highest probability to be cost-effective for all $\lambda$ values between $€ 0$ and $€ 20000$ per QALY (see Appendix Figure A2). The results of sensitivity analysis 2 to 4 were similar to the results of the base case analysis (see Appendix Table A4 to A6 and Figure A3 to A5). Without discounting future costs and effects, the cost-effectiveness frontier was still formed by internet screening strategies only but strategies starting at age 50 outperformed strategies that started later. Internet screening at age 50, repeated at ages 55, 60, 65, and 70 was still the optimal strategy (see Appendix Table A7 and Figure A6).

In the base case, the costs of the telephone screening were set at $€ 1.23$ per screen ( 3.5 minutes at $€ 0.35 /$ minute). Threshold analysis on the screen costs showed that all telephone scenarios would still be cost-effective compared to no screening when the costs would have been $€ 83.74$ per screen. At $€ 271.66$ per screen, none of the telephone screening scenarios were cost-effective compared to no screening. In the base case, the annual costs of the internet screening were set at $€ 144.47$ (webhosting). Depending on the size of the target population and the participation rate, the costs per screen were between $€ 0.0013$ (for screening offered to people aged 50) and $€ 0.0026$ (for screening offered to adults aged 70 ). Threshold analysis showed that, if the costs per screen would have been $€ 92.73$, all internet screening scenarios would still be cost-effective compared to no screening. At $€ 320.49$ per screen, none of the internet screening scenarios were cost-effective compared to no screening.

\section{Conclusions}

Adult hearing screening was cost-effective compared to current practice (no screening). The internet screening strategies were the most cost-effective, closely followed by the telephone screening strategies. Internet screening probably outperformed telephone screening because of its lower test costs and higher test sensitivity and screen participation rate. Internet and telephone screening probably outperformed HearCheck and audiometric screening because of their lower test costs and accessibility for people who do not annually visit the GP. Internet access hardly forms a barrier for internet screening nowadays in the Netherlands as $98 \%$ of adults aged between 45 and 55 years, $94 \%$ of adults aged between 55 and 65 years, and $85 \%$ of adults aged between 65 and 75 years have access to internet at their home (Statistics Netherlands, 2013). 
Morris and colleagues (2013) reported an ICER of $f 1461$ ( $€$ 1680) per QALY for an audiometric screening at age 60, repeated at ages 65 and 70, compared to current practice in the United Kingdom. In our study, the ICER of this strategy was $€ 5736$ per QALY (dominated by internet screening at age 50,55, 60, 65, and 70). The difference in the ICER might be caused by differences in model input estimates. For example, the costs of a hearing aid trail and a hearing aid are much higher in the Netherlands than in the United Kingdom, and the probability of hearing aid uptake might be lower because Dutch patients have to pay a substantial amount of money for a hearing aid (on average $€ 600$ in 2011), while most patients in the United Kingdom receive hearing aids for free. Differences in model structure probably contribute to the discrepancy between the ICERs as well. We modelled screening programmes for the detection of a best-ear pure-tone average hearing loss (at $0.5,1,2$, and $4 \mathrm{kHz}$ ) of $25 \mathrm{~dB}$ or more, while Morris' screening programme aimed to detect a hearing loss of $30 \mathrm{~dB}$ or more. And unlike Morris and colleagues (2013), we included the costs of care given to people who consult a hearing care professional or even start a hearing aid trial but do not take up hearing aids. Our study showed that in a more expensive hearing care system audiometric screening is cost-effective compared to no screening. However, internet screening is the most cost-effective approach.

Liu and colleagues (2011) reported that adult hearing screening using a hand-held screening device is cost-effective. They based their conclusion on a randomised controlled trial that showed that screening led to a significant increase in hearing aid use 1 year later (2.8\%). The incremental costs of screening per additional hearing aid user were US $\$ 1439$ ( $€$ 1040). However, it cannot be concluded from this trial whether screening is indeed cost-effective since it is unknown how much society is willing to pay for one additional hearing aid user. So far, it has only been assessed how much society is willing to pay for QALYs gained. Other limitations of this trial are the short follow-up period and the fact that it included particularly male veterans, a study sample that may not be representative for the total adult population. By using a Markov model we were able to calculate the lifetime costs and effects of screening for a general population cohort. And because we measured the effectiveness in terms of QALYs gained, we could draw conclusions with regard to the cost-effectiveness of a large number of screening strategies.

Our study has several limitations. Bias may be introduced by the fact that the model parameter estimates were derived from several sources, none of which was a randomised controlled trial. However, the probabilities of screen participation for telephone screening, internet screening, and screening at the GP, and some of the probabilities of help-seeking after screening were all derived from one study (Koopman et al., 2008). The assumptions we had to make about some of the parameter estimates due to a lack of evidence may have biased the results as well. However, with probabilistic sensitivity analysis we accounted for the uncertainty in the estimates by assigning wide distributions to the model parameters and one-way sensitivity analyses showed that the results were insensitive to the assumptions we were most uncertain about. A second limitation is that the costs of management, quality control, and monitoring of the screening strategies were not taken into 
account. Since these costs will be similar for all screening strategies, inclusion of these costs would not have changed the strategy ranking. However, inclusion of these costs would have resulted in higher ICERs of the screening strategies compared to no screening. Post-hoc threshold analysis on strategy costs revealed that the strategy with internet screening at age 50,55,60,65, and 70 would still be cost-effective if the incremental costs of the strategy (compared to no screening) would increase from $€ 38$ to $€ 208$. Based on this, it seems plausible that also when the costs of management, quality control and monitoring are taken into account, the screening strategy will be cost-effective. A third limitation of the study is that the model disregarded rehabilitation options other than conventional hearing aids, like communication training or assistive listening devices. In most previous studies on adult hearing screening, rehabilitation was limited to hearing aid fitting (Pronk et al., 2011). Consequently, data on the effect of screening on uptake of other rehabilitation options was insufficient to incorporate in the model, as was data on quality of life gain by other rehabilitation options. Future research to address this topic is recommended.

The utility estimates used in the model were based on the Health Utility Index Mark 3 questionnaire. This is the recommended instrument for measuring utility scores to assess health-related quality of life in hearing impairment because of its good validity and responsiveness (Yang et al., 2013). Other multi-attribute utility instruments, like the HUI2, EQ-5D, and SF-6D, are less sensitive to changes in utility scores after hearing aid fitting or even unable to measure a change at all (Barton et al., 2004; Grutters et al., 2007; Joore et al., 2003b). If another multi-attribute utility instrument had been used, adult hearing screening may not have been cost-effective. Although we used the most sensitive utility instrument, the screening strategies led to only little gain in quality of life (0.0003 to 0.0104 QALYs). QALY gains from population screening programmes generally tend to be quite modest (Kim et al., 2007; Maeda et al., 2004), probably because the effect of treatment is diluted by the large number of healthy people in the target population for whom no quality of life gain can be achieved.

To what extent our results can be generalized and transferred to other countries depends on international similarities and differences with regard to health care system and culture. For example, hearing aid uptake after screening may be higher in countries were hearing aids are provided free-of-charge, like in the United Kingdom or Denmark. And in countries were patients' financial contribution to hearing aids is higher than in the Netherlands, for example in Belgium, screening will probably be less cost-effective because the high hearing aid costs may withhold screen participants from taking up hearing aids. Also the likelihood to participate in hearing screening differs between countries. Koopman and colleagues (2008) found that, compared to Dutch adults, British and German adults were much more likely to do a telephone test ( $50 \%$ and $76 \%$ versus $33 \%$ ) or internet test (65\% and $73 \%$ versus $44 \%$ ). And while Dutch and British adults were found to be equally likely to opt for additional hearing testing after a positive screen (approximately 80\%), the Germans were much more likely to do so (approximately 95\%) (Koopman et al., 2008). Furthermore, the effectiveness of internet screening will depend on the proportion adults in the target population who have internet access at home. The Netherlands are leading in Europe 
with $95 \%$ of the households having internet access, followed by Luxembourg (94\%), Denmark, and Sweden (both 93\%). Internet access in Europe is lowest in Bulgaria (54\%), Greece (56\%), and Romania (58\%) (Eurostat, 2013).

In conclusion, this study suggests that nationwide adult hearing screening programmes are (in comparison with no screening) cost-effective interventions to increase the quality of life of adults with hearing loss, with internet screening at age 50 , repeated at ages $55,60,65$, and 70 being the optimal strategy. Since at a threshold of $€ 20000$ per QALY this strategy was with $100 \%$ certainty cost-effective compared to current practice and with $69 \%$ certainty the most cost-effective among all strategies, policymakers might consider nationwide implementation. Telephone screening is a good alternative for people without internet access. 


\section{Appendix}

This appendix gives an elaborate description of our considerations, calculations, and assumptions with regard to the model parameter estimates. Table A1 lists the model parameters used to calculate the cost and effects of hearing care in current practice, including the base case parameter estimates, distributions, and references to data sources. Table A2 lists the additional model input for the screening specific parameters. Table A3 to A7 and Figure A2 to A6 present the results of the one-way sensitivity analyses. References are listed at the end of this document.

\section{Hearing acuity}

We retrieved population-based data on the prevalence and incidence of audiometrically assessed mild and moderate to severe hearing impairment in men and women from the Australian Blue Mountains Hearing Study to calculate the overall prevalence and 1-year incidence, taking into account the gender distribution in the Dutch population (Mitchell et al., 2011; Statistics Netherlands, 2011). We combined these prevalence estimates with information on hearing aid ownership, collected by our research group in 1998 (Duijvestijn et al., 2003), to obtain prevalence estimates of hearing impairment for adults without hearing aids specifically. The incidence of hearing impairment for people aged between 50 and 54 years was assumed to be half as high as for people aged between 55 and 59 years.

\section{Current practice}

In the Netherlands, $64 \%$ of the adults who seek help for their hearing complaints initially turn to the GP while the others initially visit the hearing aid dispenser (Groenland, 2011). The next step in the help-seeking process depends on the preference of the help-seeker and the professional judgement of the GP or hearing aid dispenser with regard to the need for involvement of the ENT specialist. Some people do not continue help-seeking, others start a hearing aid trial immediately or consult the ENT specialist (Figure A1). The ENT specialist clinically examines the ear, removes ear wax, diagnoses ear pathology, performs hearing tests (pure-tone audiometry), informs the help-seeker about rehabilitation options, and prescribes hearing aids to those who are willing to try them. Furthermore, the ENT specialist refers help-seekers to the Audiological Centre in case of a need for additional counselling, problems in accepting hearing impairment, limited willingness to use hearing aids, severe hearing loss (pure-tone average at 1,2 , and $4 \mathrm{kHz}$ of $70 \mathrm{~dB}$ or more in the best ear), poor speech discrimination (less than $70 \%$ for monosyllables), and the presence of additional sensory or mental disability. These referral criteria are set out in the Dutch guidelines for hearing care professionals (Nationaal Overleg Audiologische Hulpmiddelen, 2009). 


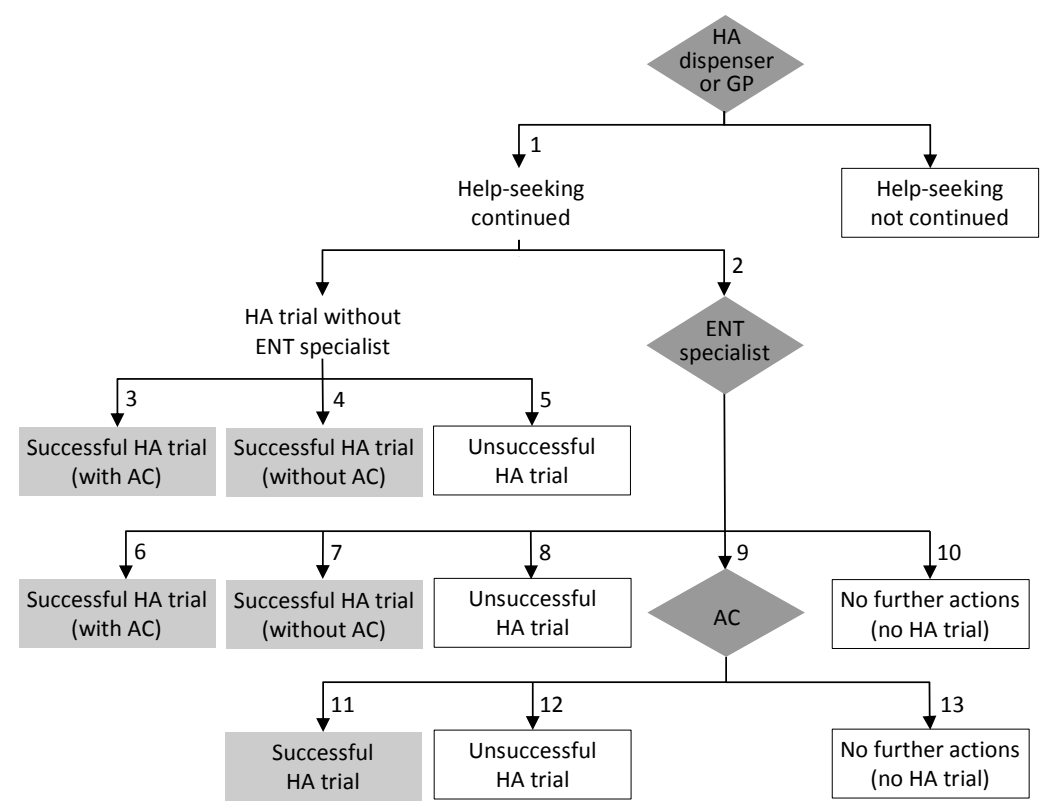

Figure A1. Possible paths in continued help-seeking

The dark grey rhombi represent contacts with health care professionals and the light grey rectangles represent hearing aid uptake. AC, Audiological Centre; ENT, Ear Nose Throat; GP, general practitioner; $\mathrm{HA}$, hearing aid.

At the Audiological Centre, clinical physicists with a post academic degree in audiological and psychosocial rehabilitation cooperate with speech therapists, social workers, and psychologists to provide help-seekers with the best possible care for their hearing and hearing-related problems. During the first visit to the Audiological Centre, all helpseekers have a consultation with the clinical physicist and a consultation with the audiology assistant for pure-tone audiometry, speech audiometry, hearing aid advice (if interested in starting a hearing aid trial), and hearing aid adjustment (if a hearing aid trial was started already). Of all the adults who visit the Audiological Centre, approximately $2 \%$ consult the speech therapist for hearing training and $2 \%$ consult the social worker (expert opinion).

The hearing aid dispenser is responsible for hearing aid fitting and is the only hearing care professional who sells hearing aids. People who start a hearing aid trial after having visited the Audiological Centre always have a follow-up visit at the Audiological Centre at the end of the trial period. People who start a trial on the advice of the ENT specialist have a follow-up visit at the ENT specialist if the trial resulted in hearing aid uptake (successful trial). In case of an unsuccessful trial, thus a trial that did not result in hearing aid uptake, a follow-up visit at the ENT specialist is not necessary. A hearing aid trial lasts on average 6 to 8 weeks. For trials that last longer than 3 months, the hearing aid dispenser refers the patient to the Audiological Centre. Help-seekers who visit the Audiological Centre, either before having started a hearing aid trial or for 
assistance during a difficult trial, have on average 1.22 follow-up visits (Duijvestijn et al., 2011). These follow-up visits include a consultation with the audiology assistant for hearing aid adjustment and a consultation with the clinical physicist.

For the model, we used data on the probability to seek help and the probability to continue help-seeking collected by our research group in 1998 (Duijvestijn et al., 2003). Data on probabilities related to the outcome of the help-seeking process, the pathway followed (arrow 2 through 13 in Figure A1), and the hearing care professionals involved were collected by our research group in 2002 (Duijvestijn et al., 2011). We reanalysed these two datasets to obtain estimates of probabilities related to (continued) help-seeking, hearing aid purchase and resource use in current practice for each of the health states separately. The probability for hearing aid replacement in the fifth, sixth, and seventh year after purchase were retrieved from Dreschler and colleagues (2009). We assumed that everyone who had not replaced a hearing aid in the fifth, sixth, or seventh year after purchase would do so in the eighth year. Furthermore, we assumed that people who seek help for hearing aid replacement would not drop out of the help-seeking process and would all end up with new hearing aids. The hearing aid discard probability is 6\% (Van den Brink et al., 1996).

\section{Screening strategies}

We derived probability estimates for screen participation from a study on intentional behaviour (Koopman et al., 2008). For the HearCheck and audiometric screening strategies at the GP practice, the annual probability to consult the GP was included in the model to account for the fact that not all members of the target population visit the GP annually (Statistics Netherlands, 2011). Information on the validity of the telephone and internet version of the National Hearing Test was retrieved from the papers by Smits and colleagues (2004) and Leensen and colleagues (2011), respectively. To obtain data on the validity of the Siemens HearCheck Navigator, we screened the hearing of 166 adults (332 ears) who visited the Audiological Centre of the Maastricht University Medical Centre for audiometry in February and March 2013.

Only three Dutch studies have addressed help-seeking behaviour after adult hearing screening (Koopman et al., 2008; Smits et al., 2006; Van den Berg et al., 1999). Smits and colleagues (2006) studied help-seeking after telephone screening. The telephone test informed people that their hearing was either poor, insufficient, or good. Fourteen percent of the people who scored good, $46 \%$ of the people who scored insufficient, and $57 \%$ of the people who scored poor sought help. Koopman and colleagues (2008) also studied help-seeking after screening and found that respectively $81 \%$ and $79 \%$ of the people who screen positive on the telephone and internet test would seek help. In the base case analysis, we used Koopman's estimates for the probability that people with a moderate to severe hearing impairment seek help when screened positive on the telephone or internet test because these estimates seem more realistic than Smits' estimates compared to the help-seeking probability in current practice. We performed a sensitivity analysis to determine whether the results would have been different if Smits' instead of Koopman's estimates had been used (one-way sensitivity analysis 2). 
For the probability that people with normal hearing or with a unilateral or mild hearing impairment seek help when screened positive on the telephone or internet test, we used the estimate of Smits and colleagues (2006) for people who scored insufficient, assuming that the people with a moderate to severe hearing impairment would have scored poor and that help-seeking after internet screening would be comparable to help-seeking after telephone screening.

The studies of Smits and colleagues (2006) and Koopman and colleagues (2008) did not report on the probability that people who sought help after screening continue helpseeking by starting a hearing aid trial or consulting an ENT specialist. We assumed a relative increase of $25 \%$ in the probability that a help-seeker continues help-seeking for the telephone and internet screening strategies compared to current practice because screen participants have objective proof of hearing impairment and Dutch GPs are more likely to refer someone with objectively assessed hearing impairment to a hearing care professional than someone with subjective hearing complaints alone (Eekhof et al., 2000). An increase is to be expected, but the size of this increase was an arbitrary choice. With a sensitivity analysis we examined whether the results would have been different if the relative increase had been set at $10 \%$ instead of $25 \%$ (oneway sensitivity analysis 3 ). For the HearCheck and audiometric screening strategies at the GP practice, the probability that the screen participants discuss their hearing with the GP (i.e. the probability of help-seeking) was set at $100 \%$ because the GP initiates the discussion. The probability that they continue help-seeking would probably be lower than in current practice because they had actually visited the GP for health complaints other than their hearing. We assumed that their probability to continue help-seeking would equal the probability that someone in current practice starts seeking help.

The third study on help-seeking after screening, a study by Van den Berg and colleagues (1999), was not used as data source for probabilities of (continued) helpseeking because the type of screening was too different from our four screening types. However, this study provided useful information on the relative effectiveness of rescreening 5 years after an initial screening. They found that after a first screen $24.3 \%$ of the screen participants had sought help and $21.5 \%$ had continued help-seeking. After a rescreen 5 years later, only $16.8 \%$ of the participants had sought help and $13.9 \%$ had continued help-seeking. Thus the probability to seek help had decreased with a factor 0.31 and the probability to continue help-seeking had decreased with a factor 0.35. For the telephone and internet screening strategies, we calculated the probability of help-seeking after a rescreen by multiplying the difference between the probability after the first screen and the probability in current practice with a factor 0.31 and subtracting this from the probability after the first screen. For the HearCheck and audiometric screening strategies, we kept the probability of help-seeking after a rescreen equal to the probability after the first screen (1.00) because the GP initiates the discussion, but we reduced the probability of continued help-seeking with a factor 0.35. We assumed that probabilities of (continued) help-seeking in repeated screening strategies with 10-year intervals would be similar to those in repeated screening strategies with 5-year intervals. However, given the large time interval, this 
assumption might not necessarily be true. We therefor reran the model with the effect of a rescreen after a 10-year interval being adapted to equal the effect of the first screen (one-way sensitivity analysis 4).

\section{Health-related quality of life}

The outcome measure in the model is the quality-adjusted life year (QALY) which is a combined measure of health-related quality of life and duration of life. Health-related quality of life is expressed as a utility score between 0 (death) and 1 (perfect health). QALYs were calculated by multiplying the time spend in each health state with the utility score attached to this health state in each cycle and summing up over all cycles. We used data on utility scores in relation to hearing thresholds and hearing aid fitting, measured with the Health Utility Index Mark 3 questionnaire, that was collected by our research group in 2004 (Grutters et al., 2007). For the Health Utility Index Mark 3 questionnaire no age-dependent norm utility values are available. To obtain age dependent utility scores for people with normal hearing (not present in the dataset), we set the score on the hearing item of the Health Utility Index on 1.00 (no hearing difficulties) for all people in the dataset $(\mathrm{N}=211)$ and recalculated the overall utility scores. Then, the original and modified dataset were merged. We reanalysed this new dataset using ordinary least square linear regression to obtain a regression formula that expresses the relationship between utility scores and age for people without hearing aids with normal hearing, unilateral hearing impairment, mild hearing impairment, and moderate to severe hearing impairment separately. The model explained $16 \%$ of the variance in the utility scores. The regression coefficients with their standard errors are given in Appendix Table A1. Cholesky decomposition was used for the distribution around the utility scores. To prevent that people with moderate to severe hearing impairment would have higher utility scores than people with mild hearing impairment in the probabilistic sensitivity analysis, the model was programmed in such a way that, if this was the case, the estimate for moderate to severe hearing impairment was set equal to the utility score estimate for people with mild hearing impairment.

\section{Covariance matrix (used for Cholesky decomposition)}

$\begin{array}{lllll} & \text { Age } & \text { DumUnilateralHI } & \text { DumMildHI } & \text { DumModSevHI } \\ \text { Age } & 1.375^{*} 10^{-6} & & & \\ \text { DumUnilateralHI } & 8.153^{*} 10^{-6} & 2.079 * 10^{-3} & & \\ \text { DumMildHI } & -8.031^{*} 10^{-7} & 1.980^{*} 10^{-4} & 6.270 * 10^{-4} & \\ \text { DumModSevHI } & -7.235^{*} 10^{-6} & 1.600^{*} 10^{-4} & 2.070^{*} 10^{-4} & 9.550^{*} 10^{-4}\end{array}$

The dataset also contained information on the change in health-related quality of life after hearing aid fitting. The average change was calculated for people with mild and moderate to severe hearing impairment separately. The number of people with unilateral hearing impairment in this dataset was too small to calculate the effect of hearing aid fitting on quality of life for this group. We assumed that the effect of hearing aid fitting on quality of life for people with unilateral hearing impairment would be equal to the effect on quality of life for people with mild hearing impairment 
since the utility scores of people without hearing aids with unilateral and mild hearing impairment were also equal. Furthermore, we assumed that the effect of hearing aid fitting on an individual's quality of life after screening would be equal to that in current practice.

\section{Costs}

The letter used to invite people to do the telephone or internet test costs $€ 0.48$ (market price). The telephone test costs $€ 0.35 /$ minute and takes on average 3.5 minutes (Smits \& Houtgast, 2005). Web hosting costs $€ 144.47$ per year (market price). We calculated the costs of the internet screening per participant by dividing the annual web hosting costs by the expected number of screen participants nationwide. Disposable ear cups for the HearCheck Navigator cost $€ 0.25$ each (market price; one ear cup needed per participant). The market price of the HearCheck Navigator is $€ 165$. We set the price of the audiometer at $€ 1908$ (market price of the Audiometrics AS608e screening audiometer). The annual costs of the HearCheck Navigator and audiometer amount to $18 \%$ of their new price: $10 \%$ depreciation and $8 \%$ maintenance (Hakkaartvan Roijen et al., 2010). The costs of the HearCheck Navigator and audiometer per participant were calculated by dividing the annual depreciation and maintenance costs by the expected number of screen participants per GP practice.

The costs of a consultation with the GP, ENT specialist, social worker, and speech therapist were based on the Dutch manual for cost research (Hakkaart-van Roijen et al., 2010). We calculated the costs of the clinical physicist, audiology assistant, and GP assistant by multiplying the mean duration of their activities by their average labour costs and added a surcharge of $42 \%$ to account for costs of standard instruments, materials, housing, and overhead, as recommended in the Dutch manual for cost research (Hakkaart-van Roijen et al., 2010).

Based on the annual report from the Dutch association of hearing aid dispensers (Nederlandse Vereniging van Audicien Bedrijven, 2011b), we estimated the hourly costs of the hearing aid dispenser to be $€ 87.50$ (including the costs of standard instruments, materials, housing, and overhead). According to this report, a hearing aid dispenser spends on average 6 to 8 hours to a hearing aid applicant and the costs of care delivered by the hearing aid dispenser and hearing aids combined amount to $€$ 1017. We assumed that the initial assessment lasts 30 minutes and that a monaural and binaural hearing aid trial (including aftercare) last 5.5 and 7.5 hours, respectively. The annual aftercare costs per hearing aid are $€ 13$ and the average hearing aid replacement time is 5.62 years (Dreschler et al., 2009). Approximately $80 \%$ of the hearing aid owners have hearing aids for both ears (Boer\&Croon, 2010; Van Thiel, 2010). We combined this information to calculate the costs of the initial assessment at the hearing aid dispenser, the trial costs, and the hearing aid costs. The annual costs of hearing aid use (including the costs of batteries, maintenance, and aftercare) and the annual probability of hearing aid repair were based on the report of Dreschler and colleagues (2009). The average costs of hearing aid repair were derived from an online database of the Health Care Insurance Board (2011). 


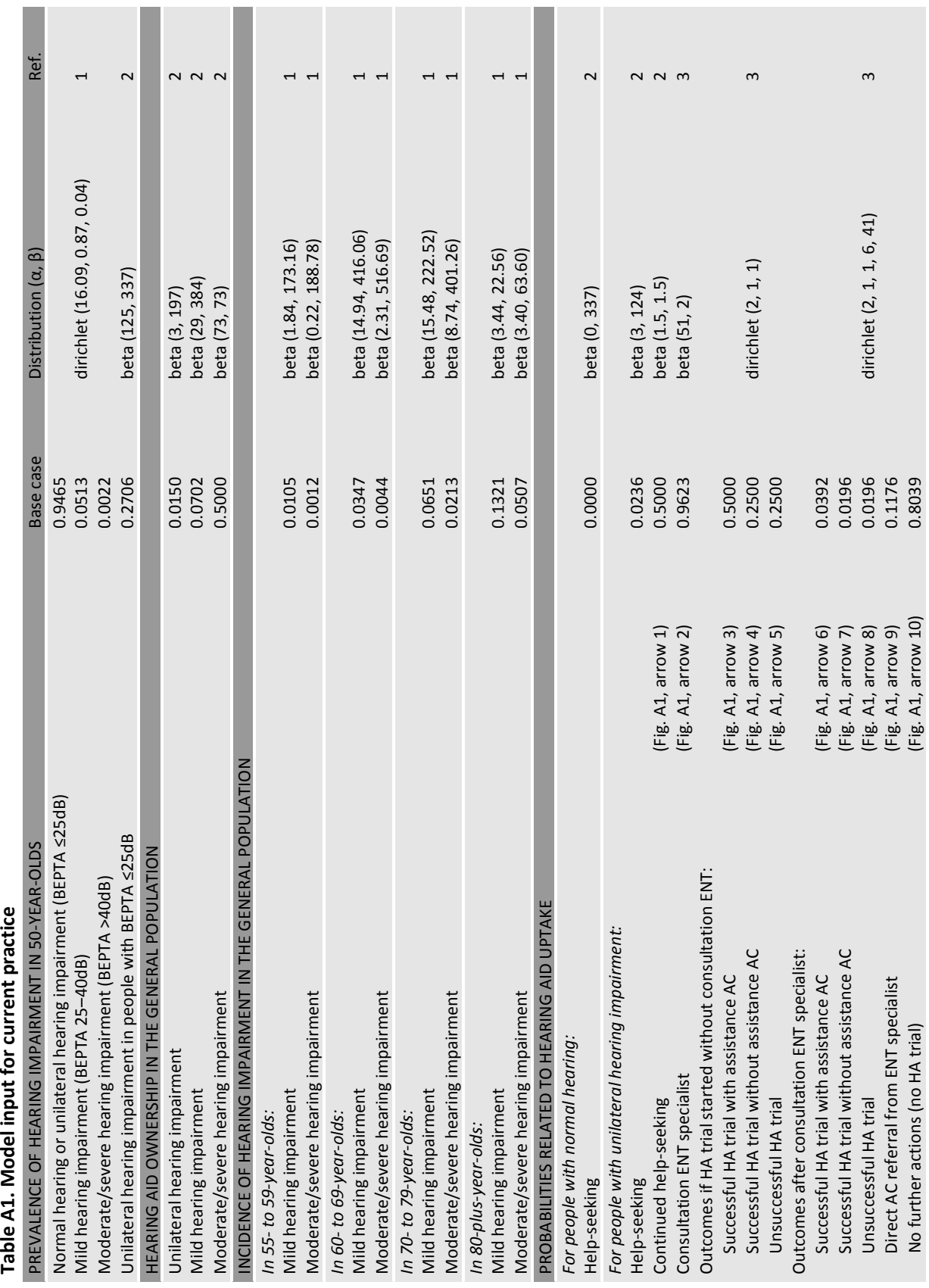




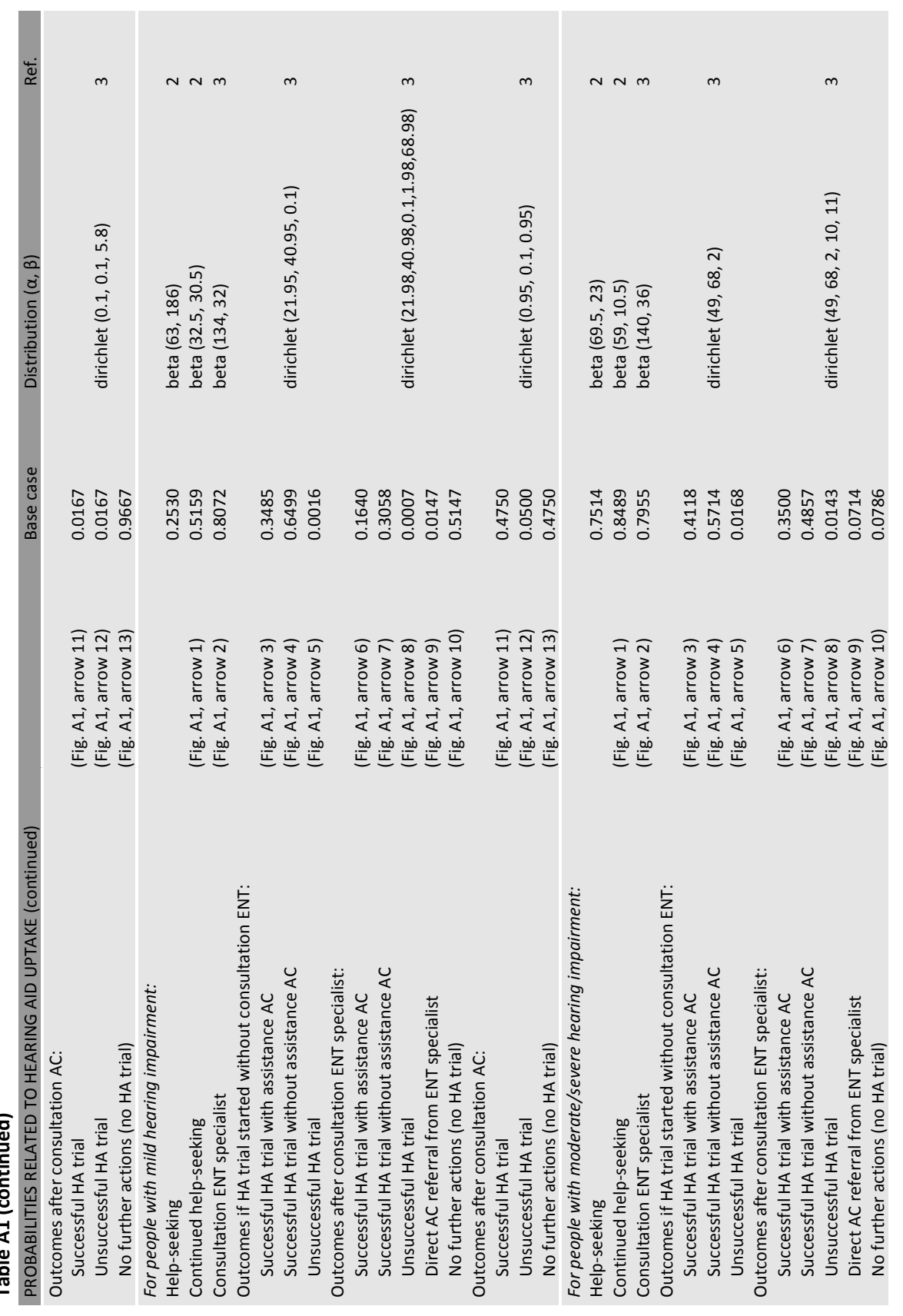




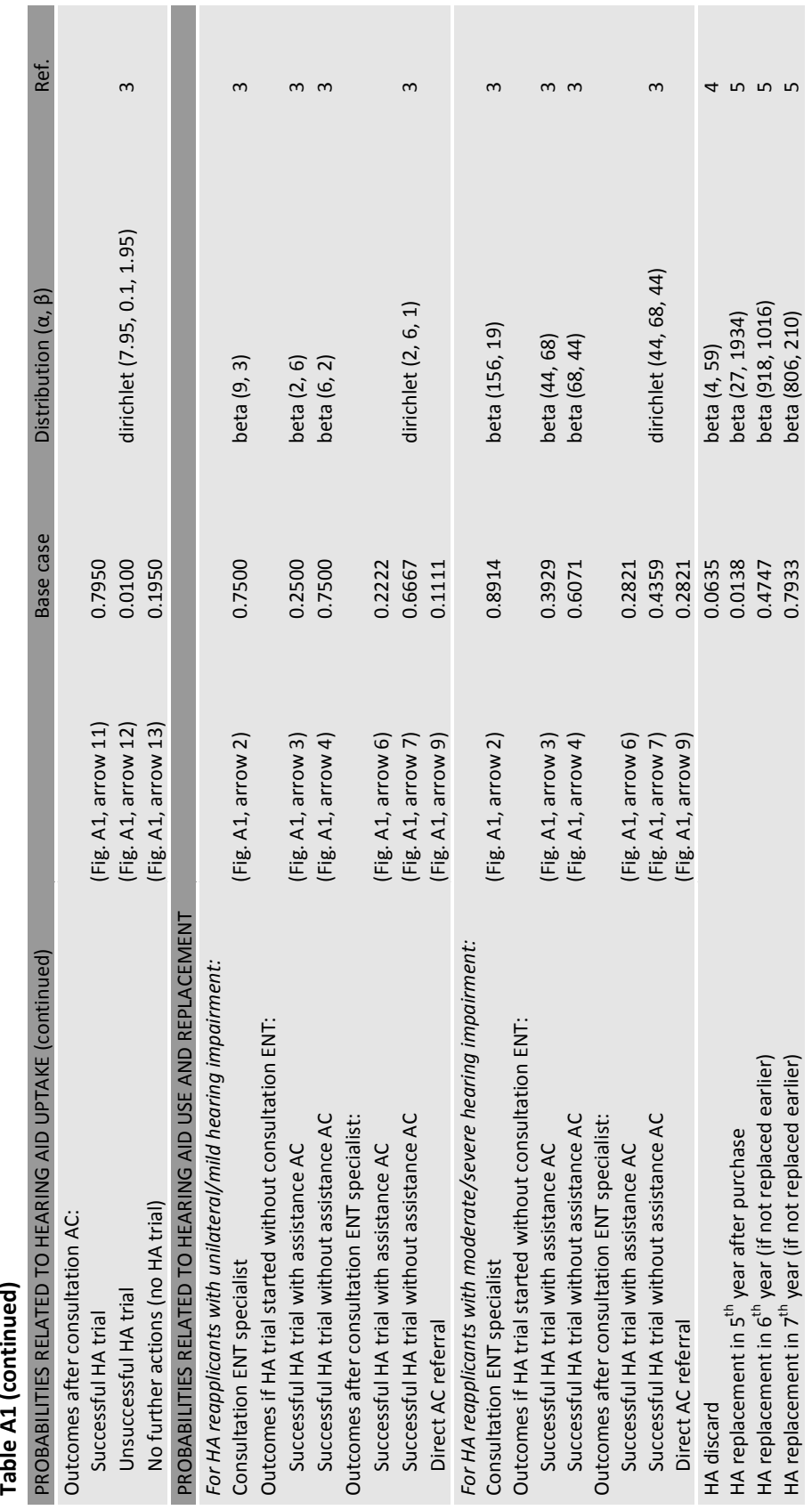




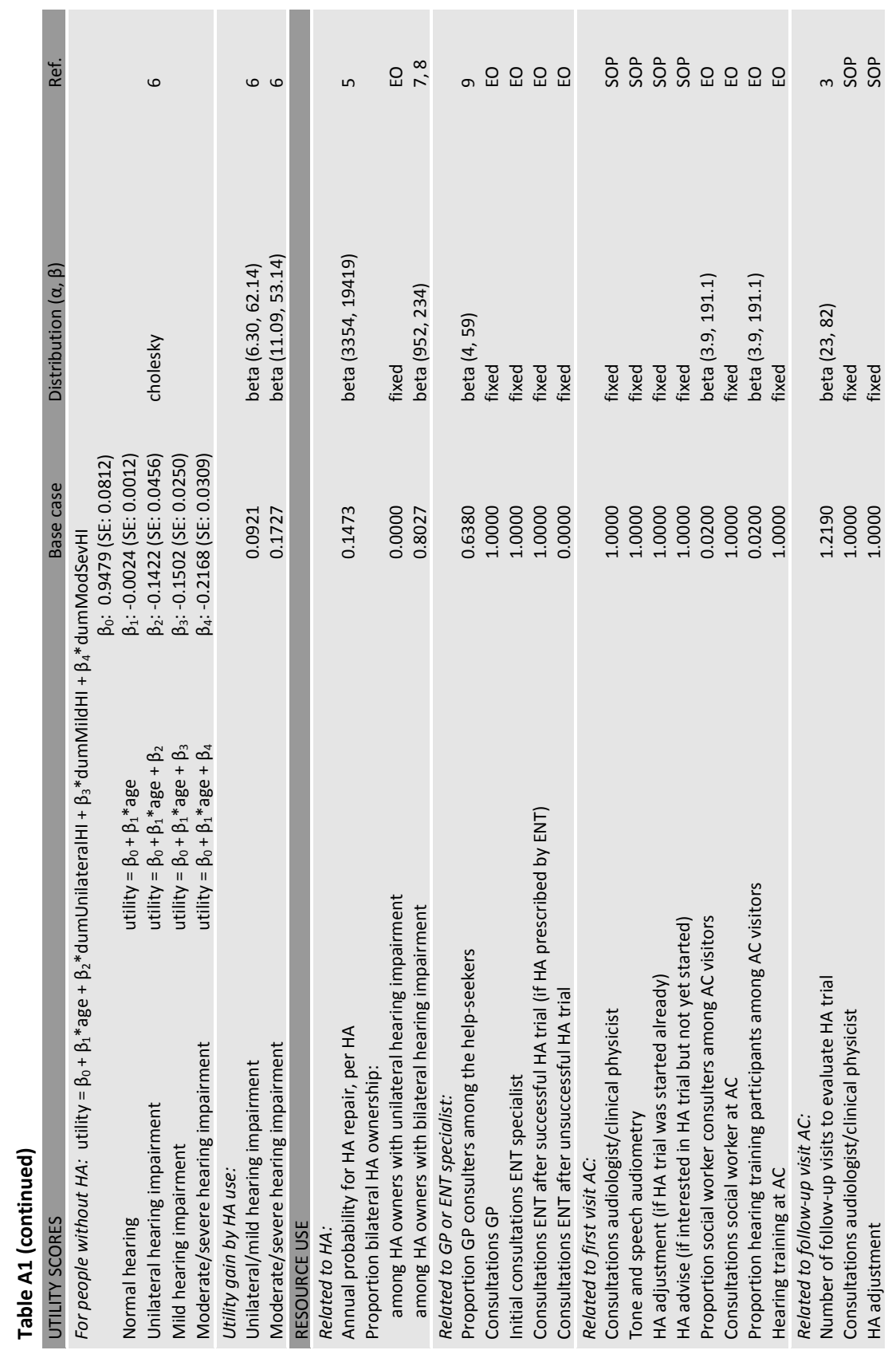




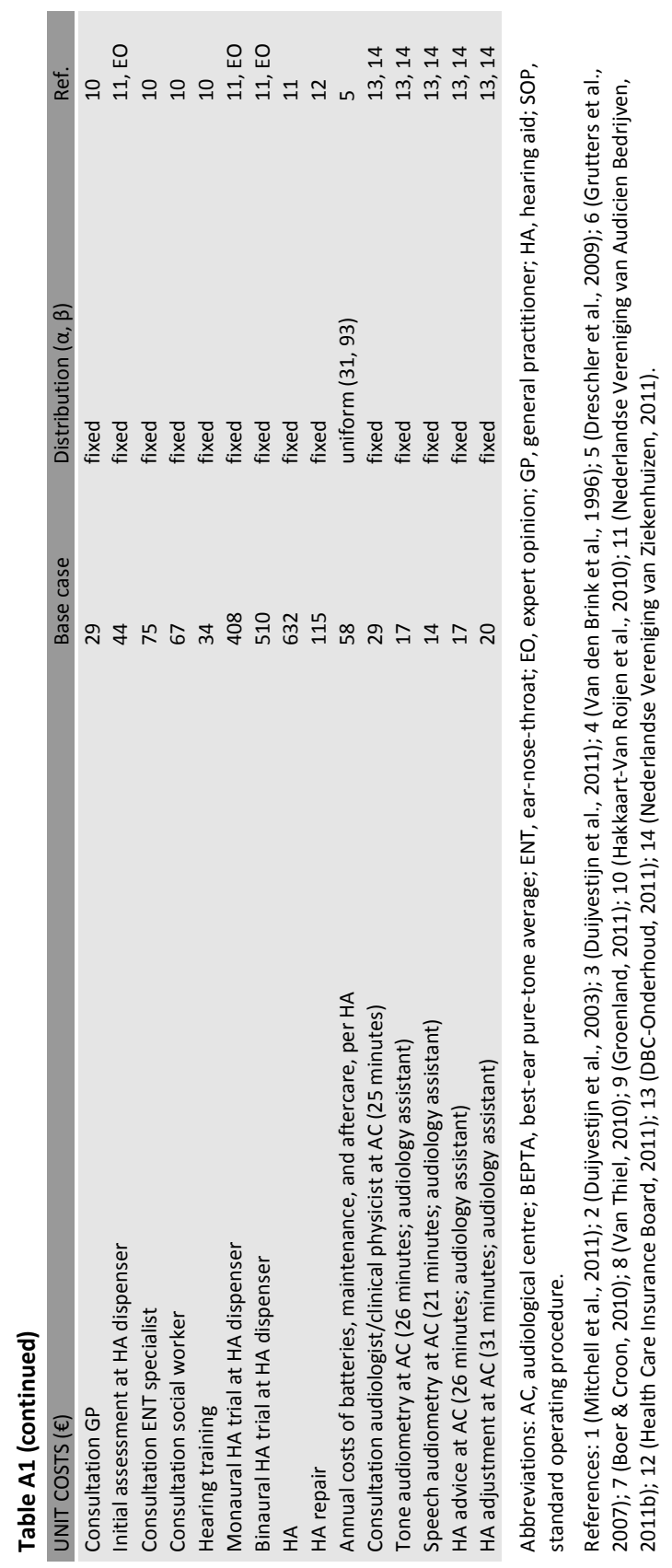




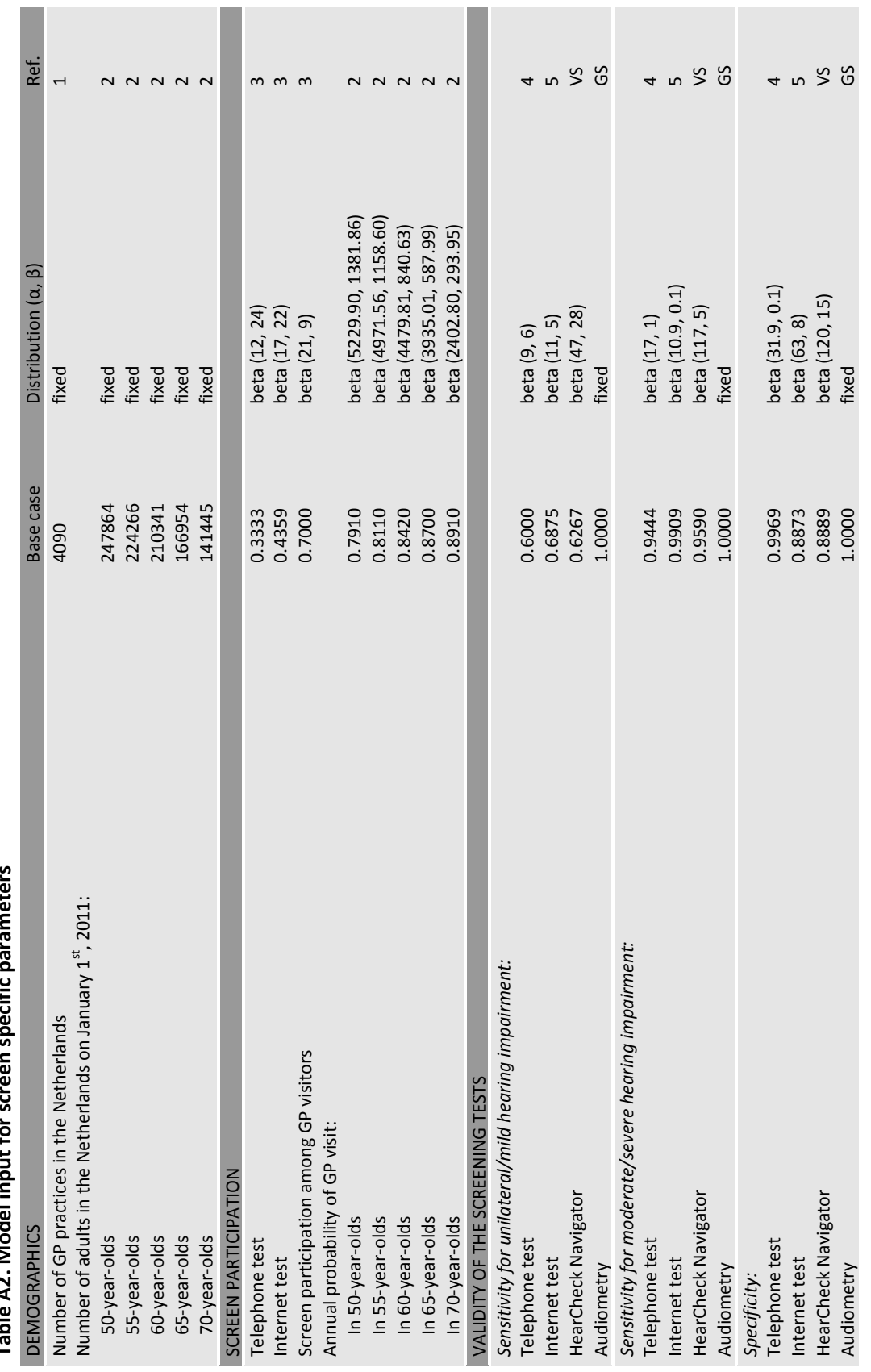




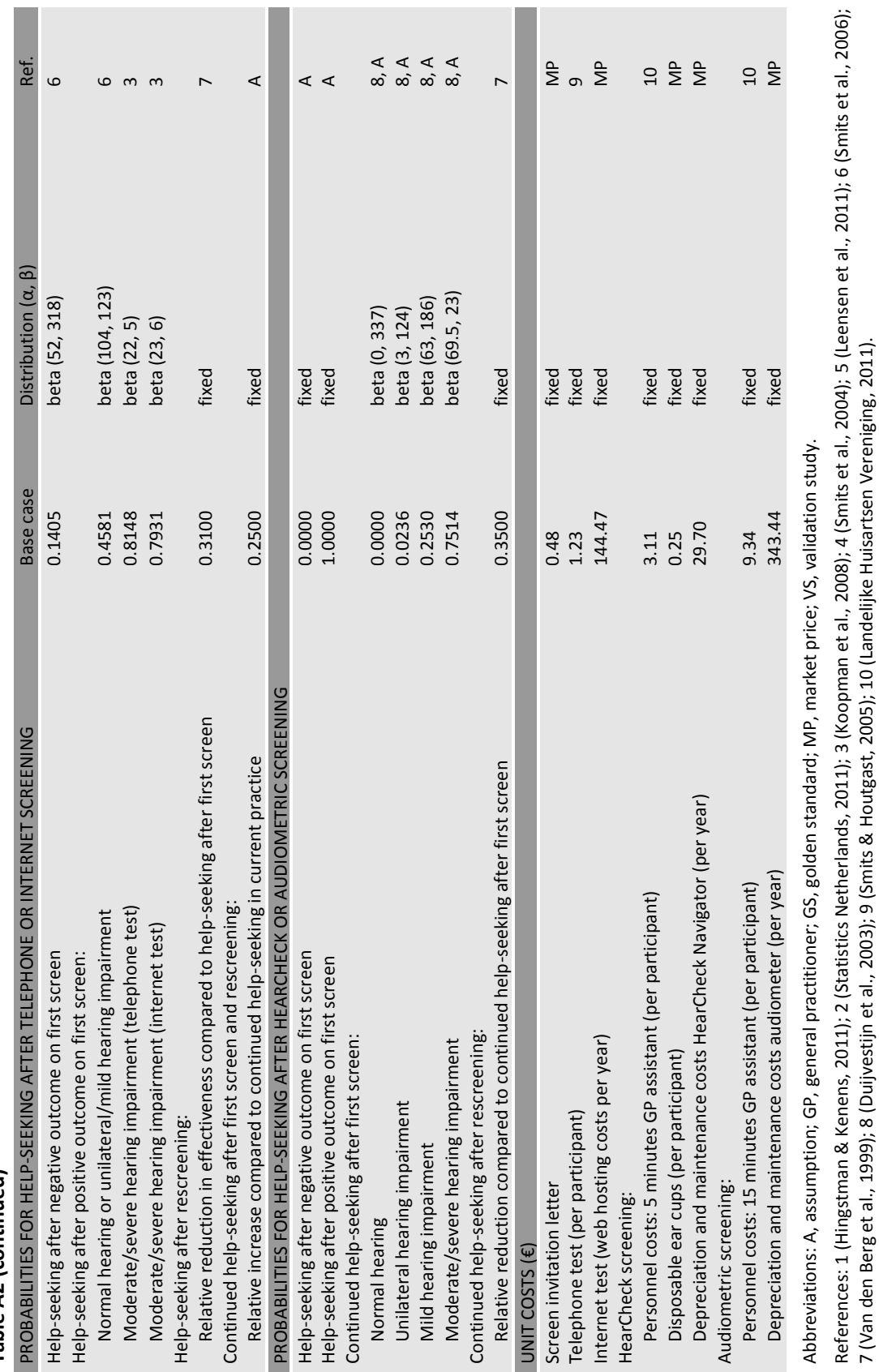




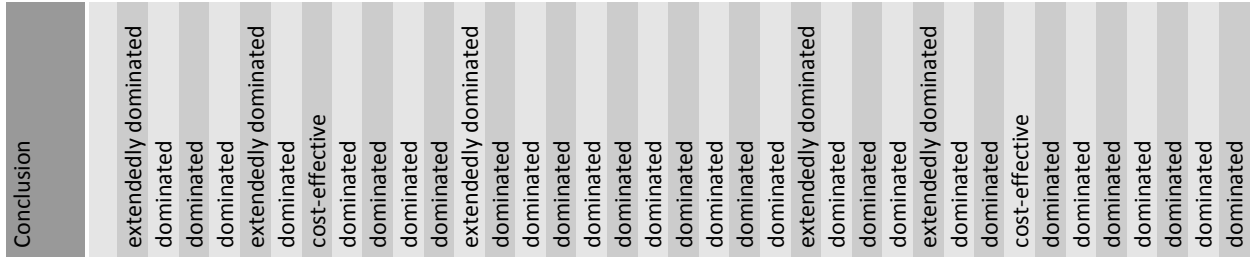

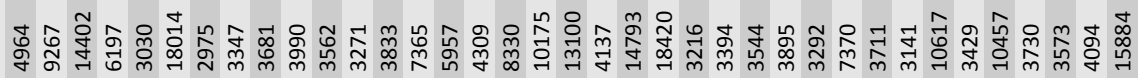

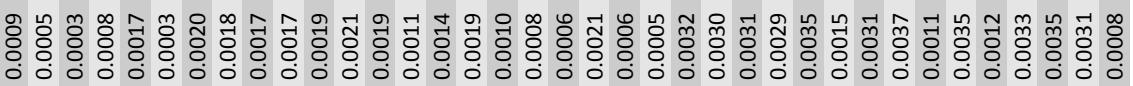

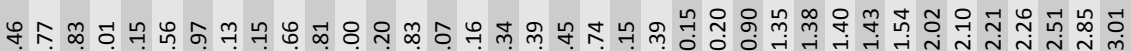

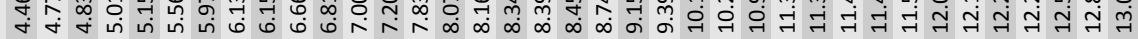

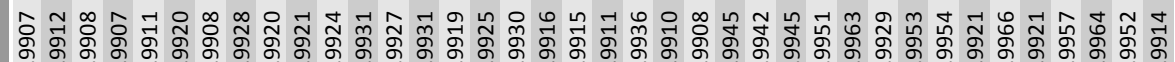

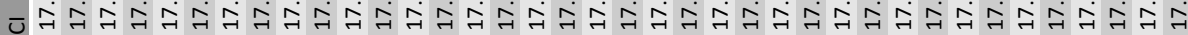
웅ㅇㅇㅇㅇㅇㅇㅇㅇㅇㅇㅇㅇㅇㅇㅇㅇㅇㅇㅇㅇㅇㅇㅇㅇㅇㅇㅇㅇㅇㅇㅇㅇㅇㅇㅇㅇㅇㅇㅇㅇㅇㅇㅇㅇㅇㅇㅇㅇㅇㅇㅇㅇㅇㅇㅇ은

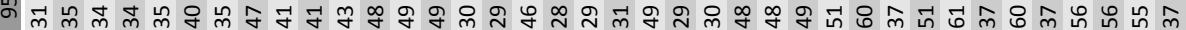
$\hat{m} \hat{m} \hat{m} \hat{m} \hat{m} \hat{m} \hat{m} \hat{m} \hat{m} \hat{m} \hat{m} \hat{m} \hat{m} \hat{m} \hat{m} \hat{m} \hat{m} \hat{m} \hat{m} \hat{m} \hat{m} \hat{m} \hat{m} \hat{m} \hat{m} \hat{m} \hat{m} \hat{m} \hat{m} \hat{m} \hat{m} \hat{m} \hat{m} \hat{m} \hat{m} \hat{m} \hat{m} \hat{m}$

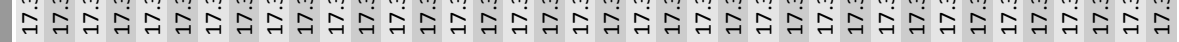

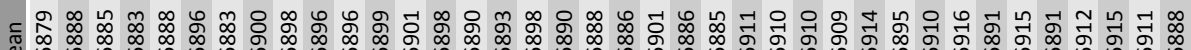

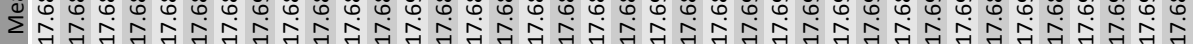

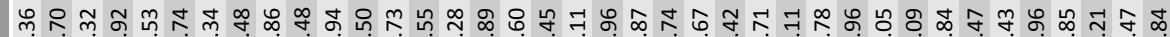
ஏ

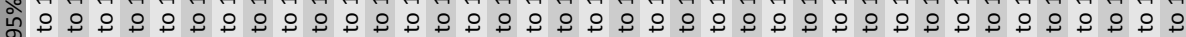

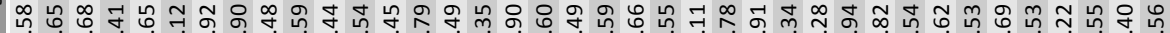

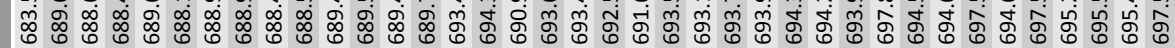

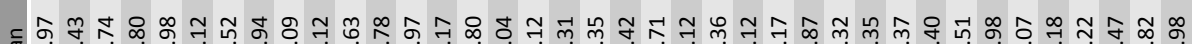

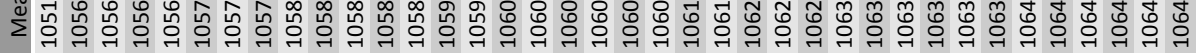

인 กิ ㅇำ 8

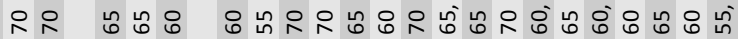

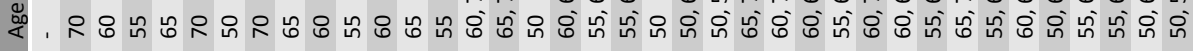
no

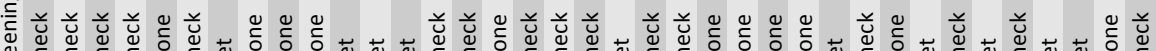

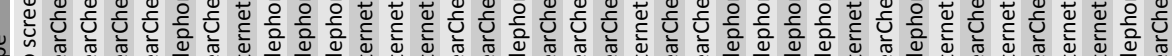

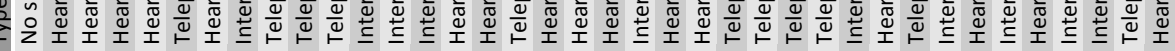




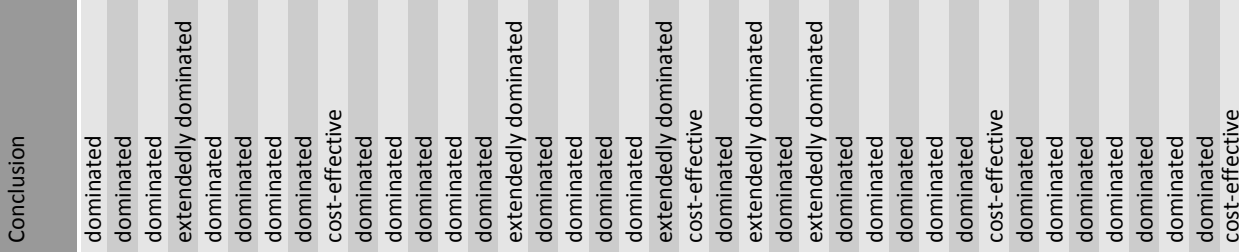

눙

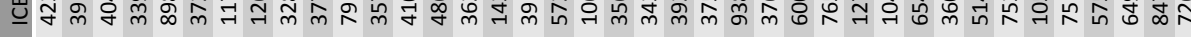

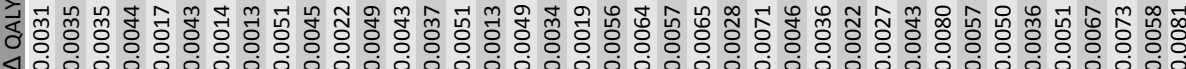

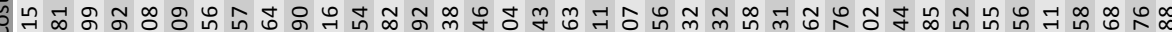

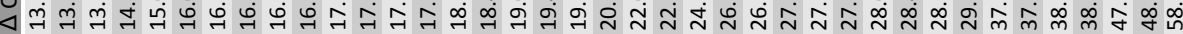

ఫ

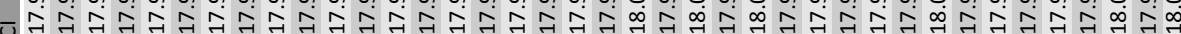

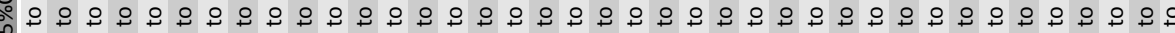

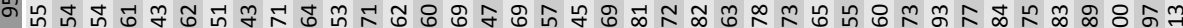
$\hat{m} \hat{m} \hat{m} \hat{m} \hat{m} \hat{m} \hat{m} \hat{m} \hat{m} \hat{m} \hat{m} \hat{m} \hat{m} \hat{m} \hat{m} \hat{m} \hat{m} \hat{m} \hat{m} \hat{m} \hat{m} \hat{m} \hat{m} \hat{m} \hat{m} \hat{m} \hat{m} \hat{m} \hat{m} \hat{m} \hat{m} \hat{m} \hat{m} \hat{m} \hat{m} \hat{m} m_{m}^{\infty} \hat{m}_{m}^{\infty}$

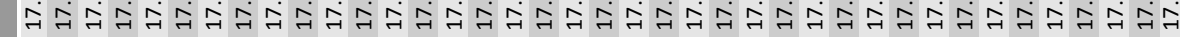

๘ન్ન

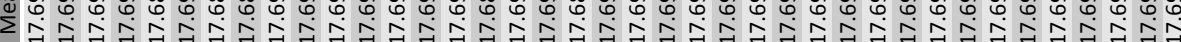

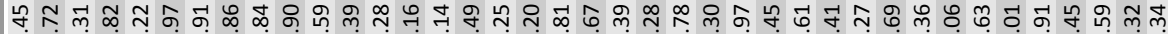

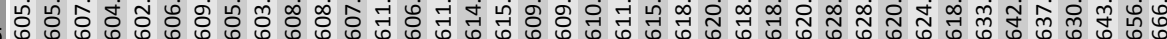
年

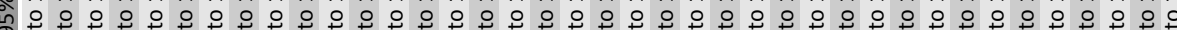

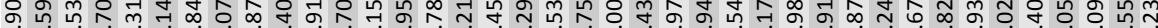

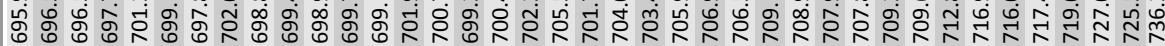

두 苑

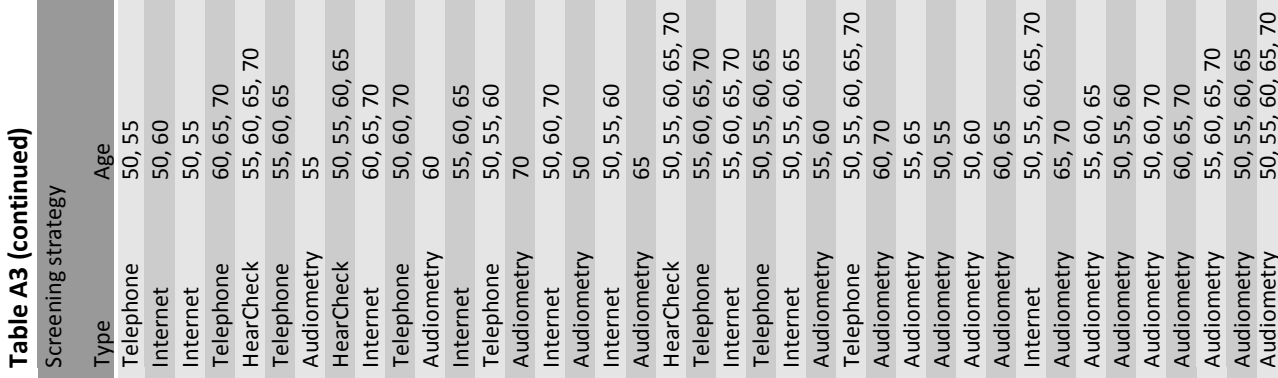




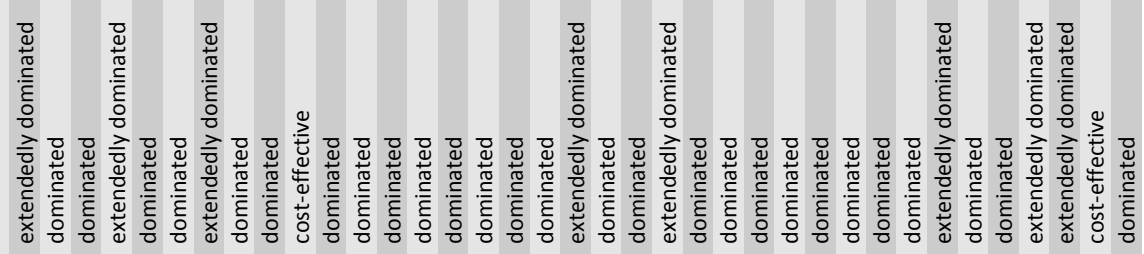

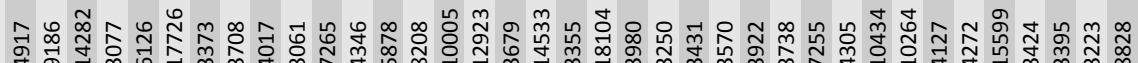

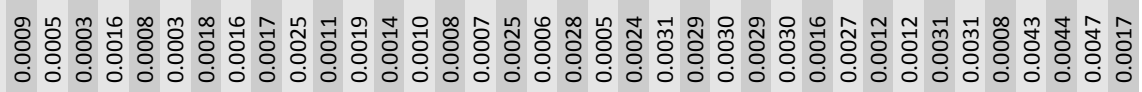
กู \&

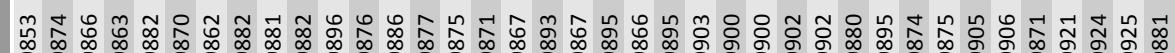

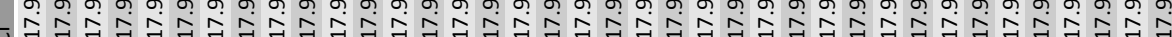

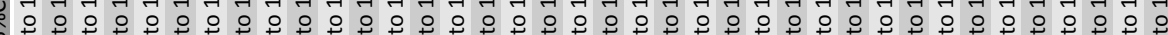
织 $\hat{m} \hat{m} \hat{m} \hat{m} \hat{m} \hat{m} \hat{m} \hat{m} \hat{m} \hat{m} \hat{m} \hat{m} \hat{m} \hat{m} \hat{m} \hat{m} \hat{m} \hat{m} \hat{m} \hat{m} \hat{m} \hat{m} \hat{m} \hat{m} \hat{m} \hat{m} \hat{m} \hat{m} \hat{m} \hat{m} \hat{m} m_{m}^{\infty} \hat{m} m_{m}^{\infty} m_{m}^{\infty} \hat{m}$

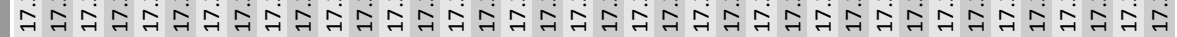

กน

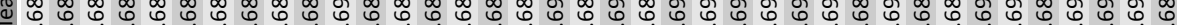
र

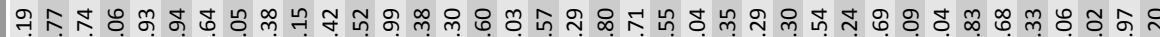

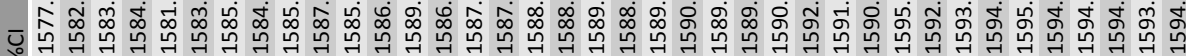

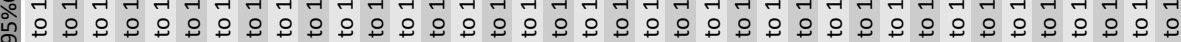

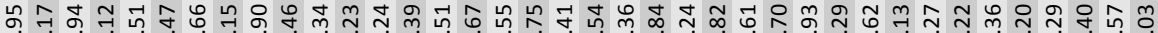
ڤo

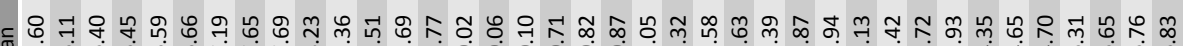

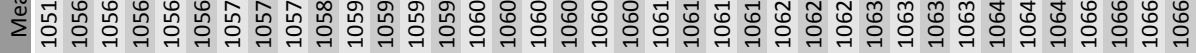

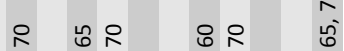
온

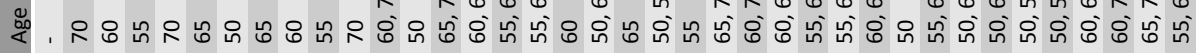
$\stackrel{10}{c}$

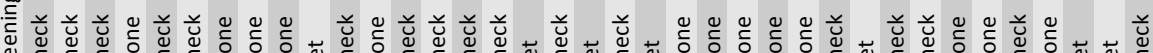

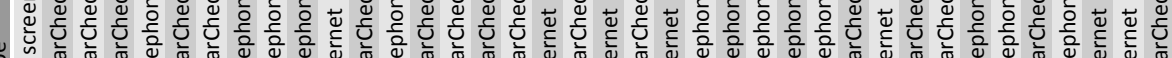

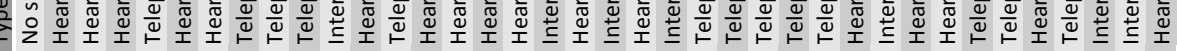




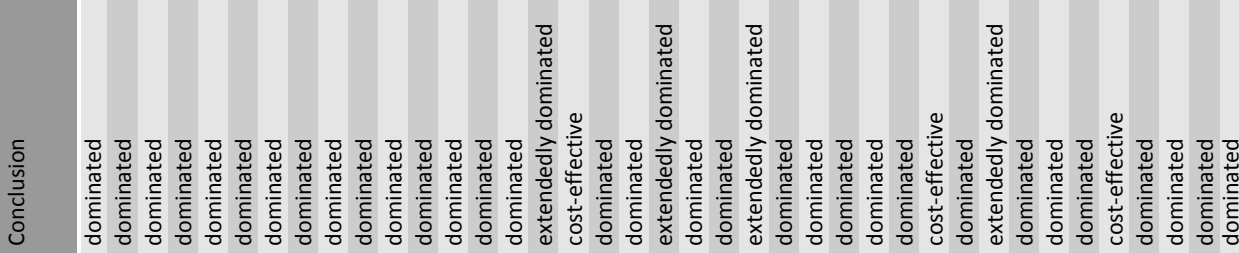

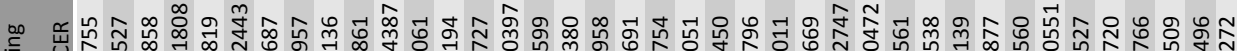

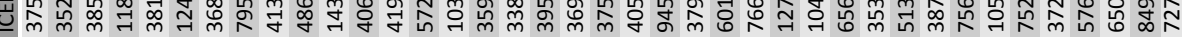

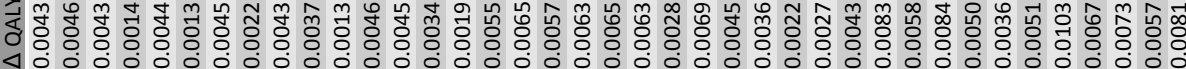

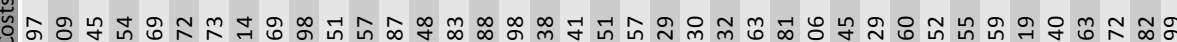
४

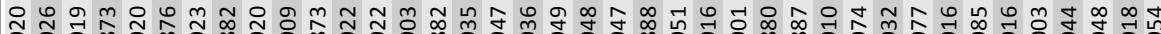
ুু व

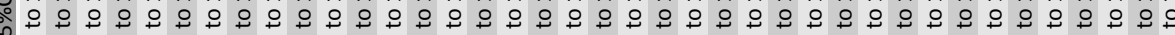

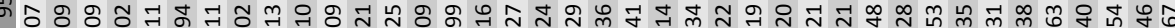

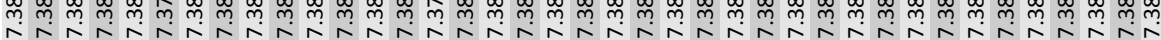

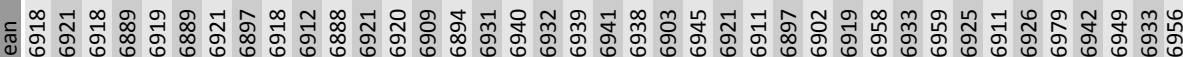
年

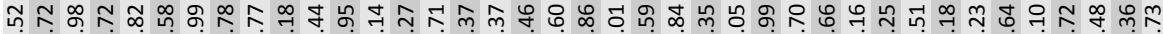

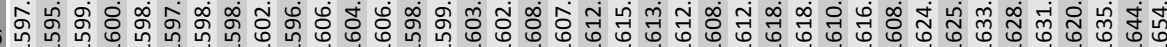

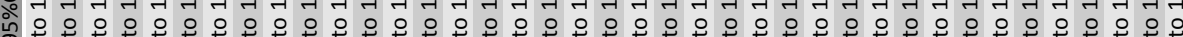

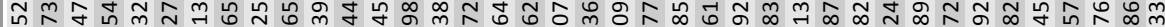

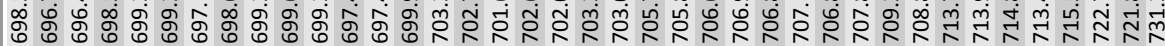

ᄃํํㅇํ ๆ 选过

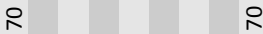

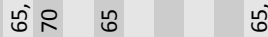

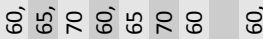

$\therefore \frac{1}{1}$ ㄴ. กู ที่ ㄴำ ํํ 8ิ
뇨 요

จิ่

○ in i

ถิ่ ष्้ 


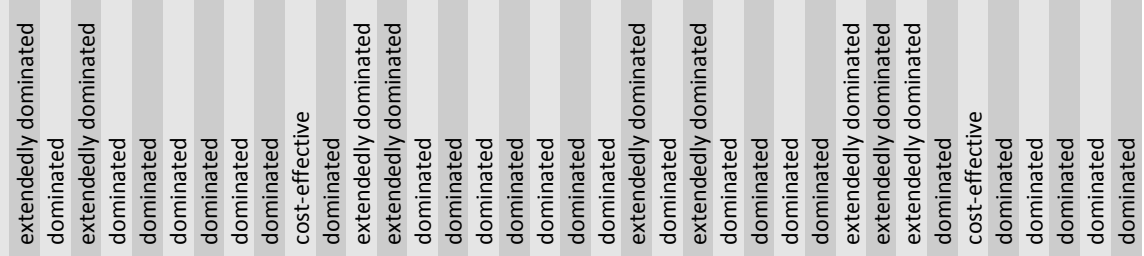

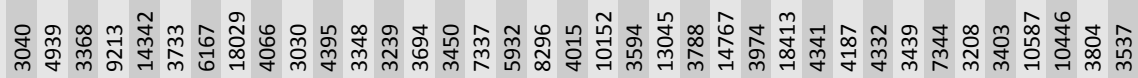

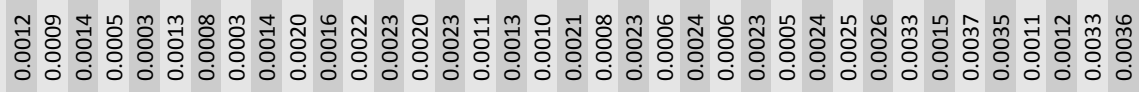
ำ ๆ ํํำ ณ

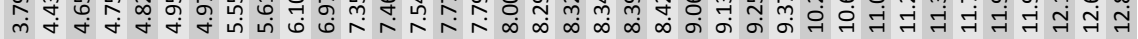

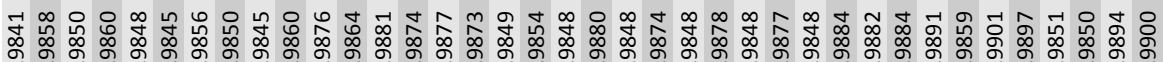

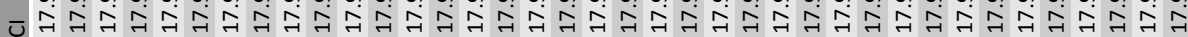
웅ㅇㅇㅇㅇㅇㅇㅇㅇㅇㅇㅇㅇㅇㅇㅇㅇㅇㅇㅇㅇㅇㅇㅇㅇㅇㅇㅇㅇㅇㅇㅇㅇㅇㅇㅇㅇㅇㅇㅇㅇㅇㅇㅇㅇㅇㅇㅇㅇㅇㅇㅢ

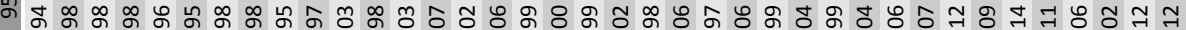

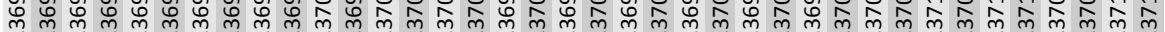

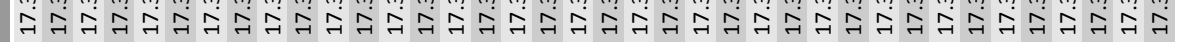

ᄃ $\begin{gathered}\hat{0} \\ \infty\end{gathered}$

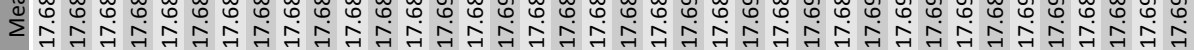

๒ ํำ

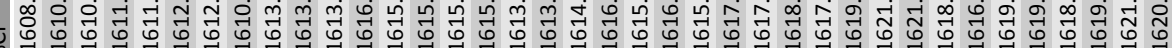

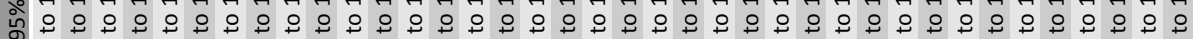
సิ

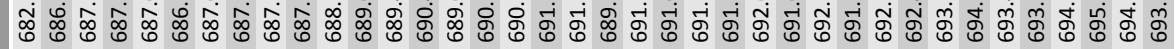

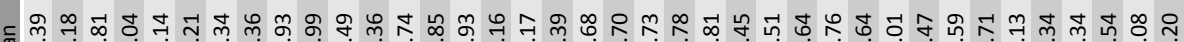

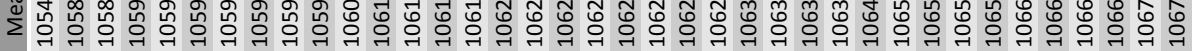

ㅇำ นำ

옹ำ

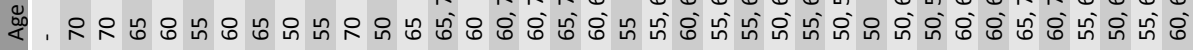
$\stackrel{\infty}{\stackrel{5}{=}}$

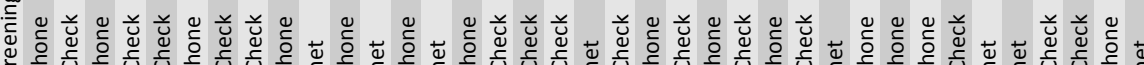
U

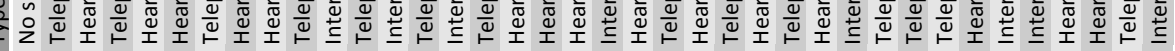




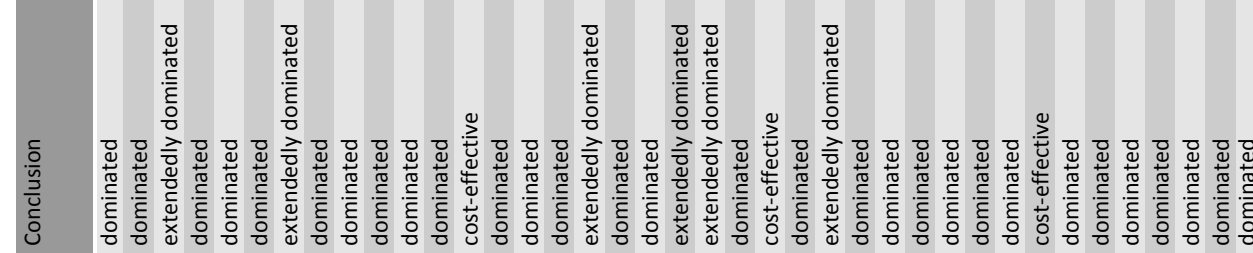

员

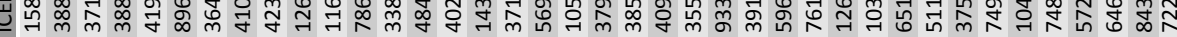

\section{خ 荌:

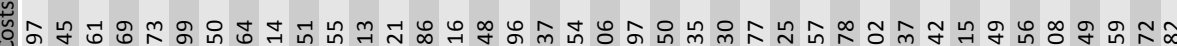

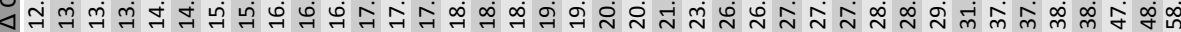

我 ळ 行 옹ㅇㅇㅇㅇㅇㅇㅇㅇㅇㅇㅇㅇㅇㅇㅇㅇㅇㅇㅇㅇㅇㅇㅇㅇㅇㅇㅇㅇㅇㅇㅇㅇㅇㅇㅇㅇㅇㅇㅇㅇㅇㅇㅇㅇㅇㅇㅇㅇㅇㅇㅇㅇ ๙ $\hat{m} \hat{m} \hat{m} \hat{m} \hat{m} \hat{m} \hat{m} \hat{m} \hat{m} \hat{m} \hat{m} \hat{m} \hat{m} \hat{m} \hat{m} \hat{m} \hat{m} \hat{m} \hat{m} \hat{m} \hat{m} \hat{m} \hat{m} \hat{m} \hat{m} \hat{m} \hat{m} \hat{m} \hat{m} \hat{m} \hat{m} \hat{m} \hat{m} \hat{m} \hat{m} \hat{m} \hat{m} \hat{m} \hat{m}$

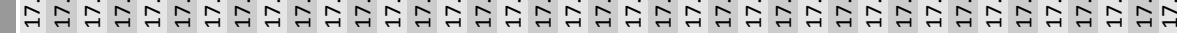

๘

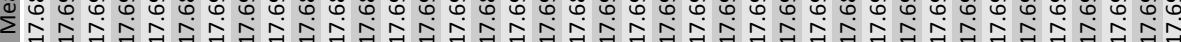

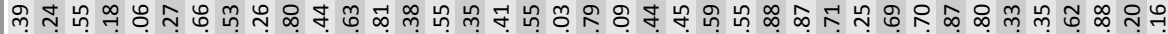
-

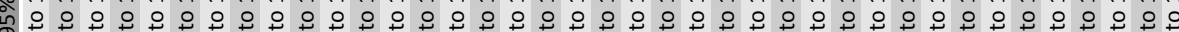

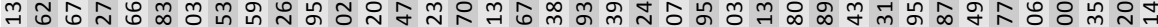
ด̆

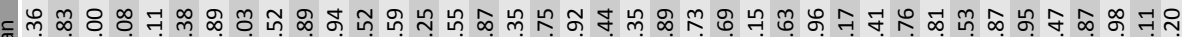

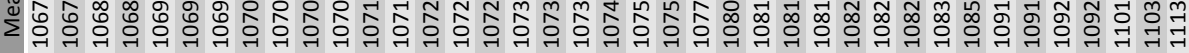
오인 นึ่ นึ่

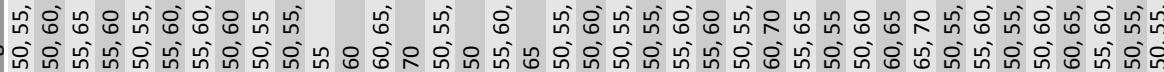

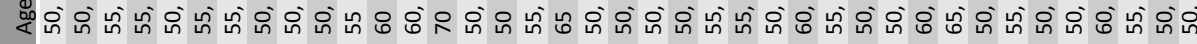

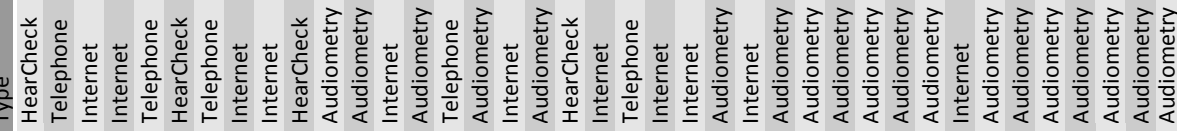




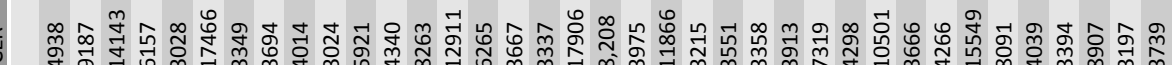
岁

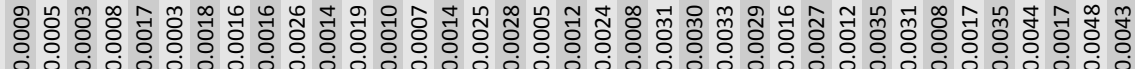

f

స -

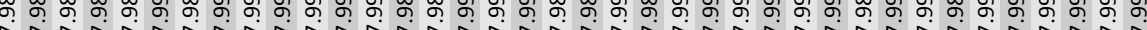

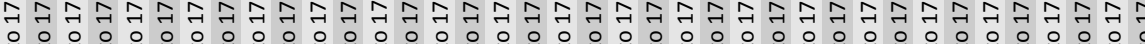

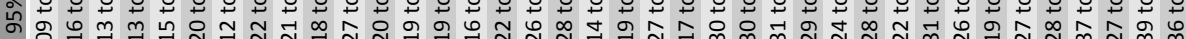

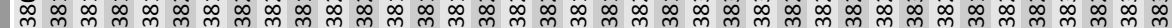

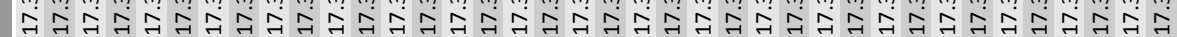

\section{ᄃำ}

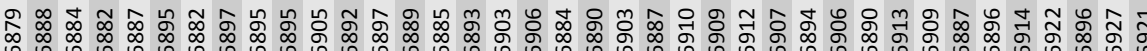

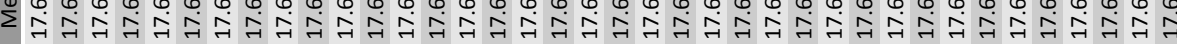
นุ ப.

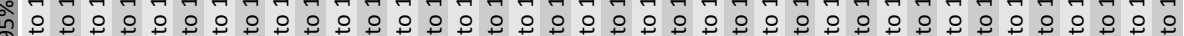

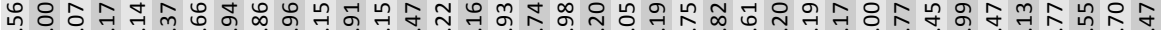

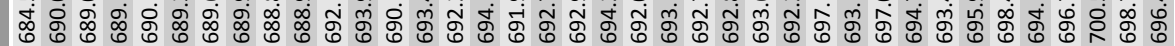

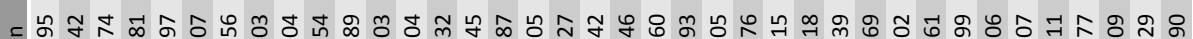
选苍全 


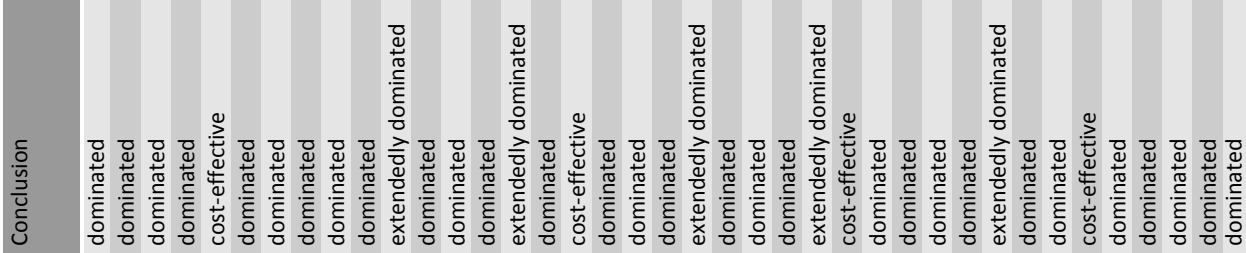

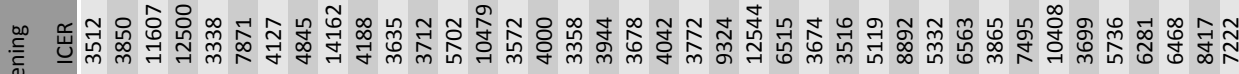

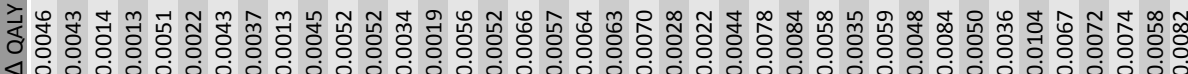

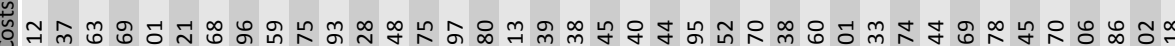 ব凷}

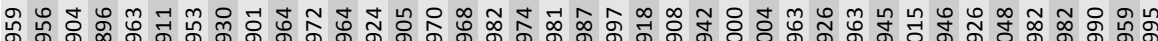
ুু ুळळ ু ু

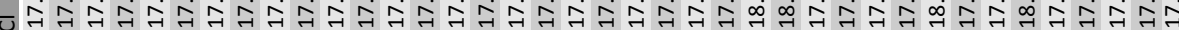

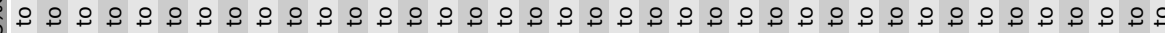

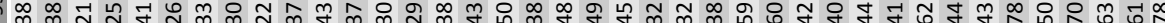

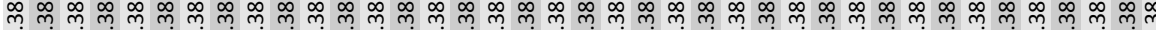

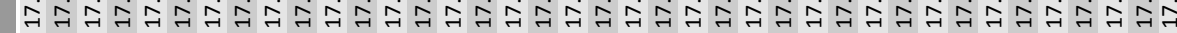

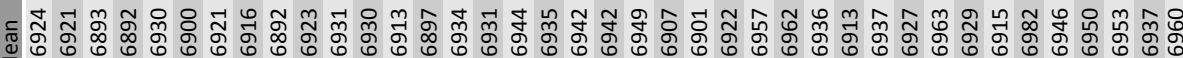
皮茫

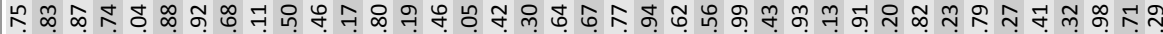

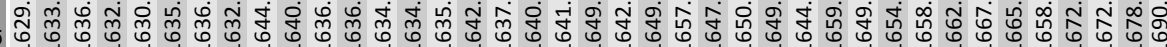

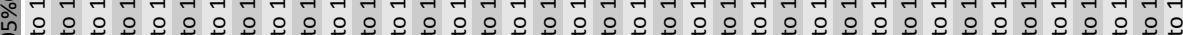

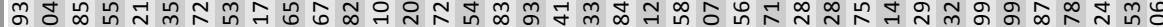

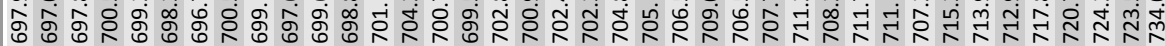

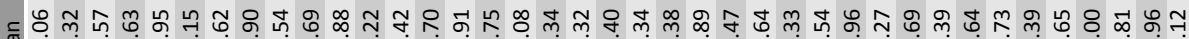

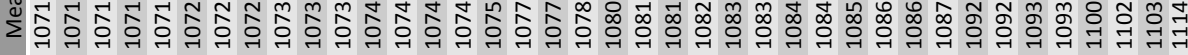

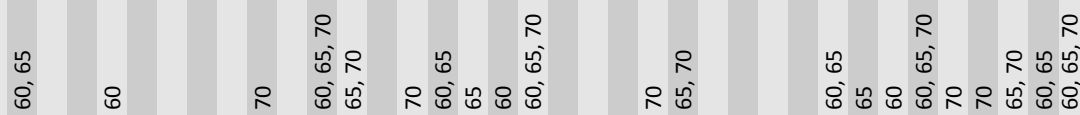

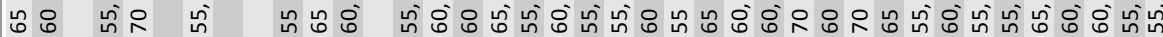
षั 


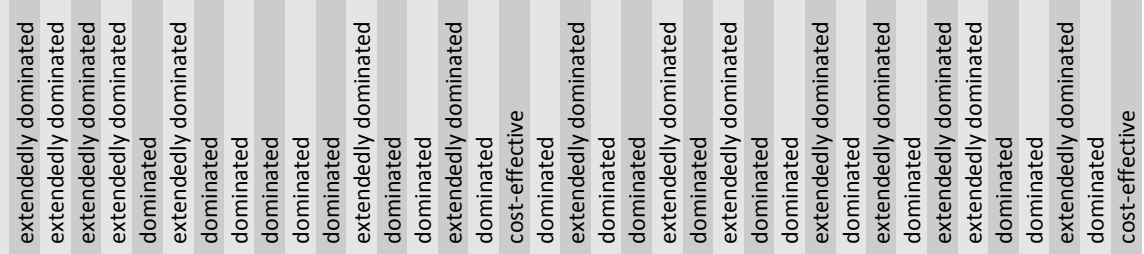

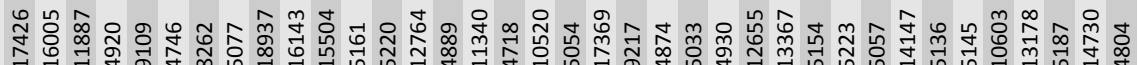

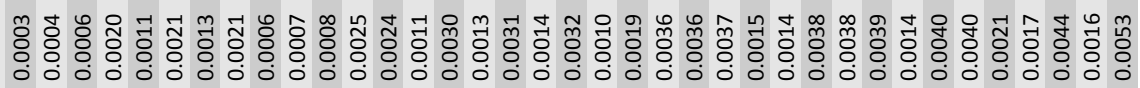

t 他

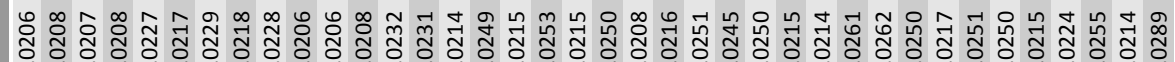
ธ 웅우웅ㅇㅇㅇㅇㅇㅇㅇㅇㅇㅇㅇㅇㅇㅇㅇㅇㅇㅇㅇㅇㅇㅇㅇㅇㅇㅇㅇㅇㅇㅇㅇㅇㅇㅇㅇㅇㅇㅇㅇㅇㅇㅇㅇㅇㅇㅇㅇㅇㅇㅇㅇㅇㅇㅇㅇㅇㅇ

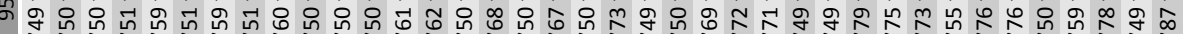

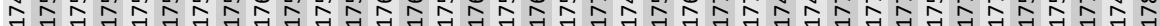

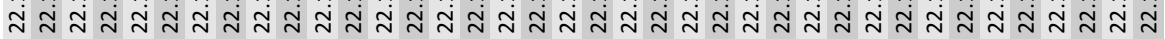

\section{듀}

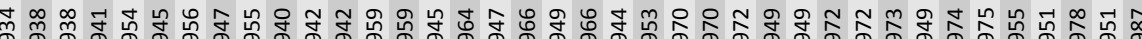
以n

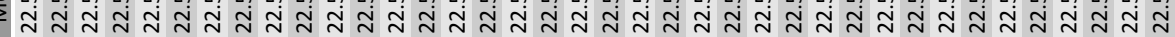

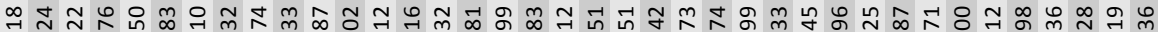

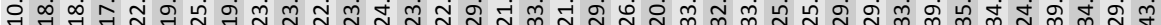

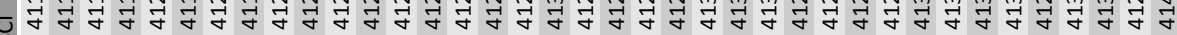
웅ㅇㅇㅇㅇㅇㅇㅇㅇㅇㅇㅇㅇㅇㅇㅇㅇㅇㅇㅇㅇㅇㅇㅇㅇㅇㅇㅇㅇㅇㅇㅇㅇㅇㅇㅇㅇㅇㅇㅇㅇㅇㅇㅇㅇㅇㅇㅇㅢ

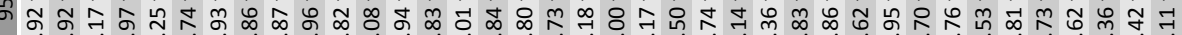
ڤ ळ

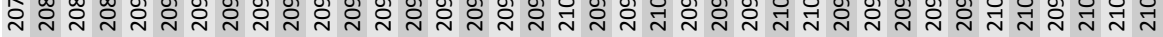

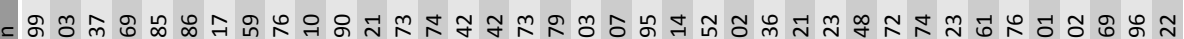

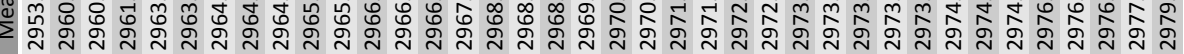

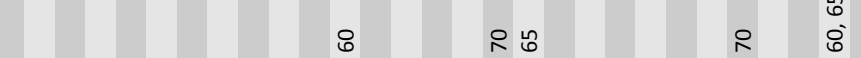

นnํㅇㅇㅛ ถ

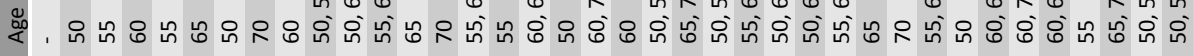

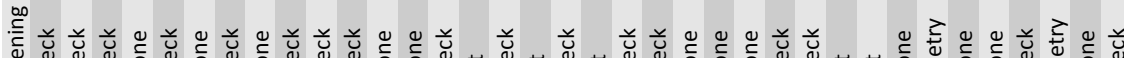

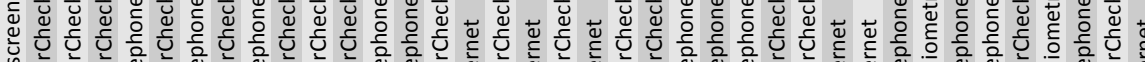
$\begin{array}{ll} & \\ 0 & \end{array}$ 


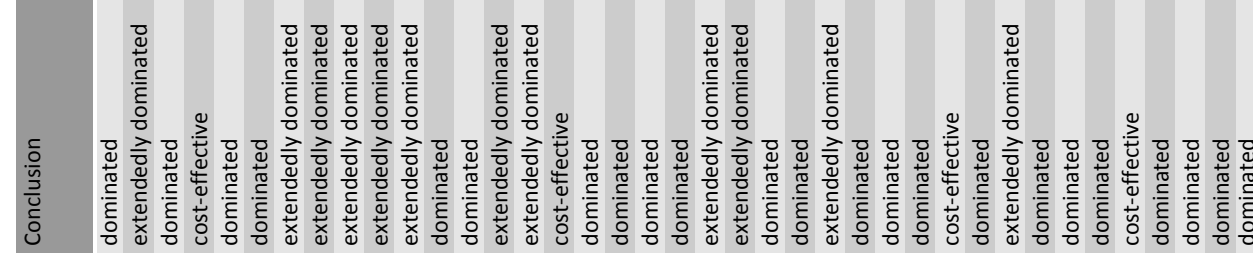

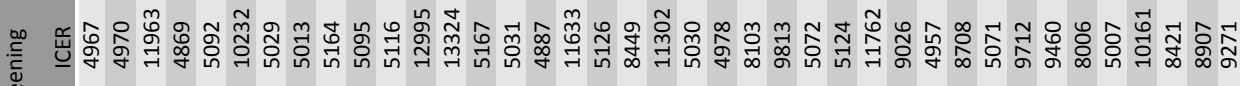

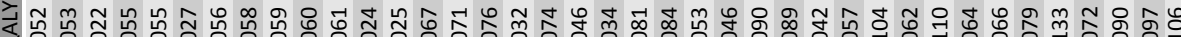

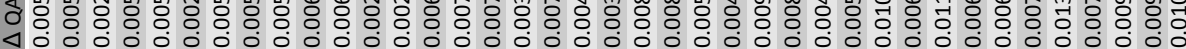 \\ 苟โิ

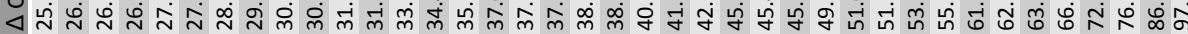

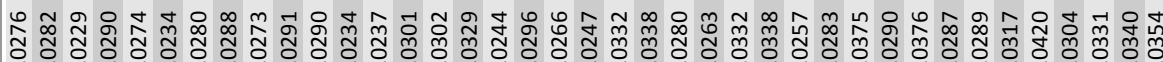

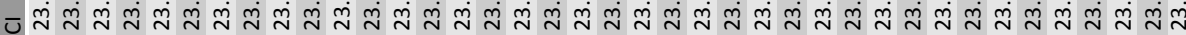
앙ㅇㅇㅇㅇㅇㅇㅇㅇㅇㅇㅇㅇㅇㅇㅇㅇㅇㅇㅇㅇㅇㅇㅇㅇㅇㅇㅇㅇㅇㅇㅇㅇㅇㅇㅇㅇㅇㅇㅇㅇㅇㅇㅇㅇㅇㅇㅇㅇㅇㅇㅇㅇㅇㅇㅇㅇㅇㅇㅇㅇㅇㅇ ת。

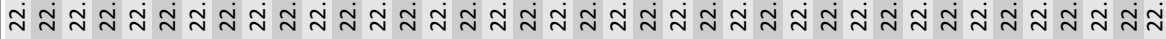

๘。

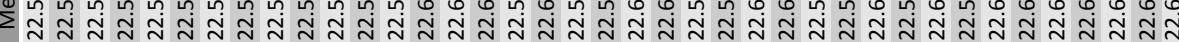

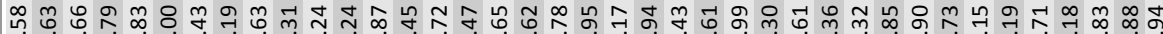

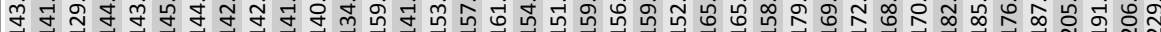

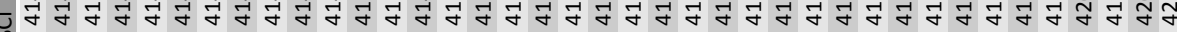

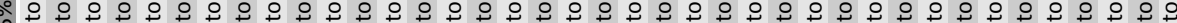
స

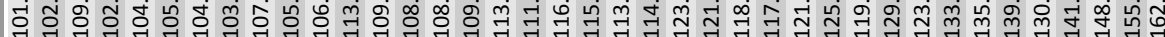

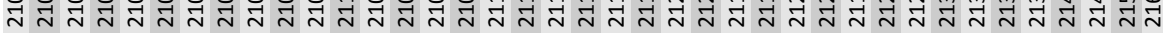

웅 도

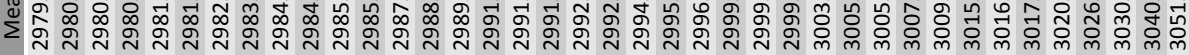

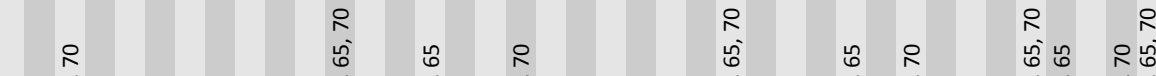
8 กิ

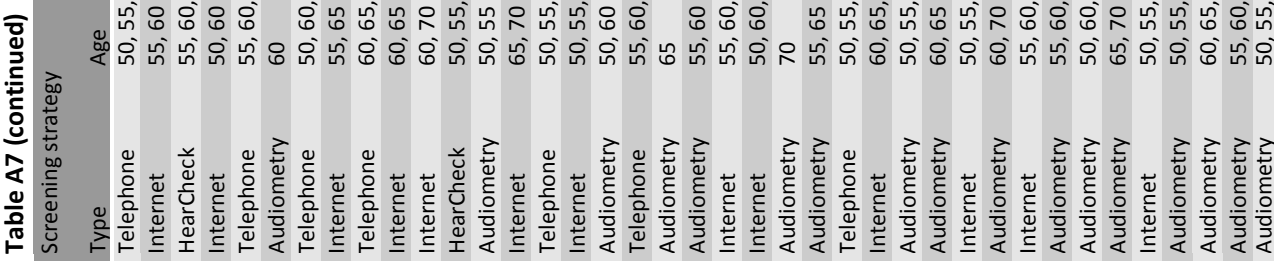




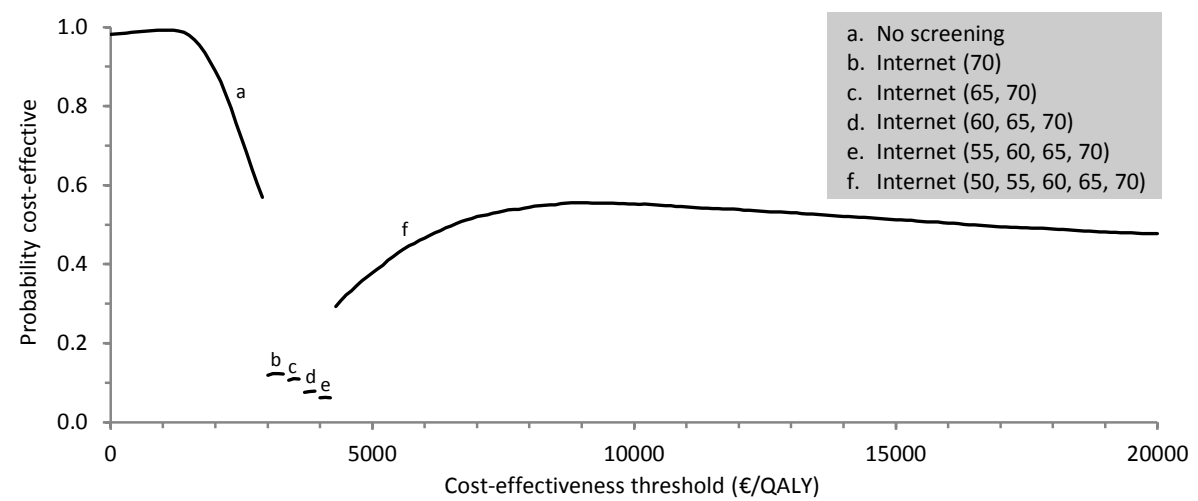

Figure A2. Cost-effectiveness acceptability frontier: results of one-way sensitivity analysis 1

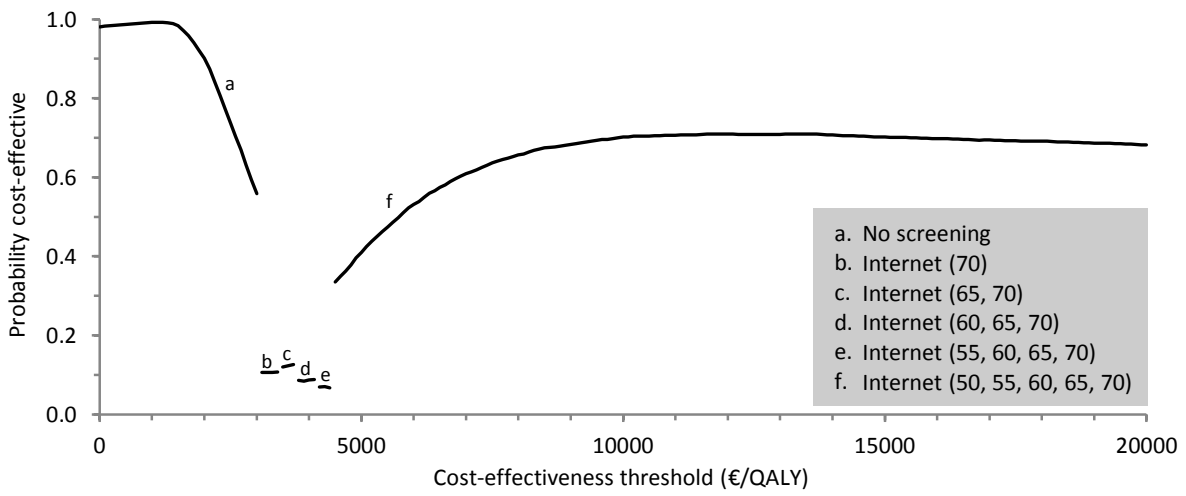

Figure A3. Cost-effectiveness acceptability frontier: results of one-way sensitivity analysis 2

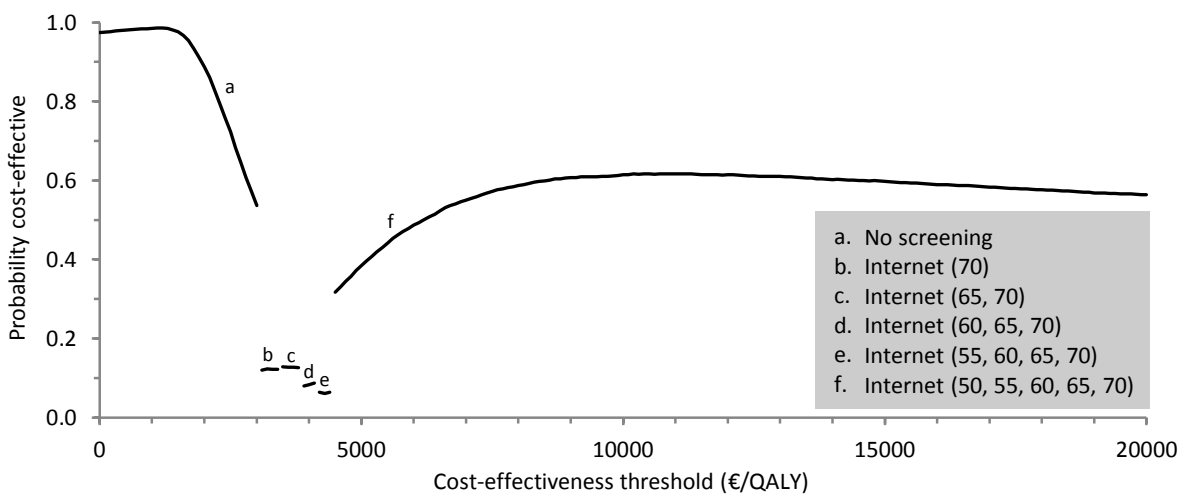

Figure A4. Cost-effectiveness acceptability frontier: results of one-way sensitivity analysis 3 


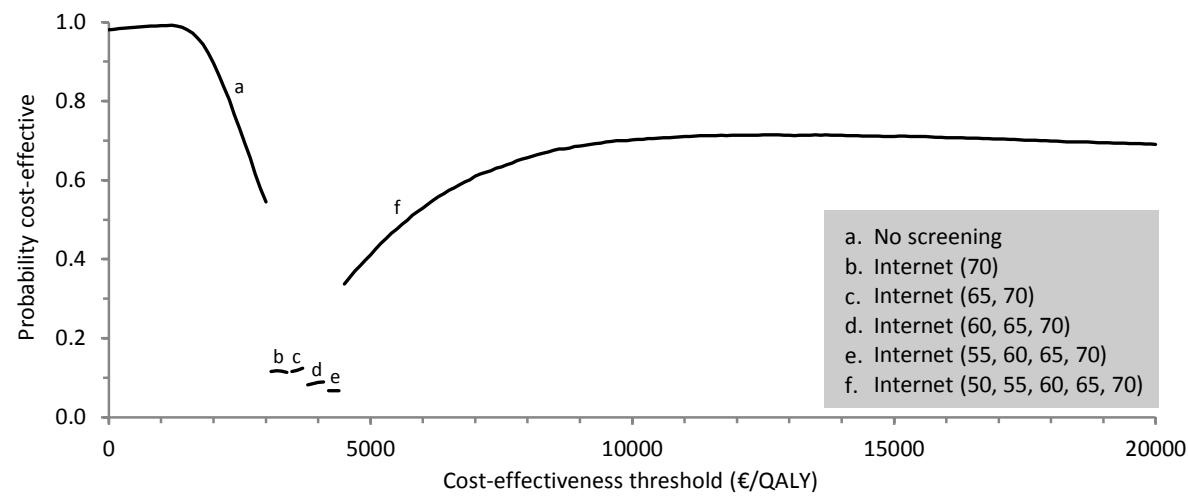

Figure A5. Cost-effectiveness acceptability frontier: results of one-way sensitivity analysis 4

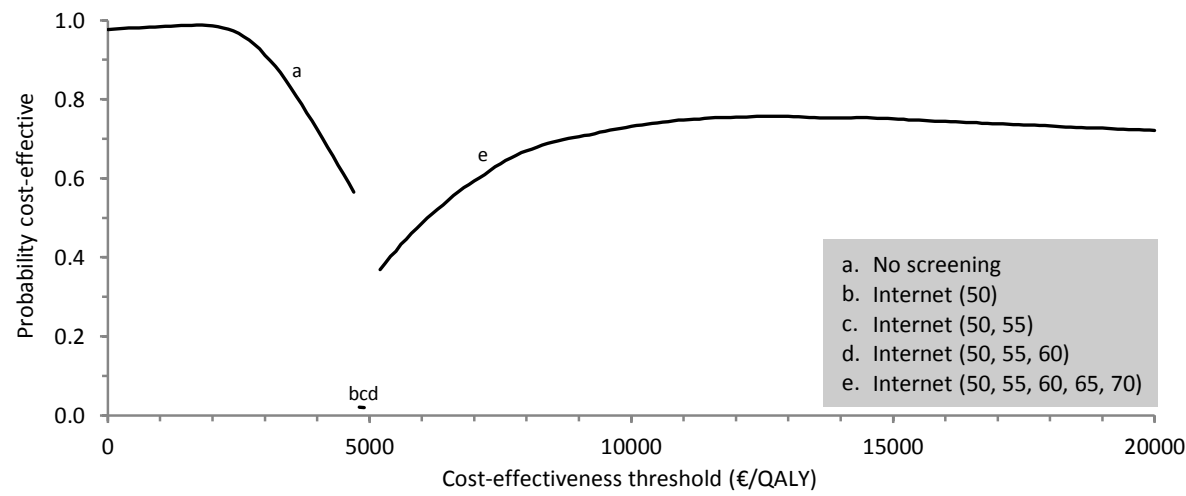

Figure A6. Cost-effectiveness acceptability frontier: results of one-way sensitivity analysis $\mathbf{5}$ 



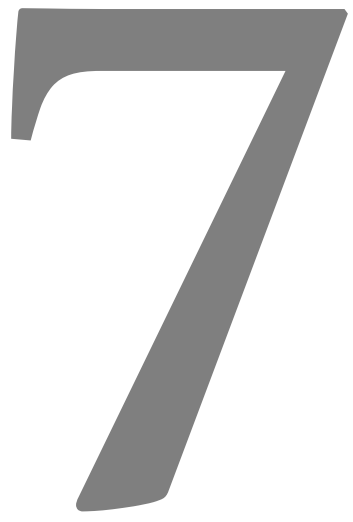

General discussion 

The objective of this thesis was to gain more insight into how to design an adult hearing screening programme for nationwide implementation in the Netherlands. In this chapter, the main findings with regard to the research questions are summarised, followed by a discussion about methodological issues. The chapter ends with a statement of our conclusions and recommendations.

\section{Main findings and implications}

\section{Minimising harms of screening}

Interviews with seven adults who screened positive for hearing impairment but who never or only occasionally had hearing complaints revealed that screening causes no or little harm (Chapter 2). The only indicator of harm in this study was that some participants felt indignant about the screen outcome because they felt they were being unfairly labelled as hearing-impaired. Expression of indignation was related to the people's beliefs with regard to the validity of the screening test. The screen outcome caused feelings of indignation only in people who judged the test validity negatively; people with a positive judgment on the test validity were more likely to accept the screen outcome. This suggests that, to minimise the harms and maximise the effectiveness of screening, effort should be put in convincing screen participants that the test is valid. The study showed that one may deal with screen participants' concerns on the test validity by giving a clear explanation of how the test works, by offering the screening in a silent environment without distracting factors, or by offering immediate rescreening (using either the same or another screening test).

The screening also evoked surprise, disbelief, incomprehension, disappointment, concern, emotional pain, and sadness. These emotions were similar to the emotions reported by people diagnosed with a hearing impairment based on standard clinical diagnostic tests (Martin et al., 1989). Studies have shown that these emotions are part of the natural response to an increasing awareness of hearing loss (Engelund, 2006; Hindhede, 2012; Wallhagen, 2010; Weinberger, 1980). They might therefore be viewed as triggered but not caused by the screening. The fact that screening evoked such emotions supports the notion that screening can incite or accelerate the hearing loss recognition process and indirectly motivate people to seek help earlier. The feelings of emotional pain and sadness probably reflect harm resulting from an increasing awareness of hearing loss, not from the screening itself. Even without the screening, part of the participants would probably have experienced these emotions at a certain point during the hearing loss recognition process. The screening made it happen earlier and probably more abruptly. 


\section{Preventing non-use of dispensed hearing aids}

\section{Impact of non-use of dispensed hearing aids}

When analysing interviews with adults who owned but did not use hearing aids (Chapter 3), three types of non-users were distinguished: non-users with no or mild hearing handicap ('Type A non-users'), non-users with moderate to severe hearing handicap who attributed the non-use to internal factors ('Type B non-users'), and nonusers with moderate to severe hearing handicap who attributed the non-use to external factors ('Type $C$ non-users'). Type A non-users were indifferent about the nonuse; Type B non-users expressed feelings of self-annoyance, self-disappointment, and self-blame; and Type C non-users expressed feelings of frustration, indignation, sadness, anger, powerlessness, and hopelessness. Disapproval of the non-use by significant others led to feelings of shame and guilt, but only for Type B non-users. The study showed that non-use of dispensed hearing aids can cause (emotional) harm, which underscores the importance of non-use prevention.

The impact of non-use of dispensed hearing aids on the effectiveness of adult hearing screening can be analysed with the state transition model described in Chapter 6 . The annual probability of hearing aid discard was initially set at 0.06 , based on a study by Van den Brink and colleagues (1996). The best screening strategy (i.e. internet screening at age 50, 55, 60, 65, and 70) led on average to 0.0104 more quality-adjusted life years (QALYs) per person than no screening. If the hearing aid discard probability had been set at 0.00 , the screening strategy would have led to 0.0159 more QALYs per person than no screening (post-hoc analysis, not shown before). Thus, non-use of dispensed hearing aids reduces the effectiveness of adult hearing screening with approximately $35 \%$.

\section{Prevention strategies}

The interview study presented in Chapter 3 gave two directions for prevention of nonuse of dispensed hearing aids. Firstly, non-use may partly be prevented by more careful consideration whether hearing aid fitting will be effective in an individual. Although hearing aid fitting is the conventional and generally most effective rehabilitation option for hearing loss, some people may benefit more from alternatives, like communication training, assistive listening devices or doing nothing. Hearing care professionals should inform hearing help-seekers about all available rehabilitation options and decide together about the best option. In particular, the perceived severity of the hearing handicap, not the audiograms, should guide this shared decision making process. For adults who perceive no or only mild hearing handicap, doing nothing is probably the best option. Furthermore, uncontrollable (external) factors that may limit hearing aid benefit should be identified before or during a hearing aid trial and should be considered as contraindications for hearing aid purchase. For example, if someone primarily wants to improve his or her ability to understand speech in a noisy working environment, the person may benefit more from training in speech reading than from hearing aids. 
A second important strategy to prevent non-use of dispensed hearing aids is by offering comprehensive counselling and training during the hearing aid trial. Boas and colleagues (2001) found that a counselling programme was a cost-effective strategy to decrease non-use among new hearing aid owners. Our study identified two topics that should definitely be addressed in a counselling programme. First, the programme should address the limitations of hearing aids. People should be assisted to understand and accept the hearing aids' limitations. Secondly, the counselling programme should address hearing aid self-efficacy: people should become confident that they are able to become successful hearing aid users. Someone's hearing aid self-efficacy can be measured at the time of hearing aid fitting, using the MARS-HA (measure of audiologic rehabilitation self-efficacy for hearing aids) questionnaire developed by West and Smith (2007). Knowing someone's level of hearing aid self-efficacy can assist the hearing care professional in determining whether or not the person needs intensive counselling or self-efficacy training during the hearing aid trial in order to become a successful hearing aid user. By building hearing aid self-efficacy, adults will be more motivated to adjust to hearing aids, persevere when hearing aid difficulties arise, and apply more effort toward successful hearing aid use. Smith and West (2006) and Meyer and colleagues (2014) described a number of strategies for enhancing hearing aid self-efficacy, including role play and motivational coaching by hearing care professionals or significant others. People who are still dissatisfied with a hearing aid at the end of the trial should be not be encouraged to purchase the hearing aid.

\section{Target population}

\section{Residents of care homes for the elderly}

Chapter 4 describes the effects and costs of a hearing screening and rehabilitation programme that was organised in eight residential care homes for the elderly in the Netherlands. We hypothesised that easy accessibility of hearing care professionals would be a great facilitator for help-seeking and hearing aid uptake. Of the 522 residents without hearing aids at the start of the programme, $29(6 \%)$ owned hearing aids at the end. The programme costs per new hearing aid owner were $€ 2552$. Considering that the costs of successful hearing aid trials and hearing aids together accounted for $64 \%$ of the programme costs, the costs per new hearing aid owner seem reasonable. However, when deciding on implementation of the programme, one should keep in mind that the residents' time to benefit from hearing aids is limited because their expected survival is only two years (ActiZ, 2010). Besides, hearing aid ownership does not necessarily mean that the hearing aid is used and that it reduces the hearing handicap and improves the residents' quality of life. We were unable to measure these outcomes but from a study by Gussekloo and colleagues (2003) to the effectiveness of a screening and rehabilitation programme targeted at 85-year-old adults we know that only $40 \%$ of the new hearing aid owners were using the hearing aid on regular basis one year after the programme.

Hearing aid ownership increased only from $28 \%$ at the start of the programme to $33 \%$ at the end. The low hearing aid uptake during the programme indicates that most of 
the oldest elderly without hearing aids do not choose for hearing rehabilitation by hearing aid fitting, not even when possible mobility barriers are eliminated. Gussekloo and colleagues (2003), who came to a similar conclusion, reported that the major reason for not starting rehabilitation was that most elderly were at the same time concerned with trying to improve other aspects of their functioning which they perceived as more problematic than their hearing loss. This is probably also the reason for the low programme effectiveness in our study since care homes typically house frail elderly suffering from multiple comorbidities (ActiZ, 2010). Given the limited increase in hearing aid ownership and the high probability of hearing aid discard, we recommend against implementation of a screening and rehabilitation programme in residential care homes for the elderly.

Approximately one-fifth of the residents who already owned hearing aids were interested in consulting a hearing care professional and two-thirds of them had their hearing aids adjusted or had purchased one or two new hearing aids during the programme. These numbers reflect a high level of unmet need for aftercare. Apparently, many of the hearing aid owning residents were not fully satisfied with their hearing aids but had not taken the initiative to return to their hearing aid dispenser. Future research to strategies to address the unmet need for aftercare is recommended.

\section{Targets for high-risk group screening and optimal target age}

Chapter 5 reports on an observational study to predictors of hearing acuity aimed to identify potential targets for high-risk group screening. Predictors included in this study were age, gender, type of occupation, educational level, cardiovascular disease, diabetes, systemic inflammatory disease, hypertension, obesity, waist circumference, smoking, and physical activity level. Cross-sectional as well as longitudinal regression analyses were performed. In the age-stratified regression models, only $11 \%$ to $21 \%$ of the interindividual variance in hearing thresholds was explained by the non-audiometric predictors, which suggests that interindividual differences in hearing thresholds are primarily attributed to genetic variation. This notion is supported by the fact that the hearing deterioration rate showed a strong positive correlation with the baseline hearing thresholds and by two recent twin studies (Viljanen et al., 2007; Wingfield et al., 2007). Given the large role of genetics in the development and progression of hearing loss, it may be interesting to target screening at people who have a family history of hearing loss. We were not able to include information on family history in the models to assess whether people with hearing-impaired (first-degree) family members would form a suitable target for screening indeed. However, McMahon and colleagues (2008) found that people whose parents and/or siblings had a hearing loss were more likely to have a hearing loss themselves than people whose parents and siblings had a normal hearing. A difficulty with targeting a screening programme at people with a family history of hearing loss is that it is generally unknown who belongs to the target population. Family history of hearing loss is not reported in national or municipal registries, nor in medical dossiers of general practitioners. Besides, people 
might not know if hearing loss runs in their family when their parents died before the typical age of onset of age-related hearing loss and if they have no (older) siblings. Instead of trying to find members of the high-risk group and offer them screening, it might be easier to recommend people with known hearing loss to make their children or siblings aware of their increased risk for hearing loss.

The predictors that made a statistically significant contribution to the explanation of the interindividual variance in baseline and future hearing thresholds were higher age, male gender, manual occupation, and large waist circumference. The effects of the last two predictors were very small which makes them unsuitable as a base for target selection. The mean hearing thresholds of middle-aged and older men were found to be comparable to those of women who were approximately 8 to 9 years older, therefore, offering screening to women at a later age than to men may be considered in order to improve the cost-effectiveness of screening.

The observational study (Chapter 5 ) gave insight in the relationship between age and hearing thresholds but did not directly provide information on the optimal age for screening. To determine the optimal target age, we examined the effect of target age on the cost-effectiveness ratio of screening using a state transition model (Chapter 6). The effect of offering screening later to women than to men could unfortunately not be assessed with this Markov model because gender-specific data on help-seeking and hearing aid uptake was unavailable. The model was used to calculate the costs per QALY of current practice (no screening) and 76 adult hearing screening strategies. The screening strategies varied in the age at first screening (either 50,55, 60, 65, or 70 years), the number of repeated screenings (up to five repetitions), the time interval between repeated screenings (either 5 or 10 years), and the type of screening (four types). In the Netherlands, screening is deemed cost-effective and may be considered for nationwide implementation if the costs are below $€ 20000$ per QALY (Van den Berg et al., 2008). We found that all screening strategies were cost-effective compared to current practice. For each type of screening, the most cost-effective strategy was targeting the screening at people aged 50 with rescreening at ages $55,60,65$, and 70.

\section{Type of screening}

\section{Screening for ear age}

The qualitative study on adults' reactions on screening (Chapter 2) is the first study ever in which people were screened for ear age. We expected that informing people with a hearing loss about their ear age would be more effective in stimulating them to seek help and start using hearing aids than showing them an audiogram. This hypothesis was based on a study by Soureti and colleagues (2010) who found that people were more likely to make lifestyle changes when told what their heart age was than when given a risk percentage for cardiovascular disease. Calculation of the ear age was based on a comparison between measured hearing thresholds and age-specific population norms (Spoor et al., 1969). People with an ear age of 53 or lower were informed that their hearing was good; people with an ear age between 58 and 65 that their hearing 
was sufficient; people with an ear age between 68 and 74 that they probably had a hearing loss; and people with an ear age of 77 or higher that they had a hearing loss. People with an ear age of 68 or higher were advised to consider having extensive diagnostic testing. The study participants regarded the discrepancy between their ear age and chronological age as a more valuable indicator of the severity of their hearing loss than the printed advice and audiogram. The ear age had a strong emotional impact on people whose chronological age was much lower than their ear age. Although the results did not prompt them to start using hearing aids immediately after the screening, because they felt they were too young, being informed about their ear age might help the hearing loss recognition and acknowledgement process. Presenting the screen outcome using the ear age was not suitable for adults aged 65 years or older because their ear age may turn out to be almost equal to or lower than their calendar age, even if they have a hearing impairment. Since the ear age tells those people their hearing is normal for their age, it may demotivate them to seek help. Using the ear age is therefore only recommended when the screening is targeted at adults who are younger than 65 years.

\section{Internet screening, telephone screening, or opportunistic screening at the GP practice}

The state transition model (Chapter 6) was used to examine the cost-effectiveness of current practice (no screening), telephone screening, internet screening, screening at the GP practice with a hand-held screening device (Siemens HearCheck Navigator), and screening at the GP practice with a standard audiometer. The two screenings at the GP practice were opportunistic strategies since the screenings were offered to people at the moment they visited the GP practice for whatever reason. For the telephone and internet screening, on the other hand, all adults from a certain age were invited by letter. We found that all types of screening were cost-effective compared to current practice. Opportunistic screening at the GP practice was generally more costly and less effective than telephone or internet screening. Internet screening was slightly more cost-effective than telephone screening. Internet screening at age 50 , repeated at ages $55,60,65$, and 70 was the most cost-effective strategy, costing $€ 3699$ per QALY. At a threshold of $€ 20000$ per QALY this strategy had a probability of $100 \%$ to be costeffective compared to current practice and a probability of $69 \%$ to be more costeffective than any other strategy.

The internet test used in this study was the National Hearing Test (www.hoortest.nl) that was developed by Smits and colleagues (2006). It is a non-commercial fully automatic adaptive speech-in-noise test that uses digit-triplets as speech material. The test is highly accurate (Leensen et al., 2011). From the qualitative study on adults' reactions to screening (Chapter 2) we know that, for a screening to be effective and harmless, screen participants should be convinced that the test is valid. Some people may have doubts about the validity of the internet test, for example because they do not understand how the volume level of the computer boxes does not influence the test outcome. Such predictable concerns should be addressed in the screen invitation letter or on the website before the test starts. Advantages of the internet test with regard to 
the test validity are that people can control the test environment (by removing distracting factors like environmental noise) and that they can redo the test as often as needed for them to believe the outcome. Other advantages of the internet test are that people do not have to leave their house for testing and that they can do the test at any time that suits them. Internet access hardly forms a barrier for internet screening nowadays in the Netherlands as $98 \%$ of adults aged between 45 and 55 years, $94 \%$ of adults aged between 55 and 65 years, and $85 \%$ of adults aged between 65 and 75 years have access to internet at their home (Statistics Netherlands, 2013). Mentioning the telephone version of the National Hearing Test in the invitation letter for internet screening should be considered to make screening accessible for adults without internet access as well.

\section{Methodological issues}

\section{Objective versus subjective screen instrument}

A recent review of factors that influence help-seeking and hearing aid uptake concluded that both are more strongly related to perceived complaints than to audiometric hearing thresholds (Meyer \& Hickson, 2012). This suggests that a subjective screen instrument (e.g. questionnaire on hearing disability or handicap) might be better suited for identifying people who could benefit from hearing aids than an objective screen instrument (e.g. screen audiometer). However, the only randomised controlled trial to the effectiveness of screening so far showed that screening with an objective screen instrument led to a larger increase in the 1-year incidence of hearing aid use than screening with a subjective screen instrument (Yueh et al., 2010). Screening with an objective screen instrument was also found to be more cost-effective than screening with a subjective screen instrument or an objective and subjective instrument combined (Liu et al., 2011; Morris et al., 2013). Besides, Dutch GPs are more likely to refer someone with objectively assessed hearing impairment to a hearing care professional than someone with subjective hearing complaints alone (Eekhof et al., 2000). We therefore recommend using an objective screen instrument for nationwide adult hearing screening. This thesis addressed the harms, effects, costs, and costeffectiveness of screening with an objective screen instrument only. The conclusions may not apply to screening with a subjective screen instrument.

\section{Rehabilitation options other than hearing aids}

Hearing aids are not acceptable to everyone (Chapter 2; Chapter 4; Davis et al., 2007), nor everyone who tries hearing aids is able to benefit (Chapter 3; Chapter 4). Several alternatives are available, like assistive listening devices and communication training programmes targeting speech recognition improvement or communication management through speech reading training, hearing tactics, and coping training. People may not always be informed about these alternatives. For instance, none of the interview 
participants were aware of the existence of rehabilitation options other than hearing aids. This may be the result of how hearing care is organised in the Netherlands. Adults with hearing complaints can turn directly to the hearing aid dispenser nowadays. Intervention of the general practitioner, audiologist, or Ear Nose Throat specialist is not necessary anymore. As a consequence, the task of providing information on rehabilitation options mainly lies at the hearing aid dispenser. It is therefore important that a screening programme includes provision of information on all possible rehabilitation options and on where or whom to turn to for each type of rehabilitation.

Adults who seek help because they screened positive for hearing impairment probably have fewer and milder hearing complaints than (non-screened) self-motivated helpseekers. Hence, their perceived need for rehabilitation and their willingness to try hearing aids will be lower and, if they would purchase hearing aids, the risk that they become non-users will be higher (Chapter 3). People with mild hearing impairment are more likely to choose communication training than hearing aid fitting when offered both options (Laplante-Lévesque et al., 2012a). Therefore, communication training may be of great value in a screening setting. Unfortunately, we could not assess the value of offering communication training after screening with our Markov model because of unavailability of data on communication training uptake and on its effect on quality of life. Communication training is known to have a long-term effect on reducing hearing handicap (Hickson et al., 2007), but its effect on quality of life has not been examined yet.

Also in the study on the effects and costs of a screening and rehabilitation programme in residential care homes for the elderly (Chapter 4), rehabilitation was limited to hearing aid fitting. Maybe more residents would have benefitted from the programme if alternative rehabilitation options had been offered as well. However, since the main reason for elderly people for not taking up hearing aids is that they are more concerned with trying to improve other aspects of their functioning which they perceive as more problematic than their hearing impairment (Gussekloo et al., 2003), they might not be motivated to put effort in communication training either.

\section{Unidentified harms of screening}

A limitation of the qualitative study to adults' reactions on screening (Chapter 2 ) is that we cannot guarantee that all harmful effects have been captured. We interviewed adults who screened positive for hearing impairment and who reported to never or only occasionally have hearing complaints because we expected that, if screening would have a harmful effect, this would most likely become apparent in adults who unexpectedly screened positive. Whether screening can harm other screen participants was not studied. However, people whose screen outcome agrees with their perceived hearing status are unlikely to be harmed by the screening and people who unexpectedly screen negative will probably just be glad to hear that their hearing is not so bad after all. 
Another reason for why we cannot guarantee that all harms have been identified is that only seven screen participants were interviewed. Although there were indications that data saturation had been reached, interviews with more adults would have been needed for confirmation. We were unable to interview more persons due to the difficulty to recruit participants and time constraints. Despite the efforts of several recruiters, it took 20 months to recruit the seven participants. There are several possible explanations for this difficult recruitment. First, the number of eligible people might have been low because few people without hearing complaints have their hearing tested in the absence of a systematic screening programme. Secondly, eligible people might not have been willing to participate because the test outcome may have been painful for them. Thirdly, eligible people might have refused to participate because they felt indignant about being unfairly labelled as hearing-impaired. And finally, eligible people might have been reluctant to participate because they feared the researcher would try to persuade them to purchase hearing aids. We regret that we did not register the number of eligible adults, the number of eligible adults refusing participation, and the reason(s) for refusal.

\section{Bias in target identification by noise exposure}

A limitation of the observational study to potential targets for high-risk group screening (Chapter 5) was that information on noise exposure was not available. Crosssectional studies have consistently reported an association between noise exposure and hearing loss (Van Eyken et al., 2007). The proportion of explained interindividual variance in baseline hearing thresholds would probably have been higher if information on noise exposure had been included. Furthermore, the effect size of gender and type of occupation might have been slightly lower in a model that includes noise exposure because men and people with manual occupation are generally more likely to be exposed to noise than women and people with intellectual occupation. We do not expect that the gender difference would disappear completely after correction for noise exposure since previous studies to predictors of hearing loss in which noise exposure was corrected for found a gender difference as well. The effects of educational level, cardiovascular disease, diabetes, systemic inflammatory disease, hypertension, obesity, waist circumference, smoking, and physical activity level would probably not be influenced by inclusion of noise exposure in the model.

\section{Measurement of quality of life gain}

The utility estimates that we used in the state transition model to determine gain in quality-adjusted life years were based on the Health Utility Mark Index 3 (HUI3) questionnaire because this instrument is the most sensitive to changes in utility scores after hearing aid fitting. Other multi-attribute utility instruments, like the HUI2, EQ-5D, and SF-6D, are less sensitive to such changes or even unable to measure a change at all (Barton et al., 2004; Bess, 2000; Grutters et al., 2007; Joore et al., 2003b; Vuorialho et al., 2006). The HUI3 is the recommended instrument for measuring utility values to assess health-related quality of life in hearing impairment because its good validity and 
responsiveness (Yang et al., 2013). If another multi-attribute utility instrument had been used, adult hearing screening may not have been cost-effective.

\section{Current practice in hearing care}

In the state transition model, we used data on current practice from up to 15 years ago because more recent data was unavailable. Since several changes took place in hearing care in the past 15 years, the current practice scenario in the state transition model may not adequately represent actual current practice. Hearing aid technology has been greatly improved, for example, which may have led to higher hearing aid uptake after a trial, lower hearing aid discard, or greater benefit. Another important change is that hearing aid dispensing companies have started offering free screening and advertise about this extra service. Also, telephone and internet screening tests have become freely available to the population. If these existing screenings had been taken into account in the current practice scenario, the incremental effect of the modelled screening strategies compared to current practice might have been lower. However, if the incremental effect of the screening strategies would be only half as high as modelled, the incremental cost-effectiveness ratio would remain below $€ 20000$ for all nondominated screening strategies, indicating that nationwide implementation of a (noncommercial) screening programme would still be cost-effective.

\section{Conclusions and recommendations}

We aimed to gain more insight into how to design an adult hearing screening programme for nationwide implementation in the Netherlands by answering the following four research questions: (1) Does screening cause harm and how can potential harms be prevented or minimised? (2) What should the screening programme include to prevent non-use of dispensed hearing aids? (3) What is the best target population? (4) What is the best screen instrument?

We conclude that, to minimise the harms and maximise the effectiveness of adult hearing screening, effort should be put in convincing screen participants that the test is valid. One may deal with screen participants' concerns on the test validity by giving a clear explanation of how the test works, by offering the screening in a silent environment without distracting factors, or by offering immediate rescreening. It is also important that the screening programme includes provision of information on all possible rehabilitation options and on where and whom to turn to for each type of rehabilitation. The likelihood that adults who purchase hearing aids become non-users will probably decrease when hearing aid fitting is chosen after careful consideration of all alternatives, including doing nothing. With regard to the selection of the target population, we found that the screening programme should be targeted at the general population aged 50 , with rescreening at ages $55,60,65$, and 70 . One may consider offering screening to women at a later age than to men. People living in care homes for the elderly form no suitable target for screening. Although the majority of the 
residents are hearing impaired and do not own hearing aids, very few residents purchase hearing aids during the screening and rehabilitation programme. Based on the state transition model, it can be concluded that adult hearing screening is costeffective compared to no screening. Inviting people by letter to do the internet test was the most cost-effective screening strategy, followed by inviting people to do the telephone test. Opportunistic screening at the GP practice using a hand-held screening device or a standard audiometer was generally more costly and less effective.

Since internet screening at age 50 , repeated at ages $55,60,65$, and 70 was found to be cost-effective, generally harmless, and highly accessible, we consider it eligible for nationwide implementation. However, before implementing this screening programme nationwide, we recommend a geographically localised pilot study of 1 year with internet screening at age 50 to verify whether the assumptions made in the costeffectiveness model for the first year hold up. A control group should be included to gain insight in the additional value of the screening programme next to existing screening programmes. Outcome measures that should be evaluated at the end of the pilot include: screen participation; harms of screening; steps taken in help-seeking in relation to the screen outcome; rehabilitation decisions; hearing aid use by people who purchase hearing aids; and the effects of rehabilitation on hearing disability, hearing handicap, and quality of life. Gender differences in these outcome measures should be examined to inform the decision on whether or not to start offering screening to women at a later age than to men, which seemed a promising strategy to increase the cost-effectiveness of screening based on the gender difference in the risk for hearing impairment.

Our findings suggest that offering hearing aid self-efficacy training may be a fruitful strategy to prevent non-use of dispensed hearing aids. We therefore recommend development and validation of a Dutch questionnaire to measure hearing aid selfefficacy, development of a hearing aid self-efficacy training programme, and research on the (cost-)effectiveness of such a training programme. Another area for future research is the value of the ear age in adult hearing screening. Presenting the screen outcome as an ear age seemed to be a useful strategy for adults under age 65. A longitudinal quantitative study is needed to assess whether a screen outcome presented as an ear age is indeed more effective in stimulating middle-aged adults to seek help and start rehabilitation than a screen outcome presented differently. In addition, more research is needed to the effects of assistive listening devices and communication training programmes on the quality of life of adults with a hearing impairment and to the cost-effectiveness of these rehabilitation options.

Care home organisations willing to invest in improving the communication and social engagement of the residents are recommended to consider environmental changes, like utilization of sound absorbent materials in common rooms or changes in seating arrangements (Hickson, 2009; Looi et al., 2004; Pryce \& Gooberman-Hill, 2012; Tolson \& McIntosh, 1997). While a screening and rehabilitation programme targeted at the individual is beneficial for only a minority of the present residents, environmental improvements might be beneficial for all present residents as well as for all future 
residents (Hickson, 2009). Furthermore, since hearing aid owners living in care homes for the elderly were highly interested in consulting a hearing care professional, we recommend future research to strategies to address this apparent unmet need for aftercare. 


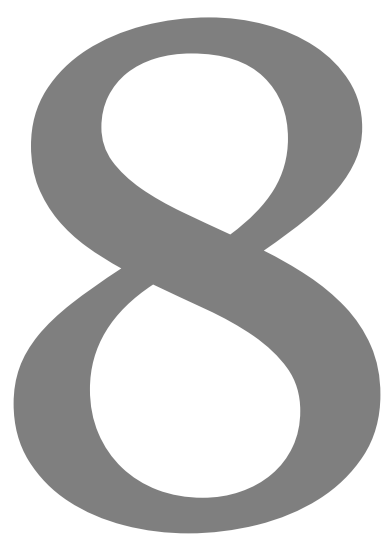

Valorisation 



\section{A national hearing screening programme for the general adult population}

Hearing loss is an important public health problem because of the large number of people involved and the negative consequences for affected, untreated people and their significant others (Chapter 1). Adult hearing screening has been proposed as a potential strategy to motivate adults to seek help early (Davis et al., 2007; Thodi et al., 2013; Yueh et al., 2003). In 2003, on the authority of the Dutch minister of Health, Welfare and Sports, the Advisory Council on Health Research reviewed the literature on adult hearing screening and concluded that before implementing a national adult hearing screening programme, more information is needed on its cost benefit ratio and on organisational strategies to optimise this ratio (Raad voor Gezondheidsonderzoek, 2003). More specific, the council recommended research to the costeffectiveness of screening, the right time for screening, the best target population, the acceptability of screening, the willingness to participate, strategies to improve participation, and factors that limit the effectiveness. Since this thesis addresses several of these topics, it contains valuable information for the Dutch government.

We interviewed recently screened adults about their reactions to the hearing screening test (Chapter 2). The participants stated to have no difficulties with the acceptability of the screening. This was to be expected because the test was noninvasive and because they had done the test by their own free will. The screening did not cause severe harms although some participants expressed feelings of indignation because they felt unfairly labelled as hearing-impaired. They believed that the test validity was poor. Adults with a positive judgment on the test validity were more likely to accept the screen outcome. Thus, to minimise the harms and maximise the effectiveness of screening, effort should be put in convincing screen participants that the test is valid. Our findings suggest that one may deal with screen participants' concerns on the test validity by giving a clear explanation of how the test works, by offering the screening in a silent environment without distracting factors, and by offering immediate rescreening (using either the same or another screening test). It will be wise to explore potential doubts people may have about the validity of a particular screening test and to include measures that may remove these doubts before nationwide implementation of the screening programme. For example, people may have doubts about the validity of the internet test because they do not understand how the volume level of their computer boxes does not influence the test outcome. Such concern can be anticipated by addressing the topic in the screen invitation letter or on the website before the test starts. Also hearing aid dispensers who are currently offering hearing screening may be interested in Chapter 2 because knowing what reactions to screening are to be expected and why, gives them the opportunity to anticipate.

To identify potential targets for high-risk group screening, we performed an observational study to predictors of hearing acuity (Chapter 5). Predictors included in this study were age, gender, type of occupation, educational level, cardiovascular disease, diabetes, systemic inflammatory disease, hypertension, obesity, waist circumference, smoking, and physical activity level. Cross-sectional as well as longitudinal regression analyses were performed. In the age-stratified regression 
models, only $11 \%$ to $21 \%$ of the interindividual variance in hearing thresholds was explained by the non-audiometric predictors, which suggests that interindividual differences in hearing thresholds are primarily attributed to genetic variation. The predictors that made a statistically significant contribution to the explanation of the interindividual variance in baseline and future hearing thresholds were higher age, male gender, manual occupation, and large waist circumference. The effects of the last two predictors were very small which makes them unsuitable as a base for target selection. The mean hearing thresholds of middle-aged and older men were found to be comparable to those of women who were approximately 8 to 9 years older, therefore, offering screening to women at a later age than to men may be considered in order to improve the cost-effectiveness of screening.

The observational study (Chapter 5 ) gave insight in the relationship between age and hearing thresholds but did not directly provide information on the right time for screening. To determine the right time for screening, we examined the effect of target age on the cost-effectiveness ratio of screening using a state transition model (Chapter 6). The effect of offering screening later to women than to men could unfortunately not be assessed with this model because gender-specific data on help-seeking and hearing aid uptake was unavailable. The model was used to calculate the costs per quality-adjusted life year (QALY) of current practice (no screening) and 76 adult hearing screening strategies. The screening strategies varied in the age at first screening (either $50,55,60,65$, or 70 years), the number of repeated screenings (up to five repetitions), the time interval between repeated screenings (either 5 or 10 years), and the type of screening which was either telephone screening, internet screening, screening at the practice of the general practitioner (GP) with a hand-held screening device, or screening at the GP practice with a standard audiometer. The two screenings at the GP practice were opportunistic strategies since the screenings were offered to people at the moment they visited the GP practice for whatever reason. For the telephone and internet screening, on the other hand, all adults from a certain age were invited by letter. In the Netherlands, screening is deemed cost-effective and may be considered for nationwide implementation if the costs are below $€ 20000$ per QALY (Van den Berg et al., 2008). We found that all screening strategies were cost-effective compared to current practice. For each type of screening, the most cost-effective strategy was targeting the screening at people aged 50 with rescreening at ages 55,60 , 65 , and 70 . Opportunistic screening at the GP practice was generally more costly and less effective than telephone or internet screening. Internet screening was slightly more cost-effective than telephone screening.

Internet screening at age 50, repeated at ages 55, 60, 65, and 70 was the most costeffective strategy, costing $€ 3699$ per QALY (Chapter 6). At a threshold of $€ 20000$ per QALY this strategy had a probability of $100 \%$ to be cost-effective compared to current practice and a probability of $69 \%$ to be more cost-effective than any other strategy. The internet test used in this study was the National Hearing Test (www.hoortest.nl) that was developed by Smits and colleagues (2006). It is a non-commercial fully automatic adaptive speech-in-noise test that uses digit-triplets as speech material. The test is highly accurate (Leensen et al., 2011). Advantages of the internet test with 
regard to the test validity are that people can control the test environment (by removing distracting factors like environmental noise) and that they can redo the test as often as needed for them to believe the outcome. Other advantages of the internet test are that people do not have to leave their house for testing and that they can do the test at any time that suits them. Internet access hardly forms a barrier for internet screening nowadays in the Netherlands as $98 \%$ of adults aged between 45 and 55 years, $94 \%$ of adults aged between 55 and 65 years, and $85 \%$ of adults aged between 65 and 75 years have access to internet at their home (Statistics Netherlands, 2013).

Since internet screening at age 50, repeated at ages 55, 60, 65, and 70 was found to be cost-effective, generally harmless, and highly accessible, we consider it eligible for nationwide implementation. Mentioning the telephone version of the National Hearing Test in the invitation letter for internet screening should be considered to make screening accessible for the few adults without internet access as well. Before implementing this screening programme nationwide, we recommend a geographically localised pilot study of 1 year with internet screening at age 50 to verify whether the assumptions made in the cost-effectiveness model for the first year hold up. A control group should be included to gain insight in the additional value of the screening programme next to existing screening programmes. Outcome measures that should be evaluated at the end of the pilot include: screen participation; harms of screening; steps taken in help-seeking in relation to the screen outcome; rehabilitation decisions; hearing aid use by people who purchase hearing aids; and the effects of rehabilitation on hearing disability, hearing handicap, and quality of life. Gender differences in these outcome measures should be examined to inform the decision on whether or not to start offering screening to women at a later age than to men, which seemed a promising strategy to increase the cost-effectiveness of screening based on the gender difference in the risk for hearing impairment.

The findings in this thesis indicate that implementing a national adult hearing screening programme will be a cost-effective strategy to improve the wellbeing of the general adult population. Implementation of such a screening programme will have positive consequences for hearing care professionals as well because their clientele will probably increase, resulting in a higher turnover and higher profits.

\section{Comprehensive counselling to prevent non-use of dispensed hearing aids}

Chapter 3 gives insight in potential strategies to prevent non-use of dispensed hearing aids. Hearing aid dispensers may use our findings to optimise their services. An important strategy to prevent non-use of dispensed hearing aids is by offering comprehensive counselling and training during the hearing aid trial. Boas and colleagues (2001) found that a counselling programme was a cost-effective strategy to decrease non-use among new hearing aid owners. Our study identified two topics that should definitely be addressed in a counselling programme. First, the programme should address the limitations of hearing aids. People should be assisted to understand and accept the hearing aids' limitations. Secondly, the counselling programme should address hearing aid self-efficacy: people should become confident that they are able to 
become successful hearing aid users. Someone's hearing aid self-efficacy can be measured at the time of hearing aid fitting, using the MARS-HA (measure of audiologic rehabilitation self-efficacy for hearing aids) questionnaire developed by West and Smith (2007). Knowing someone's level of hearing aid self-efficacy can assist the hearing care professional in determining whether or not the person needs intensive hearing aid self-efficacy training during the hearing aid trial in order to become a successful hearing aid user. By building hearing aid self-efficacy, adults will be more motivated to adjust to hearing aids, persevere when hearing aid difficulties arise, and apply more effort toward successful hearing aid use. Smith and West (2006) and Meyer and colleagues (2014) described a number of strategies for enhancing hearing aid self-efficacy, including role play and motivational coaching by hearing care professionals or significant others.

\section{Market opportunities related to hearing care for elderly care home residents}

Most residents of care homes for the elderly have a hearing loss, but only a minority of them owns hearing aids (Cohen-Mansfield \& Taylor, 2004; Stumer at al., 1996; Tolson, 1997; Tsuruoka et al., 2001). A possibly lucrative business strategy for hearing aid dispensers to reach this large pool of potential clients is by offering in-house hearing screening and rehabilitation. The evaluation study that is reported on in Chapter 4 may be of interest to hearing aid dispensers because it gives insight in the size of the potential hearing aid client pool in residential care homes for the elderly in the Netherlands and because it shows the effect of offering an in-house hearing screening and rehabilitation programme on hearing aid sales. Assuming that screen participation among residents without previously fit hearing aids was independent of their audiometric hearing status, it could be calculated that $92 \%$ of the residents of the included care homes had a hearing loss of $35 \mathrm{~dB}$ or more (best-ear pure-tone average at 1,2 , and $4 \mathrm{kHz}$ ). Approximately $72 \%$ of the residents with a hearing loss, that is $66 \%$ of all residents, had no hearing aid(s) at the start of the programme. The study was performed in $\mathbf{2 0 1 0}$ when a binaural hearing loss was required for (partial) hearing aid reimbursement. Since January 2013 also people with a monaural hearing loss are entitled to partial hearing aid reimbursement. Consequently, the pool of potential new hearing aid clients in care homes for the elderly might be even greater than $66 \%$ nowadays. The willingness to try hearing aids among the hearing impaired residents without a previously fit hearing aid was low (14\%), as was the success rate of the hearing aid trials (46\%). Compared to the large number of potential new hearing aid clients (66\% of all residents), the actual number of new hearing aid owners at the end of the programme was disappointing ( $4 \%$ of all residents). This finding is important for hearing aid dispensers who consider offering hearing screening and rehabilitation in residential care homes for the elderly.

We also found that approximately one-fifth of the care home residents who already owned hearing aids were interested in consulting a hearing care professional and that two-thirds of them had their hearing aids adjusted or replaced during the programme. This reflects a high level of unmet need for aftercare. Hearing aid dispensers may 
therefore consider to regularly visit care homes to offer hearing aid aftercare. However, taking into account the absolute number of hearing aid owners per care home, offering in-house aftercare might not be very profitable for hearing aid dispensers. Each care home housed on average 88 adults: 65 without and 23 with hearing aids. Four or five of the hearing aid owning residents were interested in consulting a hearing care professional and three of them chose for hearing aid adjustment or replacement. This finding informs hearing aid dispensers on the expected value of offering aftercare in care homes for the elderly.

The screening and rehabilitation programme that we organised in the care homes resulted in only a slight increase in hearing aid ownership among the residents with a hearing loss (from $28 \%$ to $33 \%$ ). The low hearing aid uptake during the programme indicates that most of the oldest elderly without hearing aids do not choose for hearing rehabilitation by hearing aid fitting, not even when possible mobility barriers are eliminated. Gussekloo and colleagues (2003), who came to a similar conclusion, reported that the major reason for not starting rehabilitation was that most elderly were at the same time concerned with trying to improve other aspects of their functioning which they perceived as more problematic than their hearing loss. This is probably also the reason for the low programme effectiveness in our study since care homes typically house frail elderly suffering from multiple comorbidities (ActiZ, 2010). We expect that the programme's effectiveness would not have been much higher if communication training had been offered as an alternative to hearing aid fitting because learning new communication tactics also costs effort. Therefore, care home organisations willing to invest in improving the communication and social engagement of the residents are recommended to consider environmental changes, like utilization of sound absorbent materials in common rooms or changes in seating arrangements (Hickson, 2009; Looi et al., 2004; Pryce \& Gooberman-Hill, 2012; Tolson \& Mclntosh, 1997). While a screening and rehabilitation programme targeted at the individual is beneficial for only a minority of the present residents, environmental improvements might be beneficial for all present residents as well as for all future residents (Hickson, 2009). A market opportunity for hearing care professionals is offering consultancy services to care homes for the elderly regarding optimisation of the listening environment in the (common) rooms. 

Bibliography 

ActiZ. Overdrachtsdossier van zorgondernemers 2010: Verpleeg- en verzorgingshuiszorg, Thuiszorg, Kraamen Jeugdgezondheidszorg. Utrecht, The Netherlands: author, 2010.

Adams-Wendling L, Pimple C, Adams S, \& Titler MG. Nursing management of hearing impairment in nursing facility residents. J Gerontol Nurs 2008;34:9-17.

Agrawal Y, Platz EA, \& Niparko JK. Prevalence of hearing loss and differences by demographic characteristics among US adults: data from the National Health and Nutrition Examination Survey, 1999-2004. Arch Intern Med 2008;168:1522-1530.

Akbayir N, Caliş AB, Alkim C, Sökmen HM, Erdem L, Ozbal A, Bölükbaş F, \& Akbayir N. Sensorineural hearing loss in patients with inflammatory bowel disease: a subclinical extraintestinal manifestation. Dig Dis Sci 2005;50:1938-1945.

Andermann A, Blancquaert I, Beauchamp S, \& Déry V. Revisiting Wilson and Jungner in the genomic age: a review of screening criteria over the past 40 years. Bull World Health Organ 2008;86:317-319.

Arlinger S. Negative consequences of uncorrected hearing loss: a review. Int J Audiol 2003;42:17-20.

AZOS working group. Final report AZOS [in Dutch]. Diemen, The Netherlands: Health Care Insurance Board, 2006.

Bagai A, Thavendiranathan P, \& Detsky AS. Does this patient have hearing impairment? JAMA 2006;295:416428.

Baguley D. Evaluation of early screening for hearing disability against NSC Handbook Criteria. London, United Kingdom: UK National Screening Committee, 2008. Available at: www.screening.nhs.uk/hearing-adult.

Barton GR, Bankart J, Davis AC, \& Summerfield QA. Comparing utility scores before and after hearing-aid provision: results according to the EQ-5D, HUI3 and SF-6D. Appl Health Econ Health Policy 2004;3:103105.

Bertoli S, Staehelin K, Zemp E, Schindler C, Bodmer D, \& Probst R. Survey on hearing aid use and satisfaction in Switzerland and their determinants. Int J Audiol 2009;48:183-195.

Bess $\mathrm{FH}$. The role of generic health-related quality of life measures in establishing audiological rehabilitation outcomes. Ear Hear 2000;21:S74-79.

Boas $G$, van der Stel H, Peters H, Joore $M$, \& Anteunis L. Dynamic modeling in medical technology assessment: fitting hearing aids in The Netherlands. Int J Technol Assess Health Care 2001;17:618-625.

Boer\&Croon. Abolition of the reimbursement limits for hearing aids [in Dutch]. Amsterdam, The Netherlands: author, 2010.

Brant LJ, Gordon-Salant S, Pearson JD, Klein LL, Morrell CH, Metter EJ, \& Fozard JL. Risk factors related to age-associated hearing loss in the speech frequencies. J Am Acad Audiol 1996;7:152-160.

Braun V, \& Clarke V. Using thematic analysis in psychology. Qual Res Psychol 2006;3:77-101.

Brink $\mathrm{P}$, \& Stones $\mathrm{M}$. Examination of the relationship among hearing impairment, linguistic communication, mood, and social engagement of residents in complex continuing-care facilities. Gerontologist 2007;47:633-641.

Chao TK, \& Chen TH. Cost-effectiveness of hearing aids in the hearing-impaired elderly: a probabilistic approach. Otol Neurotol 2008;29:776-783.

Chia EM, Wang JJ, Rochtchina E, Cumming RR, Newall P, \& Mitchell P. Hearing impairment and healthrelated quality of life: the Blue Mountains Hearing Study. Ear Hear 2007;28:187-195.

Chisolm TH, Johnson CE, Danhauer JL, Portz LJ, Abrams HB, Lesner S, McCarthy PA, \& Newman CW. A systematic review of health-related quality of life and hearing aids: final report of the American Academy of Audiology Task Force On the Health-Related Quality of Life Benefits of Amplification in Adults. J Am Acad Audiol 2007;18:151-183.

Chou R, Dana T, Bougatsos C, Fleming C, \& Beil T. Screening adults aged 50 years or older for hearing loss: a review of the evidence for the U.S. Preventive Services Task Force. Ann Intern Med 2011;154:347-355.

Cohen-Mansfield J, \& Taylor JW. Hearing aid use in nursing homes. Part 1: prevalence rates of hearing impairment and hearing aid use. J Am Dir Assoc 2004;5:283-288.

Cox RM, Alexander GC, \& Gray GA. Who wants a hearing aid? Personality profiles of hearing aid seekers. Ear Hear 2005;26:12-26.

Cruickshanks KJ, Tweed TS, Wiley TL, Klein BE, Klein R, Chappell R, Nondahl DM, \& Dalton DS. The 5-year incidence and progression of hearing loss: the Epidemiology of Hearing Loss Study. Arch Otolaryngol Head Neck Surg 2003;129:1041-1046.

Davis AC, Ostri B, \& Parving A. Longitudinal study of hearing. Acta Otolaryngol Suppl 1991;476:12-22.

Davis A, Stephens D, Rayment A, \& Thomas K. Hearing impairments in middle age: the Acceptability, Benefit and Cost of Detection (ABCD). Br J Audiol 1992;26:1-14. 
Davis A, Smith P, Ferguson M, Stephens D, \& Gianopoulos I. Acceptability, benefit and costs of early screening for hearing disability: a study of potential screening tests and models. Health Technol Assess 2007;11:1-294.

DBC-Onderhoud. User document Part 2: Explanation tariff changes, Version 1.0. Appendix 4.4: duration of audiological care activities [in Dutch]. Utrecht: author, 2011.

Dreschler WA, Wikkerink JA, van Troost M, \& Boymans M. Compensation of hearing impairments: technical possibilities of hearing aids and indications [in Dutch]. Amsterdam: Stichting Platform for Audiological Clinical Testing, 2009.

Drummond MF, Sculpher MJ, Torrance GW, O’Brien BJ, \& Stoddart GL. Methods for the economic evaluation of health care programmes ( $3^{\text {rd }}$ edition). New York: Oxford University Press, 2005.

Duijvestijn JA, Anteunis LJC, Hoek CJ, van den Brink RHS, Chenault MN, \& Manni JJ. Help-seeking behaviour of hearing-impaired persons aged $\geq 55$ years: effect of complaints, significant others and hearing aid image. Acta Otolaryngol 2003;123:846-850.

Duijvestijn JA, Grutters JP, Chenault MN, Joore MA, Manni JJ, \& Anteunis L. Shared care for hearing complaints: guideline effects on patient flow. J Eval Clin Pract 2011;17:209-214.

Eekhof JA, de Bock GH, Schaapveld K, \& Springer MP. Screening for hearing and visual loss among elderly with questionnaires and tests: which method is the most convincing for action? Scand J Prim Health Care 2000;18:203-207.

Engelund G. "Time for hearing" - Recognising process for the individual: a grounded theory. Unpublished doctoral dissertation, University of Copenhagen, Denmark, 2006.

Eurostat. News Release 199/2013: internet access and use in 2013. Luxembourg: Eurostat Press Office, 2013.

Fenwick E, Claxton K, \& Sculpher M. Representing uncertainty: the role of cost-effectiveness acceptability curves. Health Econ 2001;10:779-787.

Foss KA. (De)stigmatizing the silent epidemic: representations of hearing loss in entertainment television. Health Commun 2014;29:888-900.

Fransen E, Topsakal V, Hendrickx JJ, van Laer L, Huyghe JR, van Eyken E, Lemkens N, Hannula S, Mäki-Torkko E, Jensen M, Demeester K, Tropitzsch A, Bonaconsa A, Mazzoli M, Espeso A, Verbruggen K, Huyghe J, Huygen PL, Kunst S, Manninen M, Diaz-Lacava A, Steffens M, Wienker TF, Pyykkö I, Cremers CW, Kremer H, Dhooge I, Stephens D, Orzan E, Pfister M, Bille M, Parving A, Sorri M, van de Heyning P, \& van Camp G. Occupational noise, smoking, and a high body mass index are risk factors for age-related hearing impairment and moderate alcohol consumption is protective: a European population-based multicenter study. J Assoc Res Otolaryngol 2008;9:264-276.

Garstecki DC, \& Erler SF. Hearing loss, control, and demographic factors influencing hearing aid use among older adults. J Speech Lang Hear Res 1998;41:527-537.

Gates GA, \& Cooper JC. Incidence of hearing decline in the elderly. Acta Otolaryngol 1991;111:240-248.

Gopinath B, Wang JJ, Schneider J, Burlutsky G, Snowdon J, McMahon CM, Leeder SR, \& Mitchell P. Depressive symptoms in older adults with hearing impairments: the Blue Mountains Study. J Am Geriatr Soc 2009;57:1306-1308.

Green KA, McGwin G Jr, \& Owsley C. Associations between visual, hearing, and dual sensory impairments and history of motor vehicle collision involvement of older drivers. J Am Geriatr Soc 2013;61:252-257.

Groenland R. Beter Horen gets to know its clients better! [in Dutch]. Unpublished thesis, Hogeschool van Amsterdam, The Netherlands, 2011.

Grutters JP, Joore MA, van der Horst F, Verschuure H, Dreschler WA, \& Anteunis L. Choosing between measures: comparison of EQ-5D, HUI2 and HUI3 in persons with hearing complaints. Qual Life Res 2007;16:1439-1449.

Grutters JP, Joore MA, van der Horst F, Stokroos RJ, \& Anteunis L. Decision-analytic modeling to assist decision making in organizational innovation: the case of shared care in hearing aid provision. Health Serv Res 2008;43:1662-1673.

Gussekloo J, de Bont LE, von Faber M, Eekhof JA, de Laat JA, Hulshof JH, van Dongen E, \& Westendorp RG. Auditory rehabilitation of older people from the general population: the Leiden 85 -plus study. $\mathrm{Br} J \mathrm{Gen}$ Pract 2003;53:536-540.

Hakkaart-van Roijen L, Tan SS, \& Bouwmans CAM. Manual for costing research [in Dutch]. Diemen, The Netherlands: College voor Zorgverzekeringen, 2010.

Hartley D, Rochtchina E, Newall P, Golding M, \& Mitchell P. Use of hearing aids and assistive listening devices in an older Australian population. J Am Acad Audiol 2010;21:642-653. 
Health Care Insurance Board. GIP database, 2011. Available from: www.gipdatabank.nl. [Accessed April 11, 2014]

Hickson L, Worrall L, \& Scarinci N. A randomized controlled trial evaluating the Active Communication Education program for older people with hearing impairment. Ear Hear 2007;28;212-230.

Hickson L. The challenge of older people living in aged care environments. In: Proceedings of the second International Adult Conference, Hearing care for adults: the challenge of aging (Chapter 11, pp. 119122). Staefa, Switzerland: Phonak AG, 2009.

Hindhede AL. Negotiating hearing disability and hearing disabled identities. Health (London) 2012;16:169185.

Hingstman L, \& Kenens RJ. Figures from the registration of general practitioners [in Dutch]. Utrecht: NIVEL, 2011.

Hughson W, \& Westlake HD. Manual for program outline for rehabilitation of aural casualties both military and civilian. Trans Am Acad Ophthalmol Otolaryngol 1944;48:1-15.

Humes LE. The contributions of audibility and cognitive factors to the benefit provided by amplified speech to older adults. J Am Acad Audiol 2007;18:590-603.

Hwang JH, Wu CC, Hsu CJ, Liu TC, \& Yang WS. Association of central obesity with the severity and audiometric configurations of age-related hearing impairment. Obesity (Silver Spring) 2009;17:17961801.

Janacsek K, Fiser F, \& Nemeth D. The best time to acquire new skills: age-related differences in implicit sequence learning across the human lifespan. Dev Sci 2012;15:496-505.

Jolles J, Houx PJ, van Boxtel MPJ, \& Ponds RWHM, eds. The Maastricht Aging Study: determinants of cognitive aging. Maastricht, The Netherlands: Neuropsych Publishers, 1995.

Joore MA, van der Stel H, Peters HJ, Boas GM, \& Anteunis L. The cost-effectiveness of hearing-aid fitting in the Netherlands. Arch Otolaryngol Head Neck Surg 2003a;129:297-304.

Joore MA, Brunenberg DE, Chenault MN, \& Anteunis LJ. Societal effects of hearing aid fitting among the moderately hearing impaired. Int J Audiol 2003b;42:152-160.

Jupiter T, \& Spivey V. Perception of hearing loss and hearing handicap on hearing aid use by nursing home residents. Geriatr Nurs 1997;18:201-207.

Karlsmose B, Lauritzen T, Engberg M, \& Parving A. A five-year longitudinal study of hearing in a Danish rural population aged 31-50 years. Br J Audiol 2000;34:47-55.

Kiely KM, Gopinath B, Mitchell P, Browning CJ, \& Anstey KJ. Evaluating a dichotomized measure of selfreported hearing loss against gold standard audiometry: prevalence estimates and age bias in a pooled national data set. J Aging Health 2012a;24:439-458.

Kiely KM, Gopinath B, Mitchell P, Luszcz M, \& Anstey KJ. Cognitive, health, and sociodemographic predictors of longitudinal decline in hearing acuity among older adults. J Gerontol A Biol Sci Med Sci 2012b;67:9971003.

Kim LG, Thompson SG, Briggs AH, Buxton MJ, \& Campbell HE. How cost-effective is screening for abdominal aortic aneurysms? J Med Screen 2007;14:46-52.

Knudsen LV, Öberg M, Nielsen C, Naylor G, \& Kramer SE. Factors influencing help seeking, hearing aid uptake, hearing aid use and satisfaction with hearing aids: a review of the literature. Trends Amplif 2010;14:127-154.

Knudsen LV, Laplante-Lévesque A, Jones L, Preminger JE, Nielsen C, Lunner T, Hickson L, Naylor G, \& Kramer SE. Conducting qualitative research in audiology: a tutorial. Int J Audiol 2012;51:83-92.

Kochkin S. MarkeTrak VIII: consumer satisfaction with hearing aids is slowly increasing. Hear J 2010;63:19-27.

Koopman J, Davey E, Thomas N, Wittkop T, \& Verschuure H. How should hearing screening tests be offered? Int J Audiol 2008;47:230-237.

Kumar M, Hickey S, \& Shaw S. Manual dexterity and successful hearing aid use. J Laryngol Otol 2000;114:593-597.

Landelijke Huisartsen Vereniging. Collective Labour Agreement General Practitioners 2011 [in Dutch]. Utrecht: author, 2011.

Laplante-Lévesque A, Hickson L, \& Worrall L. What makes adults with hearing impairment take up hearing aids or communication programs and achieve successful outcomes? Ear Hear 2012a;33:79-93.

Laplante-Lévesque A, Knudsen LV, Preminger JE, Jones L, Nielsen C, Öberg M, Lunner T, Hickson L, Naylor G, \& Kramer SE. Hearing help-seeking and rehabilitation: perspectives of adults with hearing impairment. Int J Audiol 2012b;51:93-102. 
Laplante-Lévesque A, Jensen LD, Dawes $P$, \& Nielsen C. Optimal hearing aid use: focus groups with hearing aid clients and audiologists. Ear Hear 2013;34:193-202.

Lee FS, Matthews LJ, Dubno JR, \& Mills JH. Longitudinal study of pure-tone thresholds in older persons. Ear Hear 2005;26:1-11.

Leegwater E, \& Lammerts van Bueren W. Hearing in the Netherlands [in Dutch]. Amsterdam, The Netherlands: TNS-NIPO report, 2005.

Leensen MC, de Laat JA, \& Dreschler WA. Speech-in-noise screening tests by internet, Part 1: test evaluation for noise-induced hearing loss identification. Int J Audiol 2011;50:823-834.

Lin FR, Thorpe R, Gordon-Salant S, \& Ferrucci L. Hearing loss prevalence and risk factors among older adults in the United States. J Gerontol A Biol Sci Med Sci 2011;66:582-590.

Liu CF, Collins MP, Souza PE, \& Yueh B. Long-term cost-effectiveness of screening strategies for hearing loss. J Rehabil Res Dev 2011;48:235-243.

Lockey K, Jennings MB, \& Shaw L. Exploring hearing aid use in older women through narratives. Int J Audiol 2010;49:542-549.

Looi V, Hickson L, Price A, Lee G, Mokoka A, Worrall L, Wilson J, \& Tilse C. Audiological rehabilitation in a residential aged care facility. Aust N Z J Audiol 2004;26:12-29.

Lunner T. Cognitive function in relation to hearing aid use. Int J Audiol 2003;42:S49-58.

Mahoney DF. Hearing loss among nursing home residents: perceptions and realities. Clin Nurs Res 1992;1:317-332.

Maeda K, Shimbo T, \& Fukui T. Cost-effectiveness of a community-based screening programme for chronic atrial fibrillation in Japan. J Med Screen 2004;11:97-102.

Martin FN, Krall L, \& O’Neal J. The diagnosis of acquired hearing loss: patient reactions. J Speech Lang Hear Res 1989;31:47-50.

McMahon CM, Kifley A, Rochtchina E, Newall P, \& Mitchell P. The contribution of family history to hearing loss in an older population. Ear Hear 2008;29:578-584.

Meister H, Walger M, Brehmer D, von Wedel UC, \& von Wedel H. The relationship between pre-fitting expectations and willingness to use hearing aids. Int J Audiol 2008;47:153-159.

Meyer C, \& Hickson L. What factors influence help-seeking for hearing impairment and hearing aid adaptation in older adults? Int J Audiol 2012;51:66-74.

Meyer C, Hickson L, \& Fletcher A. Identifying the barriers and facilitators to optimal hearing aid self-efficacy. Int J Audiol 2014;53:S28-37.

Michikawa T, Nishiwaki Y, Asakura K, Hillebrand G, Miyamoto K, Ono M, Kinjo Y, Akiba S, \& Takebayashi T. Sunlight exposure may be a risk factor of hearing impairment: a community-based study in Japanese older men and women. J Gerontol A Biol Sci Med Sci 2013;68:96-103.

Mitchell P, Gopinath B, Wang JJ, McMahon CM, Schneider J, Rochtchina E, \& Leeder SR. Five-year incidence and progression of hearing impairment in an older population. Ear Hear 2011;32:251-257.

Monzani D, Galeazzi GM, Genovese E, Marrara A, \& Martini A. Psychological profile and social behaviour of working adults with mild or moderate hearing loss. Acta Otorhinolaryngol Ital 2008;28:61-66.

Morris AE, Lutman ME, Cook AJ, \& Turner D. An economic evaluation of screening 60- to 70-year-old adults for hearing loss. J Public Health (Oxf) 2013;35:139-146.

Mulrow CD, \& Lichtenstein MJ. Screening for hearing impairment in the elderly: rationale and strategy. J Gen Intern Med 1991;6:249-258.

Nachtegaal J, Smit JH, Smits C, Bezemer PD, van Beek JHM, Festen JM, \& Kramer SE. The association between hearing status and psychosocial health before the age of 70 years: results from an internetbased national survey on hearing. Ear Hear 2009;30:302-312.

Nash SD, Cruickshanks KJ, Klein R, Klein BE, Nieto FJ, Huang GH, Pankow JS, \& Tweed TS. The prevalence of hearing impairment and associated risk factors: the Beaver Dam Offspring Study. Arch Otolaryngol Head Neck Surg 2011;137:432-439.

Nationaal Overleg Audiologische Hulpmiddelen. Hearing aid fitting protocol, Version 3.0: tasks and responsibilities [in Dutch]. Amersfoort, The Netherlands: author, 2009.

Nederlandse Vereniging van Audicien Bedrijven. The market for hearing aids in The Netherlands [in Dutch]. The Hague, The Netherlands: author, 2011a.

Nederlandse Vereniging van Audicien Bedrijven. Annual report 2011 [in Dutch]. Tilburg: author, $2011 \mathrm{~b}$.

Nederlandse Vereniging van Ziekenhuizen. Collective Labour Agreement Hospitals [in Dutch]. Utrecht, The Netherlands: author, 2011. 
Pearson JD, Morrell CH, Gordon-Salant S, Brant LJ, Metter EJ, Klein LL, \& Fozard JL. Gender differences in a longitudinal study of age-associated hearing loss. J Acoust Soc Am 1995;97:1196-1205.

Pronk M, Kramer SE, Davis AC, Stephens D, Smith PA, Thodi C, Anteunis LJ, Parazzini M, \& Grandori F. Interventions following hearing screening in adults: a systematic descriptive review. Int $J$ Audiol 2011;50:594-609.

Pryce $H, \&$ Gooberman-Hill R. 'There's a hell of a noise': living with a hearing loss in residential care. Age Ageing 2012;41:40-66.

Raad voor Gezondheidsonderzoek. Recommendation Hearing research, publication 43 [in Dutch]. The Hague, The Netherlands: author, 2003.

Scarinci N, Worrall L, \& Hickson L. The effect of hearing impairment in older people on the spouse. Int J Audiol 2008;47:141-151.

Schneider JM, Gopinath B, McMahon CM, Britt HC, Harrison CM, Usherwood T, Leeder SR, \& Mitchell P. Role of general practitioners in managing age-related hearing loss. Med J Aust 2010;192:20-23.

Smith SL, \& West RL. The application of self-efficacy principles in audiological rehabilitation: a tutorial. Am J Audiol 2006;15:46-56.

Smits C, Kapteyn TS, \& Houtgast T. Development and validation of an automatic speech-in-noise screening test by telephone. Int J Audiol 2004;43:15-28.

Smits C, \& Houtgast T. Results from the Dutch speech-in-noise screening test by telephone. Ear Hear 2005;26:89-95.

Smits C, Merkus P, \& Houtgast T. How we do it: the Dutch functional hearing-screening tests by telephone and internet. Clin Otolaryngol 2006;31:436-440.

Soureti A, Hurling R, Murray P, van Mechelen W, \& Cobain M. Evaluation of a cardiovascular disease risk assessment tool for the promotion of healthier lifestyles. Eur J Cardiovasc Prev Rehabil 2010;17:519-523.

Spoor A, \& Passchier-Vermeer W. Spread in hearing levels of non-noise exposed people at various ages. Int J Audiol 1969;8:328-336.

Statistics Netherlands. StatLine database. Available at: http://statline.cbs.nl/StatWeb.

Stephens SD, Callaghan DE, Hogan S, Meredith R, Rayment A, \& Davis AC. Hearing disability in people aged 50-65: effectiveness and acceptability of rehabilitative intervention. BMJ 1990;300:508-511.

Stumer J, Hickson L, \& Worrall L. Hearing impairment, disability and handicap in elderly people living in residential care and in the community. Disabil Rehabil 1996;18:76-82.

Takatsu M, Higaki M, Kinoshita H, Mizushima Y, Koizuka I. Ear involvement in patients with rheumatoid arthritis. Otol Neurotol 2005;26:755-761.

Thodi C, Parazzini M, Kramer SE, Davis A, Stenfelt S, Janssen T, Smith P, Stephens D, Pronk M, Anteunis LJ, Schirkonyer V, \& Grandori F. Adult hearing screening: follow-up and outcomes. Am J Audiol 2013;22:183-185.

Tolson D. Age-related hearing loss: a case for nursing intervention. J Adv Nurs 1997;26:1150-1157.

Tolson D, \& Mclntosh J. Listening in the care environment: chaos or clarity for the hearing-impaired elderly person. Int J Nurs Stud 1997;34:173-182.

Tsuruoka H, Masuda S, Ukai K, Sakakura Y, Harada T, \& Majima Y. Hearing impairment and quality of life for the elderly in nursing homes. Auris Nasus Larynx 2001;28:45-54.

Van Boxtel MPJ, Buntinx F, Houx PJ, Metsemakers JFM, Knottnerus A, \& Jolles J. The relation between morbidity and cognitive performance in a normal aging population. J Gerontol A Biol Sci Med Sci 1998;53:M147-M154.

Van den Berg PJ, Prins A, Verschuure H, \& Hoes AW. Effectiveness of a single and a repeated screen for hearing loss in the elderly. Audiology 1999;38:339-340.

Van den Berg M, de Wit GA, Vijgen SM, Busch MC, \& Schuit AJ. Cost-effectiveness of prevention: opportunities for public health policy in the Netherlands. Ned Tijdschr Geneeskd 2008;152:1329-1334.

Van den Brink RH, Wit HP, Kempen GI, \& van Heuvelen MJ. Attitude and help-seeking for hearing impairment. Br J Audiol 1996;30:313-324.

Van Eyken E, van Camp G, \& van Laer L. The complexity of age-related hearing impairment: contributing environmental and genetic factors. Audiol Neurotol 2007;12:345-358.

Van Thiel L. Hearing in the Netherlands [in Dutch]. Amsterdam, The Netherlands: TNS-NIPO report, 2010.

Viljanen A, Era P, Kaprio J, Pyykkö I, Koskenvuo M, \& Rantanen T. Genetic and environmental influences on hearing in older women. J Gerontol A Biol Sci Med Sci 2007;62:447-452.

Vuorialho A, Karinen $\mathrm{P}$, \& Sorri M. Effect of hearing aids on hearing disability and quality of life in the elderly. Int J Audiol 2006;45:400-405. 
Wallhagen MI, Strawbridge WJ, Shema SJ, \& Kaplan GA. Impact of self-assessed hearing loss on a spouse: a longitudinal analysis of couples. J Gerontol B Psychol Sci Soc Sci 2004;59:S190-196.

Wallhagen MI. The stigma of hearing loss. Gerontologist 2010;50:66-75.

Walma EP, Thomas S, Prins A, Grundmeyer HGLM, van der Laan JR, \& Wiersma T. Clinical practice guidelines on hypertension [in Dutch]. Huisarts Wet 2003;46:435-449.

Weinberger M. Social and psychological consequences of legitimating a hearing impairment. Soc Sci \& Med 1980;14A:213-222.

Weiner B. An attribution theory of motivation. Van Lange PAM, Kruglanski AW, \& Higgins ET, eds. Handbook of theories of social psychology (Vol 1). Thousand Oaks, CA: Sage Publications Ltd, 2012 (pp. 135-155).

West RL, \& Smith SL. Development of a hearing aid self-efficacy questionnaire. Int J Audiol 2007;46:759-771.

Wiley TL, Chappell R, Carmichael L, Nondahl DM, \& Cruickshanks KJ. Changes in hearing thresholds over 10 years in older adults. J Am Acad Audiol 2008;19:281-292.

Wilson JMG, \& Jungner G. Principles and practice of screening for disease. Genova, Switzerland: World Health Organisation, 1968.

Wingfield A, Panizzon M, Grant MD, Toomey R, Kremen WS, Franz CE, Jacobson KC, Eisen SA, \& Lyons M. A twin-study of genetic contributions to hearing acuity in late middle age. J Gerontol A Biol Sci Med Sci 2007;62:1294-1299.

World Health Organisation. Waist circumference and waist-hip ratio: report of a WHO expert consultation. Geneva, Switzerland: author, 2008.

World Health Organisation. Media centre, fact sheet $N^{\circ} 300$ : Deafness and hearing loss. Genova, Switzerland: author, 2013. Available at: http://www.who.int/mediacentre/factsheets/fs300/en/

Yang Y, Longworth L, \& Brazier J. An assessment of validity and responsiveness of generic measures of health-related quality of life in hearing impairment. Qual Life Res 2013;22:2813-2828.

Yueh B, Shapiro N, MacLean CH, \& Shekelle PG. Screening and management of adult hearing loss in primary care: scientific review. JAMA 2003;289:1976-1985.

Yueh B, Collins MP, Souza PE, Boyko EJ, Loovis CF, Heagerty PJ, Liu CF, \& Hedrick SC. Long-term effectiveness of screening for hearing loss: the Screening for Auditory Impairment - Which Hearing Assessment Test (SAI-WHAT) randomized trial. J Am Geriatr Soc 2010;58:427-434. 
Summary 

Untreated hearing loss can have multiple negative consequences including social isolation, loneliness, psychosocial distress, depression, and poor quality of life. Despite the availability of several rehabilitation options, adults generally postpone seeking help until five to ten years after the onset of their hearing loss. Adult hearing screening has been proposed as a potential strategy to motivate adults to seek help earlier. However, to be eligible for nationwide implementation, a screening programme should meet several universal criteria (Chapter 1 ).

The objective of this thesis is to gain more insight into how to design an adult hearing screening programme for nationwide implementation. The focus is on minimisation of the harms of screening, prevention of non-use of dispensed hearing aids, and selection of the target population and type of screening.

\section{Minimising harms of screening}

The most important criterion a nationwide screening programme should fulfil is that the overall benefits should outweigh the harms. To identify potential harms of screening and clues for prevention or minimisation of these harms, we performed a qualitative study on the reactions of adults without hearing complaints to being labelled as hearing-impaired based on an objective hearing screening test. This study is described in Chapter 2. The specific population was selected because we expected that, if screening would have a harmful effect, it would most likely become apparent in adults who unexpectedly screened positive for hearing impairment. Individual face-toface, semi-structured interviews were conducted with seven adults (aged 52-79 years). Thematic analysis of the interviews showed that screening causes no or little harm. The only indicator of harm in this study was that some participants felt indignant about the screen outcome because they felt they were being unfairly labelled as hearing-impaired. Expression of indignation was related to the people's beliefs with regard to the validity of the screening test. Thus, to minimise the harms of screening, effort should be put in convincing screen participants that the test is valid.

\section{Preventing non-use of dispensed hearing aids}

Another criterion a nationwide screening programme should fulfil is that there should be an acceptable treatment or rehabilitation option. Hearing aid fitting, the conventional rehabilitation option for people with hearing loss, is generally considered acceptable. Nevertheless, up to a quarter of the people who own hearing aids never use them. Chapter 3 reports on a qualitative study that aimed to increase our understanding of hearing aid non-users in order to get clues for non-use prevention. Individual face-to-face, semi-structured interviews were completed with 11 hearing aid owners (aged 54-80 years) who reported that they never or hardly ever used their hearing aids. The study findings suggest that non-use of dispensed hearing aids may partly be prevented by more careful consideration whether hearing aid fitting is indeed the most suitable rehabilitation option for a particular help-seeker before starting a hearing aid trial. Offering comprehensive counselling and hearing aid selfefficacy training during the hearing aid trial may prevent hearing aid non-use as well. 


\section{Target population}

The target population should be clearly defined before a screening programme is considered for nationwide implementation. Chapter 4 describes a pilot study on the costs and effects of a screening (pure-tone audiometry) and rehabilitation (hearing aids) programme that was organised in eight residential care homes for the elderly. The costs were $€ 1896$ per successfully rehabilitated resident. Hearing aid ownership among the residents with hearing loss increased from $28 \%$ at the start of the programme to $33 \%$ at the end. Given the limited effectiveness, we conclude that care home residents form no suitable target for a nationwide hearing screening programme. Remarkable is that approximately one-fifth of the care home residents who already owned hearing aids were interested in consulting a hearing care professional and two-thirds of them had their hearing aids adjusted or had purchased one or two new hearing aids during the programme. These numbers reflect a high level of unmet need for aftercare. Future research to strategies to address this unmet need is recommended.

Also Chapter 5 is about target selection. It describes an observational study that aimed to identify predictors of hearing thresholds (best-ear pure-tone average at 1, 2, and 4 $\mathrm{kHz}$ ) and hearing deterioration in order to define potential (high-risk) target groups for hearing screening. We analysed data from the Maastricht Aging Study, a Dutch cohort (aged 24-81 years; $\mathrm{N}=1721$ ) that was observed for 12 years. Mixed model analysis was used to calculate each participant's average hearing threshold deterioration rate during the follow-up period. We built ordinary least square linear regression models to predict the baseline threshold and deterioration rate. Potential predictors included in these models were age, gender, type of occupation, educational level, cardiovascular disease, diabetes, systemic inflammatory disease, hypertension, obesity, waist circumference, smoking, and physical activity level. Poorer baseline thresholds were found to be strongly associated with faster hearing deterioration. Higher age, male gender, manual occupation, and large waist circumference were statistically significantly associated with poorer baseline thresholds and faster deterioration. The effects of the last two predictors were very small which makes them unsuitable as a base for target selection. The mean hearing thresholds of middle-aged and older men were found to be comparable to those of women who were approximately 8 to 9 years older, therefore, offering screening to women at a later age than to men may be considered in order to improve the cost-effectiveness of screening.

The observational study described in Chapter 5 gave insight in the relationship between age and hearing thresholds but did not directly provide information on the optimal age for screening. To determine the optimal age, we examined the influence of target age on the cost-effectiveness ratio of screening using a state transition model. This model, presented in Chapter 6, was used to calculate the costs per qualityadjusted life year (QALY) of current practice (no screening) and 76 adult hearing screening strategies. The screening strategies varied in the age at first screening (either $50,55,60,65$, or 70 years), the number of repeated screenings (up to five repetitions), the time interval between repeated screenings (either 5 or 10 years), and the type of screening (four types). In the Netherlands, screening is deemed cost-effective and may 
be considered for nationwide implementation if the costs are below $€ 20000$ per QALY. We found that all screening strategies were cost-effective compared to current practice. For each type of screening, the most cost-effective strategy was targeting the screening at people aged 50 with rescreening at ages $55,60,65$, and 70.

\section{Type of screening}

The state transition model from Chapter 6 was also used to determine what type of screening would be best. We analysed and compared the costs and effects of the following four types of screening: telephone screening, internet screening, screening at the practice of the general practitioner (GP) with a hand-held screening device, and screening at the GP practice with a standard audiometer. The two screenings at the GP practice were opportunistic strategies since the screenings were offered to people at the moment they visited the GP practice for whatever reason. For the telephone and internet screening, on the other hand, all adults from a certain age were invited by letter. We found that all types of screening were cost-effective compared to current practice. Opportunistic screening at the GP practice was generally more costly and less effective than telephone or internet screening. Internet screening was slightly more cost-effective than telephone screening. Internet screening at age 50 , repeated at ages $55,60,65$, and 70 was the most cost-effective strategy, costing $€ 3699$ per QALY. At a threshold of $€ 20000$ per QALY this strategy had a probability of $100 \%$ to be costeffective compared to current practice and a probability of $69 \%$ to be more costeffective than any other strategy.

In addition, the qualitative study from Chapter 2 informed us about screen participants' thoughts on having the screen outcome presented as an ear age. The participants considered the ear age to be a valuable indicator of the severity of their hearing loss. Our findings suggest that presenting the screen outcome as an ear age would be a useful strategy, but only for adults younger than 65 years. However, a longitudinal quantitative study is needed to determine whether a screen outcome presented as an ear age is indeed more effective in stimulating middle-aged adults to seek help and start rehabilitation than a screen outcome presented differently.

\section{Discussion}

In Chapter 7 the studies presented in this thesis are reviewed and discussed. Internet screening at age 50 , repeated at ages $55,60,65$, and 70 was concluded to be costeffective, harmless, and highly accessible. We consider it eligible for nationwide implementation and recommend starting a geographically localised pilot. The screening programme should include provision of information on all possible rehabilitation options and on whom and where to turn to for each type of rehabilitation. Important areas for future research include the (cost-)effectiveness of hearing aid self-efficacy training during a hearing aid trial, the uptake and (cost-)effectiveness of rehabilitation options other than hearing aids, the impact of ear age presentation on help-seeking, and strategies to address the unmet need for hearing aid aftercare among hearing aid owners living in care homes for the elderly. 

Samenvatting 

Slechthorendheid kan veel negatieve gevolgen hebben die (deels) voorkomen of verholpen kunnen worden door communicatietraining en door het gebruik van hoortoestellen of andere hoorhulpmiddelen. Desondanks wachten volwassenen gemiddeld vijf tot tien jaar alvorens zij hulp zoeken voor hun gehoorklachten. Gehoorscreening kan mensen motiveren om eerder hulp te zoeken. Om in aanmerking te komen voor nationale implementatie moet een screeningsprogramma echter aan verschillende universele eisen voldoen (Hoofdstuk 1).

Het doel van dit proefschrift is om meer inzicht te verwerven in hoe een gehoorscreeningsprogramma voor volwassenen het best vormgegeven kan worden. De focus ligt op het beperken van schadelijke effecten van screening, preventie van niet-gebruik van verstrekte hoortoestellen en selectie van de doelpopulatie en het type screening.

\section{Schadelijke effecten van screening beperken}

Het belangrijkste criterium waaraan een nationaal screeningsprogramma moet voldoen is dat de voordelen moet opwegen tegen de nadelen. Hoofdstuk 2 beschrijft een kwalitatieve studie naar de reacties van volwassenen zonder gehoorklachten wanneer zij op basis van een objectieve screeningstest als slechthorend worden bestempeld. Het doel van deze studie was om eventuele schadelijke effecten van screening in kaart te brengen en om inzicht te krijgen in mogelijke manieren om deze schadelijke effecten te voorkomen of beperken. Deze specifieke populatie was gekozen omdat we verwachtten dat, mocht screening schadelijke effecten hebben, deze hoogstwaarschijnlijk zouden optreden bij volwassenen die onverwacht positief screenen voor slechthorendheid. Voor deze studie werden zeven volwassenen geïnterviewd binnen twee weken na de screening. De enige aanwijzing voor een schadelijk effect van screening was dat sommige deelnemers verontwaardiging uitten vanwege het feit dat zij zich onterecht bestempeld voelden als slechthorend. Verontwaardiging was vooral gerelateerd aan hun perceptie van de testvaliditeit. Om de schadelijke effecten van screening te beperken moet daarom getracht worden screendeelnemers ervan te overtuigen dat de screeningstest valide is.

\section{Preventie van niet-gebruik van verstrekte hoortoestellen}

Een ander criterium waaraan een nationaal screeningsprogramma moet voldoen is dat er een acceptabele behandeling beschikbaar dient te zijn. Hoortoestelgebruik, de gangbare behandeling voor slechthorendheid, wordt in het algemeen gezien als acceptabel. Desondanks gebruikt een kwart van de hoortoestelbezitters hun hoortoestel nooit. Hoofdstuk 3 beschrijft een kwalitatieve studie die bedoeld was om nietgebruikers beter te leren begrijpen en inzicht te verwerven in mogelijke aanknopingspunten voor preventie van niet-gebruik. We hebben 11 volwassenen geïnterviewd die hoortoestellen bezaten maar deze niet of nauwelijks gebruikten. De bevindingen suggereren dat niet-gebruik van verstrekte hoortoestellen mogelijk deels kan worden voorkomen door terughoudender te zijn met hoortoestelverstrekking. Voordat gestart wordt met een hoortoestelproef dient voor elke hulpzoeker individueel te worden 
nagegaan of hoortoestelaanpassing daadwerkelijk de meest geschikte behandeling is. Ook uitgebreide advisering en hoortoesteltraining (gericht op het vergroten van iemands zelfvertrouwen met betrekking tot succesvol hoortoestelgebruik) tijdens de hoortoestelproef draagt waarschijnlijk bij aan preventie van niet-gebruik van verstrekte hoortoestellen.

\section{Doelpopulatie}

De doelpopulatie dient duidelijk gedefinieerd te zijn wil een screeningsprogramma in aanmerking komen voor nationale implementatie. Hoofdstuk 4 presenteert een pilotstudie naar de kosten en effecten van een screening (toonaudiometrie) en rehabilitatie (hoortoestellen) programma dat georganiseerd was in acht verzorgingshuizen. De kosten bedroegen $€ 1896$ per succesvol gerehabiliteerde bewoner. Hoortoestelbezit onder slechthorende bewoners was toegenomen van $28 \%$ vóór de start van het programma tot $33 \%$ aan het einde. Aangezien slechts een beperkt aantal slechthorende bewoners in deze pilotstudie koos voor rehabilitatie concluderen we dat verzorgingshuisbewoners geen geschikte doelpopulatie vormen voor een nationaal gehoorscreeningsprogramma. Opmerkelijk is dat ongeveer een-vijfde van de bewoners die al een hoortoestel bezaten geïnteresseerd was in een consult met een hoorspecialist en dat twee-derde van hen hun hoortoestel liet aanpassen of een nieuw of extra hoortoestel aanschafte tijdens het programma. Er was blijkbaar een grote behoefte aan nazorg.

Hoofdstuk 5 rapporteert over een observationele studie naar voorspellers van gehoordrempels met als doel het verwerven van inzicht in mogelijke doelgroepen voor (hoogrisicogroep) screening. Gehoordrempels bleken niet of slechts zwak samen te hangen met het type arbeid, opleidingsniveau, cardiovasculaire aandoeningen, diabetes mellitus, chronische ontstekingsziekten, hypertensie, obesitas, tailleomvang, roken en lichamelijk activiteit. Selectie van de doelpopulatie op basis van deze factoren is daarom niet interessant. De gehoordrempels van mannen waren vergelijkbaar met die van vrouwen die 8 à 9 jaar ouder waren. Dit suggereert dat de kosteneffectiviteit van gehoorscreening mogelijk kan worden geoptimaliseerd door bij vrouwen op een latere leeftijd te beginnen met screenen dan bij mannen.

Hoewel de observationele studie van Hoofdstuk 5 inzicht gaf in de relatie tussen leeftijd een gehoordrempels konden hier geen conclusies aan verbonden worden met betrekking tot de optimale leeftijd voor screening. Om de optimale leeftijd voor screening te bepalen hebben we daarom de invloed van de leeftijd van de doelpopulatie op het kosteneffectiviteitsratio van screening onderzocht met behulp van een Markov model. Dit model, beschreven in Hoofdstuk 6, hebben we gebruikt om de kosten per voor kwaliteit gecorrigeerd levensjaar te berekenen voor de huidige praktijk (geen nationaal gehoorscreeningsprogramma) en 76 screeningsstrategieën. De strategieën varieerden in de leeftijd van de doelpopulatie bij de eerste screening $(50,55,60$, 65 of 70), het aantal herhaalde screenings (tot vijf herhalingen), de tijd tussen herhaalde screenings (vijf of tien jaar) en het type screening (vier types). In Nederland wordt screening kosteneffectief geacht als de kosten lager zijn dan $€ 20000$ per voor 
kwaliteit gecorrigeerd levensjaar. Alle screeningsstrategieën waren kosteneffectief ten opzichte van geen screening. Elk type screening was het meest kosteneffectief wanneer gestart werd op een leeftijd van 50 jaar met herhaling na 5, 10, 15 en 20 jaar.

\section{Type screening}

Het Markov model van Hoofdstuk 6 is ook gebruikt voor de selectie van het meest geschikte type screening. De volgende vier typen screening zijn ermee geanalyseerd: telefoonscreening, internetscreening, screening in de huisartspraktijk met een draagbaar screeningsapparaat en screening in de huisartspraktijk met een standaard audiometer. Voor de telefoon- en internetscreening werden mensen uitgenodigd middels een brief. De andere twee screeningstypen zijn opportunistisch: ze werden aangeboden op het moment dat iemand de huisartspraktijk bezocht, ongeacht de reden van het bezoek. Alle screeningstypen waren kosteneffectief ten opzichte van geen screening. Opportunistische screening in de huisartspraktijk was over het algemeen duurder en minder effectief dan telefoon- en internetscreening. Internetscreening was iets kosteneffectiever dan telefoonscreening. De meest kosteneffectieve screeningsstrategie was internetscreening op een leeftijd van 50 jaar met herhaling na 5, 10, 15 en 20 jaar. Deze strategie kostte $€ 3699$ per voor kwaliteit gecorrigeerd levensjaar. Bij de drempel van $€ 20000$ per voor kwaliteit gecorrigeerd levensjaar was deze strategie met $100 \%$ zekerheid kosteneffectief ten opzichte van geen screening en met $69 \%$ zekerheid kosteneffectiever dan elke andere strategie.

Het screeningsinstrument dat gebruikt was in de kwalitatieve studie van Hoofdstuk 2 gaf de screeningsuitslag op drie manieren weer: een audiogram, een tekstboodschap, en een hoorleeftijd. De screendeelnemers zagen de hoorleeftijd als de meest waardevolle indicator van de ernst van hun gehoorverlies. Onze bevindingen suggereren dat het presenteren van de hoorleeftijd een zinvolle methode is voor mensen onder de 65 . Echter, een longitudinale kwantitatieve studie is nodig om te bepalen of een screeningsuitslag weergegeven als hoorleeftijd inderdaad effectiever is in het stimuleren van volwassenen om hulp te zoeken en te starten met rehabilitatie dan een andere weergave.

\section{Discussie}

Tenslotte worden in Hoofdstuk 7 de verschillende studies uit dit proefschrift bediscussieerd. Ook worden de onderzoeksvragen beantwoord en aanbevelingen gedaan voor toekomstig onderzoek. Geconcludeerd wordt dat internetscreening op een leeftijd van 50 jaar met herhaling na 5, 10, 15 en 20 jaar een kosteneffectieve, onschadelijke en toegankelijke screeningsstrategie is. Deze strategie lijkt geschikt te zijn voor nationale implementatie, maar we raden aan eerst een lokale pilot uit te voeren. Informatieverstrekking over alle mogelijke rehabilitatieopties en over naar wie en waar men toe kan voor elk type rehabilitatie dient onderdeel uit te maken van het screeningsprogramma. Onderzoek is nodig naar strategieën om te voorzien in de behoefte aan nazorg onder hoortoestelbezitters die in verzorgingshuizen wonen. Verder is onderzoek nodig naar de (kosten-)effectiviteit van hoortoesteltraining gericht op het vergroten van iemands 
zelfvertrouwen met betrekking tot succesvol hoortoestelgebruik. Andere belangrijke gebieden voor verder onderzoek zijn de kosteneffectiviteit van rehabilitatieopties anders dan hoortoestellen en de impact van hoorleeftijd op het zoeken van hulp voor slechthorendheid. 
About the author 



\section{Curriculum vitae}

Anouk Linssen was born on the $14^{\text {th }}$ of October in 1986 in Sittard, the Netherlands. She completed her secondary education at Connect College Echt in 2004. That same year she started studying Biomedical Sciences at the Radboud University in Nijmegen, with a specialisation in Epidemiology. After obtaining her Master's degree in 2009, she started working at the School for Mental Health and Neuroscience of the Faculty of Health, Medicine and Life Sciences of Maastricht University and the Department of Otorhinolaryngology, Head and Neck Surgery of the Maastricht University Medical Centre, where she carried out the PhD research that resulted in this thesis.

\section{Publications}

Linssen AM, van Boxtel MPJ, Joore MA, \& Anteunis LC. Predictors of hearing acuity: cross-sectional and longitudinal analysis. J Gerontol A Biol Sci Med Sci 2014;69(6):759765.

Linssen AM, Joore MA, Minten RKH, van Leeuwen YD, \& Anteunis LJC. Qualitative interviews on the beliefs and feelings of adults towards their ownership, but non-use of hearing aids. Int J Audiol 2013;52(10):670-677.

Linssen AM, Joore MA, Theunissen EJJM, \& Anteunis LJC. The effects and costs of a hearing screening and rehabilitation program in residential care homes for the elderly in the Netherlands. Am J Audiol 2013;22(1):186-189.

Linssen AM, Anteunis LJC, \& Joore MA. The cost-effectiveness of different hearing screening strategies for 50- to 70-year-old adults: a Markov model. Submitted. 



\section{Dankwoord}



Het is gelukt! Na ruim vier jaar ploeteren is mijn proefschrift eindelijk af. Nu is het moment daar om de mensen te bedanken die dit proefschrift mede mogelijk hebben gemaakt, met hun investeringen - in welke vorm dan ook - in dit boekje of in mij.

Allereerst wil ik mijn copromotoren Lucien Anteunis en Manuela Joore bedanken. Lucien, omdat we op dezelfde afdeling zaten heb ik met jou het meeste contact gehad. Ik vond het erg fijn dat je deur altijd voor me open stond. Als ik te snel vooruit wilde gaan en belangrijke zaken uit het oog dreigde te verliezen was jij er om me terug te fluiten of bij te sturen. Dit, en jouw uiterst zorgvuldige controle op het correct gebruik van komma's, punten en hoofdletters, heeft de kwaliteit van mijn artikelen naar een hoger niveau getild. Manuela, als ik te lang stil bleef staan en verstrikt dreigde te raken in details was jij er om me aan te zwengelen. Je frisse ideeën en besluitvaardigheid hebben mij enorm vooruit geholpen. Prof. dr. Kremer, beste Bernd, bedankt dat je mijn promotor wilt zijn.

De leden van de beoordelingscommissie, te weten voorzitter prof. dr. R.J. Stokroos, prof. dr. G.J. Dinant, prof. dr. S.M.A.A. Evers, dr. J.P.C. Grutters en prof. dr. ir. A.F.M. Snik, wil ik van harte bedanken voor het beoordelen van dit proefschrift.

De interviewdeelnemers bedank ik voor hun medewerking en tijd. Ook dank aan Ruud Backerra van Beter Horen en alle anderen die geholpen hebben bij de werving van geschikte interviewkandidaten. Yvonne van Leeuwen, jouw enthousiasme heeft me verleid tot het doen van kwalitatief onderzoek. Ik ben je erg dankbaar voor die leerzame ervaring, voor de prettige samenwerking en voor het feit dat je Rianne Minten hebt weten te strikken om mij als stagiaire te assisteren bij mijn onderzoek. Rianne, bedankt voor je hulp en gezelligheid. Martin van Boxtel, jij stond altijd voor me klaar als ik weer eens vragen had over de MAAS data of nood had aan een gedachtewisseling over het plan van aanpak. Dankjewel! Verder bedank ik Eric Theunissen en de medewerkers van Adelante Audiologie \& Communicatie voor het mogelijk maken van Hoofdstuk 4. Ook dank aan iedereen die input geleverd heeft voor het kosteneffectiviteitsmodel.

Mijn collega's van het Audiologisch Centrum wil ik bedanken voor hun betrokkenheid en voor de gezelligheid tijdens lunches en dagjes uit. In het bijzonder dank aan de audiologie-assistenten die meegeholpen hebben met het verzamelen van gegevens die ik nodig had voor het valideren van de Siemens HearCheck Navigator. Mickey, het was fijn om zo'n meelevende en behulpzame kamergenoot als jou te hebben. Bedankt dat je altijd zo met mij begaan was. Elke, wat was ik blij toen jij in het azM kwam werken. Een fulltime collega-promovenda om lief en leed mee te delen! En wat voor een! Bovendien superhandig om een audioloog op de kamer te hebben bij het schrijven van een proefschrift op audiologisch gebied. Elke, Remo, Marc en Jasper, bedankt voor de ontspannende koffiepauzes en verlichtende onderzoekerslunches. Ook zonder loungebank vormden jullie een geweldig klankbord.

Lieve ouders, schoonouders en vrienden, bedankt dat jullie er voor me waren wanneer ik behoefte had aan een luisterend oor, een advies, een gezonde maaltijd, een iets minder gezonde barbecue, een glas wijn, een biertje, een interessante nieuwtje, een 
sappige roddel, of een gezellig dagje uit. Laura, samen trokken we 10 jaar geleden van de middelbare school in Echt naar de universiteit in Nijmegen om aan de opleiding Biomedische Wetenschappen te beginnen. Ik vind het fijn dat je naast me wilt staan op de dag dat ook ik eindelijk mijn studententijd officieel achter me laat.

Tim, wir haben es geschafft! Vielen Dank für alles! 